\title{
Competitive Insurance Markets with Unbounded Cost
}

\author{
Yehuda John Levy* \& André Veiga ${ }^{\dagger}$
}

This version: January 10, 2021

\begin{abstract}
Azevedo and Gottlieb [2017] (AG) define a notion of equilibrium that always exists in the Rothschild and Stiglitz [1976] (RS) model of competitive insurance markets, provided costs are bounded. However, equilibrium predictions are fragile: introducing an infinitesimal mass of high-cost individuals discretely increases all prices and reduces coverage for all individuals. We study sensitivity w.r.t cost bounds by considering sequences of economies with increasing upper bounds of cost, and determining whether their equilibria converge. We present sufficient conditions under which AG equilibrium exists when cost is unbounded. For simple insurance markets, we derive a necessary and sufficient condition for existence: surplus from insurance increases faster than linearly with expected cost. This condition is empirically common. If the condition fails, a higher bound on cost results in market unraveling: all prices diverge and, in the limit, an AG equilibrium does not exist. We use these results to show that the equilibrium for an insurance market with an unbounded continuum of types is characterized by a simple differential equation. We also provide examples of non-existence for a (single-product) market for lemons with unbounded cost.
\end{abstract}

JEL: C62, G22, I13, D82

Keywords: Adverse Selection, Asymmetric Information, Insurance, Equilibrium Existence

*Adam Smith Business School, University of Glasgow, Glasgow, UK; john.levy@glasgow.ac.uk.

†Imperial College Business School, South Kensington Campus, London, UK; a.veiga@imperial.ac.uk. 


\section{Introduction}

The Rothschild and Stiglitz [1976, henceforth RS] model of competitive insurance markets has (at least) two limitations. First, there need not exist a pure-strategies Nash equilibrium. Second, equilibrium is sensitive to assumptions about the upper bound of the cost distribution: introducing an infinitesimal mass of high cost individuals discretely increases the equilibrium price of all contracts (Mailath et al. [1993]). Azevedo and Gottlieb [2017, henceforth AG] suggest a notion of equilibrium that always exists in the RS context. By tackling existence, the AG equilibrium concept allows us to focus on the second limitation, sensitivity to cost bounds. In fact, AG equilibrium is only guaranteed to exist when cost is bounded. Moreover, AG equilibrium predictions are similarly sensitive to cost bounds. This limits the policy usefulness of insurance models because it is often unclear what is a reasonable assumption for the upper bound of cost. Moreover, it is unclear what economic environments feature the sensitivity of the RS setting.

This article derives conditions under which screening markets with adverse selection (and, in particular, insurance markets) have AG equilibria which are robust w.r.t. cost bounds. We do so by considering sequences of truncated economies where cost is bounded, and progressively relaxing this truncation. Our measure of robustness w.r.t. cost bounds is whether the equilibria of the truncated economies converge. That is, whether an equilibrium exists for the limit economy with unbounded cost.

Our motivation is not that unbounded costs are particularly relevant or realistic. Instead, we take existence of equilibria as our measure of whether a model's predictions are sensitive to assumptions about the support of costs. If equilibria of bounded economies converge to an equilibrium of the unbounded economy, then model predictions are robust w.r.t. assumptions on cost bounds. Conversely, if assumptions about the support of cost can have an unbounded impact on equilibrium predictions, this can be diagnosed by determining that the limit economy has no equilibrium.

We first consider the setting described in AG. Intuitively, an AG equilibrium is a set of prices and choices such that: a) individuals optimize; b) each contract breaks even; and c) choices and prices of non-traded contracts are robust to small perturbations in fundamentals. In this general setting, we provide sufficient conditions for equilibrium existence when cost is unbounded. However, our most general existence result has a limitation: it does not impose conditions directly on model primitives.

We then focus on the case of insurance markets, as in RS. We allow individuals to differ in risk and risk aversion (but a single parameter determines both, so types are effectively one-dimensional), and assume costlier types have higher marginal willingness to pay for insurance generosity.

In the two-type RS model, there exists a unique AG equilibrium which predicts the 
same allocation as the pure strategies Nash equilibrium (when it exists). We provide a novel characterization of the (unique) AG equilibrium for an arbitrary number of (possibly unbounded) discrete or continuous types. We then show that, if cost is unbounded, the price of full insurance is also unbounded in any equilibrium.

Our main contribution is a condition on model primitives which is both necessary and sufficient for existence of equilibrium when cost is unbounded. Intuitively, this condition requires that surplus from insurance increases faster than linearly with expected cost. The condition divides insurance economies into "robust" and "fragile" w.r.t. cost bounds. For fragile economies, as cost becomes unbounded, the market unravels: the price of each alternative increases without bound and the chosen level of coverage of each type converges to zero. For fragile economies with unbounded cost, an equilibrium does not exist. Conversely, economies that are robust w.r.t. cost bounds have an equilibrium no matter what assumption is imposed on the support of cost.

This condition is intuitive and empirically relevant. For instance, if utilities are CARA and wealth shocks Gaussian, equilibrium exists if the variance of wealth shocks increases (asymptotically) faster than linearly relative to the mean of these shocks. This condition is satisfied in the empirical findings of Handel et al. [2015] and, more broadly, it seems empirically common that individuals with higher expected cost experience higher variance in insurable shocks (Brown et al. [2014], Hendren [2013]) and therefore obtain higher surplus from insurance. Therefore, insurance economies robust w.r.t. cost bounds seem empirically common. The results further imply that, if individuals differ only in their cost (as in RS), and costs are unbounded, then the economy is fragile: AG equilibrium does not exist.

We use these results to characterize the equilibrium of an insurance market with an unbounded continuum of types, and show it is defined by a simple differential equation. We also characterize the equilibrium for an economy with unbounded discrete types.

We then briefly consider markets for lemons (Akerlof [1970], Einav et al. [2010]), where there is a single non-zero insurance contract available. Even in such simple settings, unbounded costs can result in equilibrium non-existence.

We extend several results to more general insurance markets. For instance, unbounded costs imply unbounded prices even in more general settings where types are truly multidimensional, so there is pooling of multiple types in each contract. This result identifies a large class of economies with unbounded costs where equilibrium does not exist.

Our results are useful in two ways. First, when model primitives are well known, we provide a novel characterization of equilibrium, showing under which conditions a wider distribution of cost types causes a market to unravel. Second, when model primitives are uncertain, we identify conditions under which assumptions about the support of the cost distribution have a large impact on equilibrium. 
Section 2 summarizes the setting and results of AG. Section 3 describes our general existence result. Section 4 specializes the model to insurance markets. Section 5 considers markets for lemons. Section 6 generalizes several results. Section 7 concludes. All proofs are in the Appendix (including the Online Appendix), although we provide some intuition for the proofs in the main text.

\section{A Summary of AG}

We now describe the model presented in AG and their existence result, generalized appropriately to encompass unbounded costs and other aspects we will require. A consumer type is a vector $\theta \in \Theta$, where $\Theta$ is a Polish space with measure $P .{ }^{1}$ The type $\theta$ can describe each individual's risk, risk aversion, wealth, etc. An alternative is a vector $x \in X$, where $X$ is a locally compact Polish space. Alternatives $x$ can be characterized by deductibles, co-insurance rates, etc. Price is $p \in \mathbb{R}_{+}$. A contract is a pair $(x, p) \in X \times \mathbb{R}_{+}$. We consider Borel-measurable price functions $p: X \rightarrow \mathbb{R}_{+}$where $p(x)$ is the price of alternative $x$. An economy $\mathcal{E}$ is a triple $\mathcal{E}=[\Theta, X, P]$.

Utility is a continuous function $u(\theta, x, p)=u_{\theta}(x, p)$, where $u: \Theta \times X \times \mathbb{R}_{+} \rightarrow \mathbb{R}$ is strictly decreasing in $p$. Cost is a continuous function $c(\theta, x) \geq 0$, where $c: \Theta \times X \rightarrow \mathbb{R}_{+}$. Cost depends on type $\theta$ which creates the possibility of adverse selection.

An allocation is a distribution $\alpha$ on $\Theta \times X$ with marginal $P$ on $\Theta$, and marginal $\alpha_{X}$ on $X$, such that $\int_{\Theta \times X} c(\theta, x) d \alpha<\infty$. Intuitively, $\alpha(\{\theta, x\})$ is the mass or density of types $\theta$ purchasing alternative $x$ under allocation $\alpha$.

AG assume that $\Theta, X$ are compact and $c(\theta, x)$ is continuous, which implies that cost is bounded. We will allow $\Theta$, and therefore cost, to be unbounded. We assume only that, for each compact sub-set of alternatives $K \subseteq X$, if all agents choose alternatives in $K$, expected cost is finite: $\int_{\Theta} \sup _{x \in K} c(x, \theta) d P(\theta)<\infty$.

AG define a "weak equilibrium" as a price function and an allocation $(p, \alpha)$ where individuals are maximizing utility and each contract breaks even.

Definition 1. A pair $(p, \alpha)$ constitute a weak equilibrium of an economy $\mathcal{E}=[\Theta, X, P]$ if, for $\alpha$-a.e. $(\theta, x) \in \Theta \times X$ the following conditions hold. First, individuals maximize: for $\alpha$-a.e. $(\theta, x)$, we have $\sup _{x^{\prime} \in X} u_{\theta}\left(p\left(x^{\prime}\right), x^{\prime}\right)=u_{\theta}(p(x), x)$. Second, each contract breaks even: for $\alpha$-a.e. $x$, we have $p(x)=\mathbb{E}_{\alpha}[c \mid x] .^{2}$

Typically, there exists many weak equilibria because, if $x$ is not traded, $p(x)$ is arbitrary. This motivates AG's definition of equilibrium: a weak equilibrium that is robust

\footnotetext{
${ }^{1} \mathrm{~A}$ Polish space is a complete, separable metrizable space.

${ }^{2}$ The conditional expectation is well-defined, since $X$ is locally compact and, for each compact set $K \subseteq X, \int_{\Theta} \sup _{x \in K} c(x, \theta) d P(\theta)<\infty$.
} 
to the introduction of a small mass of zero-cost "behavioral" consumers who purchase every alternative $x \in X .^{3}$ More precisely, an equilibrium is the limit of a sequence of weak equilibria of perturbed economies $\mathcal{E}_{j}$ where the mass of behavioral types vanishes as $j \rightarrow \infty$. We use "equilibrium" exclusively to refer to the equilibrium notion of AG (formalized below after some generalization).

Definition 2. Consider the economy $\mathcal{E}=[\Theta, X, P]$, and the sequence of perturbed economies $\mathcal{E}_{j}=\left[\Theta \cup \bar{X}_{j}, \bar{X}_{j}, P+\eta_{j}\right]$. Let $\left(\bar{X}_{j}\right)_{j \in N}$ be a sequence of finite subsets of $\bar{X}$ which converge to $\bar{X}$ in the sense of Haussdorf, ${ }^{4}$ where $\bar{X}$ is a Polish space such that $X$ is dense in $\bar{X}{ }^{5}$ Let $\left(\eta_{j}\right)_{j \in N}$ be a sequence of measures, with $\eta_{j}$ supported on $\bar{X}_{j}$, strictly positive on $\bar{X}_{j}$, and $\eta_{j}\left(\bar{X}_{j}\right) \rightarrow 0$. Suppose there exists a sequence of pairs $\left(p_{j}, \alpha_{j}\right)_{j \in \mathbb{N}}$, satisfying the following conditions. First, $\left(p_{j}, \alpha_{j}\right)$ is a weak equilibrium of $\mathcal{E}_{j}$, where the behavioral type $x \in \bar{X}_{j}$ has zero cost and prefers $x$ to any other alternative regardless of price. Second, $\alpha_{j} \rightarrow \alpha$ weakly. ${ }^{6}$ Third, whenever $\left(x_{j}\right)_{j \in \mathbb{N}}$ converges to $x \in X$ with $x_{j} \in \bar{X}_{j}$, then $p_{j}\left(x_{j}\right) \rightarrow p(x)$. Then, the pair $(p, \alpha)$ is an equilibrium of $\mathcal{E}=[\Theta, X, P]$.

AG prove every economy has an equilibrium (Theorem 1) if certain technical conditions hold. Importantly, AG require $\Theta, X$ be compact.

Theorem 1. Suppose that $X, \Theta$ are compact metric spaces. Suppose also that u obeys a form of Lipschitz-ness in $X$ uniformly over types, ${ }^{7}$ and thatc is continuous (which implies $c$ is bounded). Then, an equilibrium exists.

AG derive additional equilibrium properties (their Proposition 1). An equilibrium is a weak equilibrium so, in equilibrium, a.e. agent is optimizing (we use this implicitly throughout this article). The price function $p(\cdot)$ is Lipschitz and continuous. Every alternative not traded in equilibrium has a price low enough that some individual is indifferent between buying it and not, and the cost of that individual at the non-traded alternative is at least as high as the price of the alternative. The continuity of $p(\cdot)$ and the fact that equilibrium is a weak equilibrium is generalized to environments with unbounded types in Section H of the Online Appendix.

\footnotetext{
${ }^{3} \mathrm{~A}$ similar construction is used by Dubey and Geanakoplos [2002]. AG prove that every equilibrium, under their compactness and other assumptions, is a weak equilibrium.

${ }^{4}$ I.e., for each $x \in \bar{X}$, there is $\left(x_{n}\right)_{n \in \mathbb{N}}$ converging to $x$ with $x_{n} \in \bar{X}^{n}$ for each $n \in \mathbb{N}$.

${ }^{5}$ Formally, $X$ embeds to a dense subset of $\bar{X}$. We disregard such technicalities for brevity at no cost to the generality. In insurance markets, we often take $X=[0,1)$ which naturally embeds in $\bar{X}=[0,1]$.

${ }^{6}$ That is, for each $f: \Theta \times X \rightarrow \mathbb{R}$ continuous and bounded, we have $\int f d \alpha^{n} \rightarrow \int f d \alpha$.

${ }^{7}$ Formally, there exists $L$, such that for any $p \leq p^{\prime}$ in the image of $c$, any $x, x^{\prime} \in X$, and any type $\theta \in \Theta$, if $u(x, p, \theta) \leq u\left(x^{\prime}, p^{\prime}, \theta\right)$, then $p^{\prime}-p \leq L d\left(x, x^{\prime}\right)$, where $d(\cdot, \cdot)$ is a metric. This is Assumption 2 in AG. If utility is of the form $u(\theta, x, p)=v(\theta, x)-p$, this amounts to $u(\theta, x, p)$ being Lipschitz in $x$, uniformly in $\theta$ (same Lipschitz constant for all $\theta$ ).
} 


\section{Existence with Unbounded Cost}

We now describe conditions under which equilibrium exists when cost is unbounded (Proposition 1). This result has a limitation: it imposes conditions on endogenous objects. In Section 4, we provide conditions on primitives that are necessary and sufficient for Proposition 1 to hold. However, Proposition 1 is a crucial building block of our analysis of insurance markets in Section 4, so we present it here briefly.

We consider sequences of bounded economies, approximating an economy with unbounded cost. We then consider whether their equilibria converge. The truncated economy $\mathcal{E}^{n}=\left[\Theta^{n}, \bar{X}, P\left(\cdot \mid \Theta^{n}\right)\right]$ has a bounded type space $\Theta^{n}$. We consider a sequence of compact subsets $\Theta^{1} \subseteq \Theta^{2} \subseteq \cdots \subseteq \Theta$ with $\cup_{n} \Theta^{n}=\Theta$. We assume that for each $n \in \mathbb{N}$, each $\theta \in \Theta^{n}$ and each $\theta^{\prime} \in \Theta \backslash \Theta_{n}$, we have $c(\theta, \cdot) \leq c\left(\theta^{\prime}, \cdot\right)$. That is, types not in $\Theta^{n}$ have costs higher than those in $\Theta^{n}$. The alternative space $\bar{X}$ is the compactification of $X$ (the union of $X$ with its limit points). We assume that $c(\cdot, \cdot)$ and $u(\cdot, \cdot, \cdot)$ extend continuously to $\bar{X} \times \Theta$ and $\bar{X} \times \Theta \times \mathbb{R}_{+}$. Conditional distributions are standard: $P\left(\cdot \mid \Theta^{n}\right)=P\left(\cdot \cap \Theta^{n}\right) / P\left(\Theta^{n}\right)$. By Theorem 1 , each $\mathcal{E}^{n}$ has an equilibrium $\left(p^{n}, \alpha^{n}\right)$. Our first existence result will use the following condition.

Condition 1. There exists a cost $c_{0}>0$ such that, for large enough $k$ and $n>k$, types in $\Theta^{n} \backslash \Theta^{k}$ purchase only options $x$ with cost $p^{n}(x) \geq c_{0}$. Formally: $\alpha^{n}\left(\left\{p^{n}(x) \geq c_{0}\right\} \mid \alpha \in\right.$ $\left.\Theta_{n} \backslash \Theta_{k}\right)=1$.

Condition 1 requires that costly types do not purchase cheap contracts. This condition holds naturally in many models, including those of Section $4 .^{8}$

Proposition 1. For each $n \in \mathbb{N}$, let $\left(p^{n}, \alpha^{n}\right)$ be an equilibrium of the truncated economy $\mathcal{E}^{n}=\left[\Theta^{n}, \bar{X}, P\left(\cdot \mid \Theta^{n}\right)\right]$, and suppose that the following assumptions hold. First, there is a function $p: X \rightarrow \mathbb{R}_{+}$s.t. $p^{n} \rightarrow p$ uniformly on compact subsets of $X$. Second, there is a distribution $\alpha$ on $\Theta \times X \subseteq \Theta \times \bar{X}$ s.t. $\alpha^{n} \rightarrow \alpha$ weakly. Third, Condition (1) holds. Then, $(p, \alpha)$ is an AG equilibrium of the unbounded economy $\mathcal{E}=[\Theta, X, P]$.

Proof. The proof uses a diagonalization argument. Each $\mathcal{E}^{n}$ has an equilibrium $\left(p^{n}, \alpha^{n}\right)$ which is the limit of the weak equilibria $\left(p_{j}^{n}, \alpha_{j}^{n}\right)$ of a sequence of perturbed economies $\mathcal{E}_{j}^{n}$ which have a vanishing mass of behavioral types, as in AG. We then consider the sequence $\mathcal{E}^{n}$ and show that an appropriate diagonal of weak equilibria $\left(p_{j_{n}}^{n}, \alpha_{j_{n}}^{n}\right)$ converge to an AG equilibrium of $\mathcal{E}$ when $n \rightarrow \infty$. Finally, we modify the equilibria on this diagonal to include all types, as $\alpha_{j}^{n}$ only allocates types in $\Theta^{n}$. Due to the behavioral types, this can be done without changing the price $p_{j}^{n}$. See Appendix A.1 for details.

\footnotetext{
${ }^{8}$ Indeed, for insurance markets, in some neighborhood of full insurance $(x=1)$, the equilibrium price $p(\cdot)$ (and hence $p^{n}$ ) satisfy $p^{n}(x) \geq c_{0}$ and $p(x) \geq c_{0}$. Moreover, types with high riskiness (and hence high willingness to pay) will purchase coverage in this neighborhood; this intuition is formalized in Lemma 1 of Section A.
} 


\section{Simple Insurance Markets}

This section contains the bulk of our contribution. We specialize the model of Section 2 to insurance settings. First, we provide a novel characterization of the (unique) equilibrium with arbitrarily many (and possibly unbounded) types. Second, we derive a condition on model primitives that is necessary and sufficient for existence in insurance markets with unbounded cost. Third, we characterize equilibrium for insurance economies with unbounded continuous types and unbounded discrete types.

\subsection{A Model of Insurance}

An individual of type $\theta \in \Theta$ is exposed to a stochastic wealth loss described by the random variable $Z_{\theta}$. Let $\mu: \Theta \rightarrow \mathbb{R}_{++}$be a continuous map assigning type $\theta$ to her expected loss. Let risk types be $\mu_{\theta}=\mu(\theta)=\mathbb{E}\left[Z_{\theta}\right]$. We assume $\mu_{\theta}>0 P$-a.s. Importantly, we allow the marginal distribution of $\mu_{\theta}$ to not be bounded: $\forall M \geq 0, P\left(\left\{\theta \mid \mu_{\theta} \geq M\right\}\right)>0$. Denote the marginal probability of $\mu$ by $P_{\mu}=P \circ \mu^{-1}$, and let $\underline{\mu}=\min \left(\operatorname{supp}\left(P_{\mu}\right)\right)$, where supp denotes the support.

We assume the following parameterization of alternatives $x \in X$. An individual who purchases alternative $x \in[0,1]$ is only exposed to the random shock $(1-x) Z_{\theta}$, with the remaining $x Z_{\theta}$ being absorbed by the insurer. Full insurance corresponds to $x=1$, and zero insurance to $x=0$. The alternative space, unless otherwise specified, is either $X=[0,1]$ or $X=[0,1)$. We may choose to use $X=[0,1)$, because, as we will show in Section 6.5, any equilibrium with unbounded cost must have $\lim _{x \rightarrow 1} p(x)=\infty .^{9}$

The cost to a risk neutral insurer of alternative $x$ sold to type $\theta$ is $c(\theta, x)=x \mu_{\theta}$. We assume that even if each individual chooses full insurance $(x=1)$, expected cost is finite: $\int_{\Theta} \mu_{\theta} d P<\infty$.

We make the following assumption regarding utilities.

Assumption 1. Utility (certainty equivalent) is $u(\theta, x, p)=x \mu_{\theta}+g(x) \nu_{\theta}-p$, for some continuous function $\nu_{\theta}: \Theta \rightarrow \mathbb{R}_{++}$. We assume $g:[0,1] \rightarrow \mathbb{R}$ is twice continuously differentiable, with $g^{\prime}>0$ in $x \in[0,1), g^{\prime}(1)=0, g^{\prime \prime} \leq 0$, and $g^{\prime \prime}(1)<0$.

Utility from alternative $x$ has three components. First, $x \mu_{\theta}$ is the individual's expected cost, passed on to the insurer. Second, $g(x) \nu_{\theta}$ captures the individual's surplus from insurance, where $\nu_{\theta}$ is the individual's "insurance value." Notice that $g(x)$ is common to all individuals, while $\nu_{\theta}$ is heterogeneous. If $\nu_{\theta}>0$, individuals are willing to pay for insurance above their expected cost (for instance, due to risk aversion). There are decreasing marginal returns from insurance. At full insurance, insurance has zero

\footnotetext{
${ }^{9}$ In fact, all the results in Section 4 hold, with natural minor modifications, if the upper bound of coverage is replaced with $x_{\max } \in(0,1)$.
} 
marginal value: $g^{\prime}(1)=0$. We assume certainty equivalents are quasilinear in prices. This assumption is not innocuous, but is common in models with constant absolute risk aversion (CARA). ${ }^{10}$ These assumptions imply that types are effectively two-dimensional, since only $\left(\mu_{\theta}, \nu_{\theta}\right)$ matter for decisions and costs.

The marginal willingness to pay for additional insurance is

$$
w_{\theta}(x)=\frac{\partial u_{\theta}}{\partial x}(x, p)=\mu_{\theta}+g^{\prime}(x) \nu_{\theta} \geq \mu_{\theta} .
$$

with equality iff $x=1$. By Assumption 1, $w_{\theta}$ is independent of price.

We also make the following assumptions on the distribution of types.

Assumption 2. $\nu_{\theta}=\nu\left(\mu_{\theta}\right)$ with $\nu(\cdot)$ a weakly increasing function, locally Lipschitz and $\nu\left(\mu_{\theta}\right)>0$ for $\mu_{\theta}>\mu$.

Assumption 3. $P_{\mu}$ is absolutely continuous with a.e. positive density on a (bounded or unbounded) interval, or purely atomic with finitely many atoms in each bounded interval.

Assumption 2 implies the marginal willingness to pay $w_{\theta}$ is strictly increasing in cost $\mu_{\theta}$. This monotonicity is assumed in RS, Riley [1979], AG's discussion of the RS and Akerlof [1970] models, and in all models of insurance with one-dimensional types we are aware of. ${ }^{11}$ Under Assumption 2, two types with the same risk also have the same marginal willingness to pay, so types are effectively one-dimensional: $\left(\mu_{\theta}, \nu\left(\mu_{\theta}\right)\right) .{ }^{12}$ Still, this setting generalizes RS (and AG's discussion of RS), where $\nu\left(\mu_{\theta}\right)$ was assumed constant. Assumption 2 also implies the Spence [1973]-Mirrlees [1971] single-crossing condition: indifference curves of any two agents intersect at most once. ${ }^{13}$

Assumption 3 imposes regularity on the marginal distribution of $\mu_{\theta}$. The condition is technical, mild, and satisfied in all insurance models that we are aware of.

Example 1 describes a setting where Assumptions 1 and 2 hold. This CARA-Gaussian parameterization is used, for instance, in Veiga and Weyl [2016], Levy and Veiga [2017].

Example 1. Suppose wealth shocks are Gaussian: $Z_{\theta} \sim \mathcal{N}\left(\mu_{\theta}, \sigma_{\theta}^{2}\right)$. Suppose utility is CARA: $U_{\theta}(y)=e^{-a_{\theta} y}$ where $y$ is wealth and $a_{\theta}$ is risk aversion. Each type has initial wealth $w_{\theta}$. Then, insurance value is $\nu_{\theta}=a_{\theta} \sigma_{\theta}^{2}$ and certainty equivalents are $u(\theta, x, p)=x \mu_{\theta}+$ $\frac{1}{2}\left(1-(1-x)^{2}\right) \nu_{\theta}-p$. Marginal willingness to pay for insurance is $w_{\theta}(x)=\mu_{\theta}+(1-x) \nu_{\theta}$

\footnotetext{
${ }^{10}$ Notice that quasi-linearity implies that utility is 1 -Lipschitz in price.

${ }^{11}$ This assumption is not present in models with multidimensional types. Villeneuve [2003] and Smart [2000] consider heterogeneity in risk and risk aversion, but costs are bounded. Wambach [2000], Crocker and Snow [2011], De Meza and Webb [2001] and Snow [2009] consider other forms of multidimensional heterogeneity, again with bounded cost.

${ }^{12}$ For this reason, we sometimes write, for instance, $w_{\mu}$ instead of $w_{\theta}$.

${ }^{13}$ In Section 6.2 we discuss a relaxation of this assumption, which is equivalent to single-crossing under mild regularity conditions.
} 
AG equilibrium with 2 types

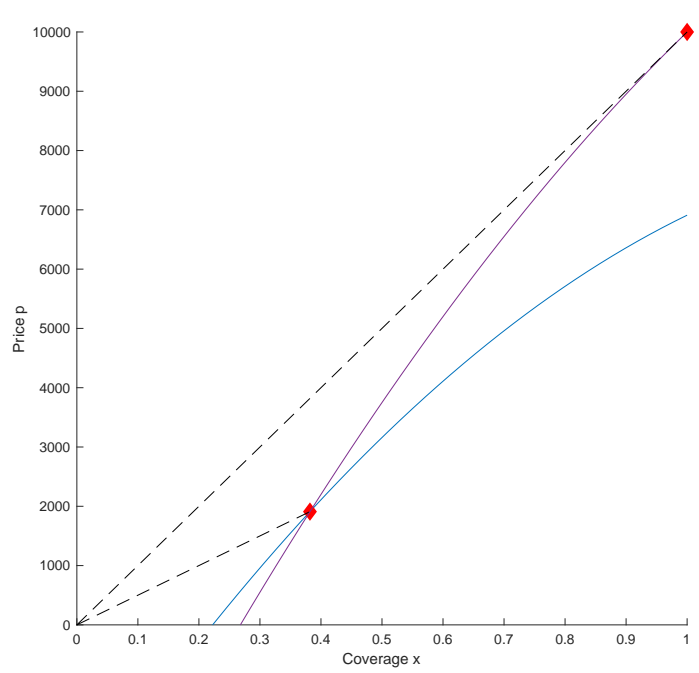

Figure 1: Equilibrium in an insurance market with 2 types. Dashed black lines represent zero-profit lines for each type. Solid lines represent each type's indifference curves at her chosen contract. The equilibrium price $p(x)$ is the upper envelope of the two indifference curves, and the line $p=0$. Red diamonds represent the contracts $(p, x)$ chosen by each type.

and Assumption 1 is satisfied. Assumption 2 is satisfied if $\nu_{\theta}=\nu\left(\mu_{\theta}\right)$ for any weakly increasing $\nu(\cdot)$.

AG specialize their general model to insurance markets with two cost types, as in RS. In insurance markets, since $c(\theta, x)=x \mu_{\theta}$, the break-even condition becomes

$$
p(x)=x \cdot \mathbb{E}_{\alpha}\left[\mu_{\theta} \mid x\right], \quad \alpha \text {-a.e. } x .
$$

AG derive several properties of equilibrium for insurance markets (AG's Corollary 1). An equilibrium exists and is unique. The high-cost type obtains full insurance $(x=1)$, and is indifferent between her choice and the contract chosen by the low-cost type. ${ }^{14}$ The equilibrium price function $p(x)$ is the upper envelope of $p=0$ and each type's indifference curve at her chosen contract. Notice that only 2 contracts are purchased in equilibrium but $p(x)$ is defined for all $x \in X$. This is illustrated in Figure 1.

\subsection{Equilibrium Characterization}

We generalize AG by characterizing equilibrium in insurance markets with arbitrarily many (possibly unbounded) types. Recall that $X=[0,1]$ when cost is bounded and

\footnotetext{
${ }^{14}$ In the RS model, the AG equilibrium has the same allocation as the Riley [1979] equilibrium, which is also the Nash equilibrium, when the latter exists. However, a Riley [1979] equilibrium need not always exist, as shown by Azevedo and Gottlieb [2016].
} 
$X=[0,1)$ otherwise.

Theorem 2. Suppose Assumptions 1, 2 and 3 hold for a bounded or unbounded insurance economy $\mathcal{E}=[\Theta, X, P]$. There is at most one equilibrium $(p, \alpha)$ for $\mathcal{E}$. If an equilibrium exists, it has the following properties.

1. Price $p(x)$ is continuous, and it is strictly increasing in $\{x \mid p(x)>0\}$.

2. There is a continuous and strictly increasing mapping $\sigma: \operatorname{supp}\left(P_{\mu}\right) \rightarrow X$ that assigns to each type $\mu_{\theta}$, the alternative $\sigma\left(\mu_{\theta}\right)$ that she chooses $\alpha$-a.s.

3. Each contract breaks even: $P-$ a.s., $p\left(\sigma\left(\mu_{\theta}\right)\right)=\mu_{\theta} \cdot \sigma\left(\mu_{\theta}\right)$.

4. Full insurance $(x=1)$ is in the support of the equilibrium and zero insurance $(x=$ 0) is purchased by a set of individuals with measure zero.

5. Price is Lipshitz in any interval bounded away from full insurance $(x=1)$; if $\mu_{\theta}$ is bounded P-a.s., price is Lipshitz.

6. If $P_{\mu}$ is discrete, each type is indifferent between the contract she chooses $\alpha$-a.s. and the next highest coverage purchased in $\alpha .^{15}$

Proof. Except for uniqueness, Theorem 2 follows from the more general Proposition 8, proved in Appendix C. Uniqueness (if equilibrium exists) follows from Corollaries 2 and Corollary 4 for continuous types. For discrete types, uniqueness follows from Proposition 2 and Proposition $4 .^{16}$

When $\mu_{\theta}$ is essentially bounded, existence follows from Theorem 1, but Theorem 2 establishes uniqueness. ${ }^{17}$ When $\mu_{\theta}$ is unbounded, Theorem 2 shows equilibrium is unique if it exists. In equilibrium higher cost types purchase more generous insurance, full insurance is purchased by some type in equilibrium, and incentive compatibility binds "downwards." Theorem 2 also implies that, for unbounded economies, the equilibrium prices of the most generous contracts are unbounded.

Corollary 1. Under Assumptions 1, 2 and 3, if $P_{\mu}$ is not compactly supported, then in any equilibrium $\lim _{x \rightarrow 1} p(x)=\infty$.

\footnotetext{
${ }^{15}$ Formally: Let $\mu_{1}<\mu_{2}$ be two atoms of $P_{\mu}$ with no atom between them. Suppose type $\mu_{1}$ purchases $x_{1}$ and type $\mu_{2}$ purchases $x_{2} \alpha$-a.s.. Then, type $\mu_{2}$ is indifferent between $\left(x_{2}, p\left(x_{2}\right)\right)$ and $\left(x_{1}, p\left(x_{1}\right)\right)$.

${ }^{16}$ Although Theorem 2 is used to prove Corollary 2, Corollary 4, Proposition 2 and Proposition 4, these proofs do not rely on the uniqueness of equilibrium. Instead, those proofs rely only on the other properties listed in Theorem 2 (so the reasoning is not circular). We present the uniqueness as part of Theorem 2 for expositional simplicity, instead of leaving this conclusion for a later corollary.

${ }^{17}$ Recall that a random variable $\mu$ is essentially bounded if there exists some $M<\infty$ such that $\operatorname{Prob}(|\mu|>M)=0$.
} 
Proof. The allocation $\sigma\left(\mu_{\theta}\right)$ is strictly increasing, and prices are actuarily fair (Theorem 2). Hence, if cost is unbounded, the price of full insurance diverges.

We do not take Corollary 1 to predict infinite prices in any insurance market. Our goal in studying the equilibrium of unbounded economies is to determine robustness w.r.t. cost bounds. Corollary 1 will play a key role in our analysis by allowing us to easily show non-existence of equilibrium. Corollary 1 is also why, for economies with unbounded cost, we consider the (non-compact) alternative space $X=[0,1)$.

\subsection{Continuum of Types}

We now consider an insurance market with a continuum of cost types. We show that equilibrium (when it exists) is characterized by a simple differential equation. Riley [1979] showed that a Nash equilibrium (in pure strategies) does not exist. An AG equilibrium exists provided that costs are bounded (Theorem 1). Theorem 2 then shows that equilibrium (if it exists) is unique. Building on these, we derive a simple, necessary and sufficient condition for existence when cost is unbounded. When this condition fails, the economy is "fragile": assumptions about the support of costs can have large effects on equilibrium prices. For fragile economies, expanding the support of costs may result in unravelling: the equilibrium allocation to each type converges to zero. ${ }^{18}$

We consider an unbounded economy $\mathcal{E}=[\Theta, X, P]$ with alternative space $X=[0,1)$ (recall Corollary 1), cost types $\mu_{\theta} \in \Theta=[\underline{\mu}, \infty)$ and distribution $P$ with Lebesgue-a.e. positive density (Assumption 3).

We will consider a sequence of bounded economies $\mathcal{E}^{n}=\left[\Theta^{n}, \bar{X}, P\left(\cdot \mid \Theta^{n}\right)\right]$ which approximate $\mathcal{E}$, as in Section 3. Economy $\mathcal{E}^{n}$ has alternative space $\bar{X}=[0,1]$, cost types $\mu_{\theta} \in \Theta^{n}=\left[\underline{\mu}, \overline{\mu_{n}}\right]$ and distribution $P\left(\cdot \mid \Theta^{n}\right)=P\left(\cdot \cap \Theta^{n}\right) / P\left(\Theta^{n}\right)$. First, we characterize the equilibrium of $\mathcal{E}^{n}$. Building on this, we derive a necessary condition for equilibrium existence in $\mathcal{E}$. Finally, we show that this condition is also sufficient.

Fix some $n \in \mathbb{N}$. Since $\bar{X}, \Theta^{n}$ are compact, each $\mathcal{E}^{n}$ has an equilibrium $\left(p^{n}, \alpha^{n}\right)$ with a continuous increasing allocation rule $\sigma^{n}:\left[\underline{\mu}, \bar{\mu}_{n}\right] \rightarrow[0,1]$. We omit superscript $n$ for notational simplicity. For each type $\mu_{\theta} \in\left(\underline{\mu}, \overline{\mu_{n}}\right)$, the optimal choice is characterized, assuming price is differentiable, by the First Order Condition

$$
\left.\frac{\partial u_{\theta}}{\partial x}\right|_{x=\sigma\left(\mu_{\theta}\right)}=\mu_{\theta}+g^{\prime}\left(\sigma\left(\mu_{\theta}\right)\right) v\left(\mu_{\theta}\right)-p^{\prime}\left(\sigma\left(\mu_{\theta}\right)\right)=0 .
$$

(Appendix E considers the case where $p$ is not differentiable). Since $\sigma\left(\mu_{\theta}\right)$ is strictly increasing, it admits an inverse $\tau=\sigma^{-1}$, which implies

\footnotetext{
${ }^{18}$ This article is concerned only with robustness w.r.t. cost bounds. This is a different notion of robustness than, for instance, the one in Debreu [1970] where, informally, a robust economy is one where equilibria are locally unique and change continuously with fundamentals.
} 


$$
\tau(x)+g^{\prime}(x) v(\tau(x))-p^{\prime}(x)=0 .
$$

For every $x$ in the interior of the support of $\alpha_{X}$, contract $x$ breaks even:

$$
p(x)=\tau(x) \cdot x \Rightarrow p^{\prime}(x)=\tau(x)+x \cdot \tau^{\prime}(x) .
$$

Summing these implies

$$
\frac{\tau^{\prime}(x)}{\nu(\tau(x))}=\frac{g^{\prime}(x)}{x} .
$$

Then $\sigma^{n}\left(\mu_{\theta}\right)$ can be recovered by integrating both sides of (2) over $x$ and using the change of variables $\tau(x)=\mu_{\theta}$. Proposition 8 provides the boundary condition $\sigma^{n}(\bar{\mu})=1$. We therefore obtain the following result.

Corollary 2. The bounded economy $\mathcal{E}^{n}$ has a unique equilibrium $\left(p^{n}, \alpha^{n}\right)$, where the choice rule $\sigma^{n}\left(\mu_{\theta}\right)$ satisfies, $\forall \mu_{\theta} \in\left[\underline{\mu}, \overline{\mu_{n}}\right]$,

$$
\int_{\mu_{\theta}}^{\overline{\mu_{n}}} \frac{1}{\nu(\mu)} d \mu=\int_{\sigma^{n}\left(\mu_{\theta}\right)}^{1} \frac{g^{\prime}(x)}{x} d x .
$$

In particular, $\sigma^{n}\left(\mu_{\theta}\right)>0$ for $\mu_{\theta}>\underline{\mu}^{19}$

Proof. Since $g^{\prime}(x) / x \geq 0$, there is a unique $\sigma^{n}\left(\mu_{\theta}\right)$ that solves (3) for each $\mu_{\theta}$, so integration establishes uniqueness and (3). See Appendix E.2 for details.

We now consider the sequence of economies $\mathcal{E}^{n}$ as $\overline{\mu_{n}} \rightarrow \infty$. Our goal is to apply Proposition 1 in this insurance setting. ${ }^{20}$ Corollary 2 suggests that a necessary condition for the equilibria $\left(p^{n}, \alpha^{n}\right)$ to converge is that the improper integral on the left-hand side of (3) converges. That is, for some $\mu \in[\underline{\mu}, \infty)$,

$$
\int_{\mu}^{\infty} \frac{1}{\nu\left(\mu^{\prime}\right)} d \mu^{\prime}<\infty
$$

Corollary (3) formalizes the result.

Corollary 3. Suppose (4) does not hold. Then, for each $x>0$, price diverges: $\lim _{n \rightarrow \infty} p^{n}(x)=$ $\infty$. For each $\mu_{\theta}$, coverage converges to zero: $\lim _{n \rightarrow \infty} \sigma^{n}\left(\mu_{\theta}\right)=0$. Moreover, $\mathcal{E}$ does not have an equilibrium.

\footnotetext{
${ }^{19}$ If $\nu(\underline{\mu})>0, \sigma^{n}(\underline{\mu})>0$.

${ }^{20}$ Lemma 1 (Appendix A.1) shows that the technical Condition (1) holds in insurance markets. While Proposition 1 requires knowledge of the limit equilibrium $(p, \alpha)$, this result is enough for the setting of simple insurance markets because the structure of equilibrium is known from Theorem 2.
} 
Proof. From Corollary 1, as $\overline{\mu_{n}} \rightarrow \infty$, we must have $\lim _{x \rightarrow 1} p^{n}(x)=\infty$. Each function $p^{n}(x)$ must also satisfy incentive compatibility. If (4) fails, $p^{n}(x)$ cannot be sufficiently convex to diverge at full insurance without also diverging at every other alternative. In that case, as $\overline{\mu_{n}} \rightarrow \infty$, all prices diverge and the allocation of every individual approaches $x=0$. In the limit, an AG equilibrium does not exist (because prices are not defined). See Appendix E.4 for details.

For "fragile" economies ((4) fails), relaxing the truncation of the cost distribution results in market unravelling. The price of each alternative $x>0$ increases without bound and the levels of coverage chosen by each type $\mu_{\theta}$ approaches zero. The limit economy $\mathcal{E}$ does not have an $\mathrm{AG}$ equilibrium.

For "fragile" unbounded economies, it is tempting to think that the equilibrium is "all types buy zero insurance." Indeed, the allocation $\sigma^{n}\left(\mu_{\theta}\right)$ converges to zero for each type. However, price $p^{n}(x)$ diverges for each contract. AG equilibrium requires that all prices be defined, so $\mathcal{E}$ has no equilibrium. Predicting market outcomes would require defining a new notion of equilibrium, which is outside the scope of this article.

When (4) fails, cost bounds have an unbounded effect on equilibrium prices. Notice that, if $\nu\left(\mu_{\theta}\right)=\nu_{0}$ is a constant (that is, individuals differ only in risk, as in RS), and cost is unbounded, then the economy is fragile. It is known that equilibria in RS (and other similar) settings can change discontinuously when the mass of a given type vanishes (Mailath et al. [1993]). Our contribution is to show formally that, in insurance markets, increasing the upper bound of cost results in full unravelling.

This result is illustrated in Figure 2. The left panel depicts a numerical simulation where $\nu$ is constant, so (4) fails. The figure shows the price functions $p^{n}(x)$ in the equilibrium of several truncated economies $\mathcal{E}^{n}$ with increasing values of the upper bound $\overline{\mu_{n}}$, showing that $p^{n}(x)$ diverges for each $x .^{21}$

Condition (4) is necessary for equilibrium existence, but also sufficient. When (4) holds, changes in the support of the type distribution have a bounded effect on equilibrium prices. Therefore, (4) creates a sharp distinction between economies that are robust and fragile w.r.t. cost bounds, as formalized by Corollary 4 .

Corollary 4. Suppose (4) holds. Then there exists a unique equilibrium $(p, \alpha)$ of $\mathcal{E}$, and the associated choice rule $\sigma:[\mu, \infty] \rightarrow[0,1)$ is defined by

$$
\int_{\mu_{0}}^{\infty} \frac{1}{\nu(\mu)} d \mu=\int_{\sigma\left(\mu_{0}\right)}^{1} \frac{g^{\prime}(x)}{x} d x .
$$

for any $\mu_{0}>\underline{\mu}$. In particular, $\sigma\left(\mu_{0}\right)>0$ for $\mu_{0}>\underline{\mu}_{.}^{22}$

\footnotetext{
${ }^{21} \mathrm{~A}$ graph of $\mu_{\theta}=\sigma^{-1}(x)$ would look similar (since each price $p$ is associated to a single cost $\mu_{\theta}$ ). This implies that, as $n \rightarrow \infty$, each type $\mu_{\theta}$ obtains progressively lower coverage in equilibrium.

${ }^{22}$ If $\nu(\underline{\mu})>0, \sigma(\underline{\mu})>0$.
} 

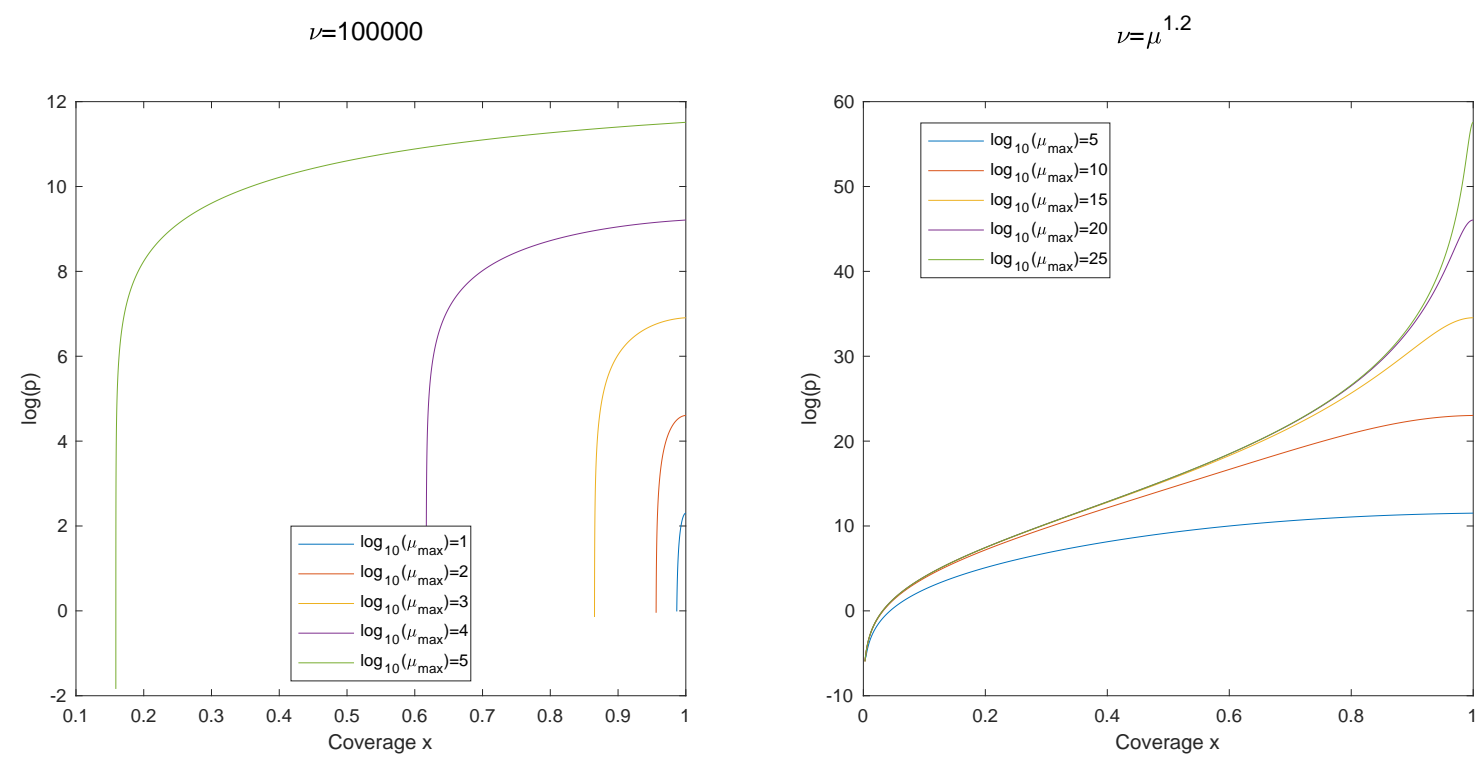

Figure 2: Left panel: equilibrium does not exist when $\nu$ constant. Right panel: equilibrium exists when $\nu_{\theta}=\left(\mu_{\theta}\right)^{1.2}$. The different curves correspond to the prices $p^{n}(x)$ of simulated economies where $\log _{10}\left(\overline{\mu_{n}}\right) \in\{1,2,3,4,5\}$ when $\nu_{\theta}$ constant and $\log _{10}\left(\overline{\mu_{n}}\right) \in$ $\{5,10,15,20,25\}$ when $\nu_{\theta}=\left(\mu_{\theta}\right)^{1.2}$.

Proof. By Condition (4), Proposition 1 holds, so equilibrium exists. Uniqueness and (5) follow since, for any $\bar{\mu}>\mu_{0}>\underline{\mu}$, we have $\int_{\mu_{0}}^{\bar{\mu}} \frac{1}{\nu(\mu)} d \mu=\int_{\sigma\left(\mu_{0}\right)}^{\sigma(\bar{g})} \frac{g^{\prime}(x)}{x} d x$. Taking $\bar{\mu} \rightarrow \infty$ gives the result, as $\lim _{\bar{\mu} \rightarrow \infty} \sigma(\bar{\mu})=1$ by Theorem 2. See Appendix E.3 for details.

The result is illustrated in Figure 2, where the right panel shows a setting where $\nu_{\theta}=$ $\left(\mu_{\theta}\right)^{1.2}$, so (4) holds. As the support of $\mu_{\theta}$ expands, the functions $p^{n}(x)$ converge to $p(x)$, and each type's choice $\sigma^{n}\left(\mu_{\theta}\right)$ converges to $\sigma\left(\mu_{\theta}\right)$.

How restrictive is (4)? It is satisfied, for instance, if $P$-a.s., $\nu_{\theta}$ grows asymptotically at least as fast as $C\left(\mu_{\theta}\right)^{\alpha}$ for some $C>0$ and $\alpha>1 .{ }^{23}$ In the CARA-Gaussian framework of Example 1, $\nu_{\theta}=a_{\theta} \sigma_{\theta}^{2}$ is the product of the CARA risk aversion coefficient $a_{\theta}$ and the variance of shocks $\sigma_{\theta}^{2}$. In this case, (4) holds if $a_{\theta}$ is constant and $\sigma_{\theta}^{2}$ increases more than linearly with the expected cost $\mu_{\theta}$. On the other hand, the result also shows that models where $\nu_{\theta}$ is constant necessarily describe "fragile" economies.

Condition (4) is empirically likely in markets like health and auto insurance where individuals with higher expected risk tend to have larger variance in outcomes, as described by Brown et al. [2014], Hendren [2013]. For instance, Handel et al. [2015] estimate the distribution of healthcare expenditures conditional on individual covariates and based on an empirical model with CARA utility. Those authors find (Table III of their article) that, as age increases, both the variance $\sigma_{\theta}^{2}$ and the mean $\mu_{\theta}$ increase, but the former increases faster than linearly w.r.t. the latter.

\footnotetext{
${ }^{23}$ Formally, $\lim \inf { }_{\mu \rightarrow \infty} \frac{\nu(\mu)}{\mu^{\alpha}}>0$, where the limit is taken along the support of $P_{\mu}$.
} 
Condition (4) fails, for instance, if for some $C, D \in \mathbb{R}, \nu_{\theta} \leq C \mu_{\theta}+D P$-a.s.. Section 6.5 provides a class of economies where this condition implies equilibrium non-existence, in a more general setting where $\theta=\left(\mu_{\theta}, \nu_{\theta}\right)$ may be truly two-dimensional.

\subsection{Discrete Types}

We now consider an insurance market with unbounded discrete types. Our goal is to illustrate features of the equilibrium familiar from RS and AG. For instance, $p(x)$ is the upper envelope of indifference curves and incentive compatibility constraints bind "downwards." We present heuristic arguments here, and proofs in Appendix F.

The unbounded economy $\mathcal{E}=[\Theta, X, P]$ has types $\Theta=\left\{\left(\mu_{k}, \nu_{k}\right)_{k=1}^{\infty}\right\}$ where $\mu_{k}, \nu_{k}$ are strictly increasing in $k$ and $\mu_{k} \rightarrow \infty, \nu_{k} \rightarrow \infty$. The alternative space is $X=[0,1)$. The truncated economy $\mathcal{E}^{n}=\left[\Theta^{n}, \bar{X}, P\left(\cdot \mid \Theta^{n}\right)\right]$ has types $\Theta^{n}=\left\{\left(\mu_{k}, \nu_{k}\right)_{k \leq n}\right\}$, alternative space $\bar{X}=[0,1]$ and distribution $P\left(\cdot \mid \Theta^{n}\right)=P\left(\cdot \cap \Theta^{n}\right) / P\left(\Theta^{n}\right)$.

First, we construct the unique equilibrium of $\mathcal{E}^{n}$. Type $\theta_{k}$ chooses $\left(x_{k}^{n}, p_{k}^{n}\right)$, where $x_{k}^{n}, p_{k}^{n}$ are strictly increasing in $k$. Each contract breaks even: $p_{k}^{n}=x_{k}^{n} \mu_{k}^{n}, \forall k$. Type $\mu_{k}^{n}$ is indifferent between $\left(x_{k}^{n}, p_{k}^{n}\right)$ and $\left(x_{k-1}^{n}, p_{k-1}^{n}\right)$. Together, these imply

$$
\frac{\mu_{k}^{n}-\mu_{k-1}^{n}}{\nu_{k}^{n}}=\frac{g\left(x_{k}^{n}\right)-g\left(x_{k-1}^{n}\right)}{x_{k-1}^{n}}
$$

which is the discrete analogue of (2). The highest-cost type $\mu_{n}^{n}$ obtains full insurance $(x=1)$ at a price $p_{n}^{n}=\mu_{n}^{n}$. Then, (6) pins down the value of $x_{n-1}^{n}$, then of $x_{n-2}^{n}$, and so forth. In fact, (6) implies that there exists a continuous function $\phi_{k}(\cdot)$ that determines any $x_{k}^{n}=\phi_{k}\left(x_{k+1}^{n}\right)$ based on knowledge of $x_{k+1}^{n}$, so equilibrium choices can be defined recursively. ${ }^{24}$ Let $\mathcal{I}_{k}^{n}:[0,1] \rightarrow \mathbb{R}$ be the indifference curve of type $\mu_{k}^{n}$ through her chosen contract, $\left(x_{k}^{n}, p_{k}^{n}\right) .{ }^{25}$ We define $p^{n}(x)=\mathcal{I}_{k}^{n}(x)$ if $x \in\left[x_{k-1}^{n}, x_{k}^{n}\right] .{ }^{26}$ Incentive compatibility requires $\mathcal{I}_{k}^{n}\left(x_{k}^{n}\right)=\mathcal{I}_{k+1}^{n}\left(x_{k}^{n}\right)$, so $p^{n}(\cdot)$ is continuous. ${ }^{27}$ Moreover, $\mathcal{I}_{k}^{n}\left(x_{k}^{n}\right)=\mu_{k} x_{k}^{n}$ so the break-even condition is satisfied. Notice that $p^{n}(x)$ is the upper envelope of indifference curves at each individual's chosen contract, as in AG. This construction is formalized in Proposition 2 which generalizes AG's Corollary 1 . Figure 3 provides a visual illustration of the equilibrium for a truncated economy with four cost types, $\mathcal{E}^{4}$.

Proposition 2. The truncated economy $\mathcal{E}^{n}$ has a unique equilibrium where price is $p^{n}(\cdot)$ and the allocation $\alpha^{n}$ is concentrated on $\left(\mu_{k}, \nu_{k}, x_{k}^{n}\right)_{k \leq n}$, with $\alpha^{n}\left(\left\{\left(\mu_{k}, \nu_{k}, x_{k}^{n}\right)\right\}\right)=\frac{P\left(\mu_{k}\right)}{P\left(\Theta^{n}\right)}$.

\footnotetext{
${ }^{24}$ We have $x_{k}^{n}:=\phi_{k}\left(\phi_{k+2}\left(\cdots\left(\phi_{n-1}(1)\right) \cdots\right)\right)$. For instance, in the CARA-Gaussian framework of Example $1, \phi_{k}(\cdot)$ is the positive solution of a second degree equation.

${ }^{25} \mathcal{I}_{k}^{n}$ is expressed algebraically by (17) in Appendix D.1.

${ }^{26}$ Let $x_{0}^{n}=\min \left[x \mid \mathcal{I}_{0}^{n}(x) \geq 0\right]$, and for convenience, set $x_{-1}^{n}=0$ and $\mathcal{I}_{0}^{n}=0$.

${ }^{27}$ Notice also that, for $x \leq x_{k}^{n}$, the indifference curve is below the break even line $\left(\mathcal{I}_{k}^{n}(x) \leq \mu_{n} x_{k}^{n}\right)$, as shown in Figures 1 and 3.
} 


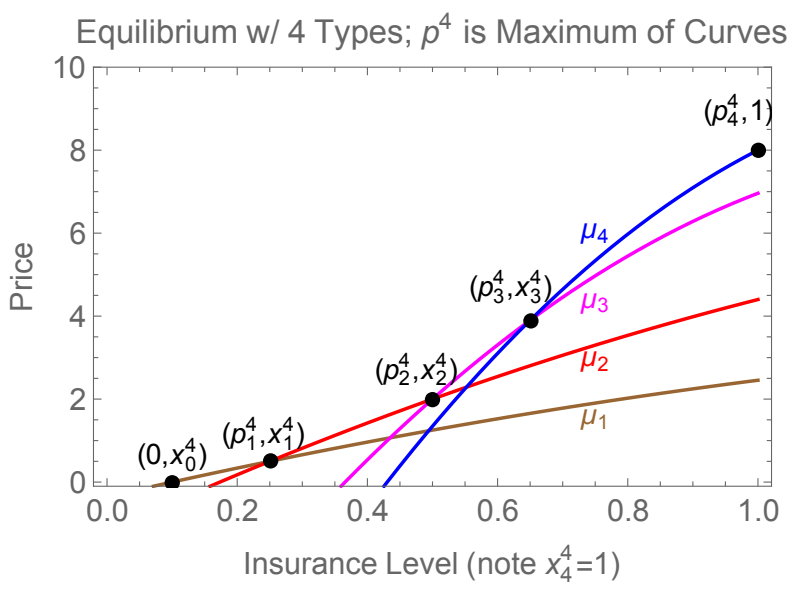

Figure 3: Construction of the Price Function $p^{4}(x)$ for $\mathcal{E}^{4}$. For each interval $x \in\left[x_{k-1}^{4}, x_{k}^{4}\right]$, prices are given by $p^{4}(x)=\mathcal{I}_{k}^{4}(x)$ where $\mathcal{I}_{k}^{4}(x)$ is the indifference curve of type $k$, who chooses $\left(x_{k}, p_{k}\right)$. The price $p^{4}(x)$ corresponds to the upper envelope of the indifference curves of buyers.

Proof. Follows from Theorem $2 .^{28}$

We now construct the equilibrium of the limit economy $\mathcal{E}$. Appendix F shows $\left(x_{k}^{n}\right)_{n \geq k}^{\infty}$ is monotonically decreasing for each $k$, so $\lim _{n \rightarrow \infty} x_{k}^{n}=x_{k} \in[0,1)$ exists, and corresponds to the choice of type $k$ in $\mathcal{E}$. Continuity of $\phi_{k}(\cdot)$ implies incentive compatibility in $\mathcal{E}$, i.e. $x_{k}=\phi_{k}\left(x_{k+1}\right)$, as desired. The equilibrium allocation is $\alpha\left(\left\{\left(\mu_{k}, x_{k}\right)\right\}\right)=P\left(\mu_{k}\right)$. Let $\mathcal{I}_{k}:[0,1] \rightarrow \mathbb{R}$ be the indifference curve of $\mu_{k}$ through $\left(p_{k}, x_{k}\right)$, and piece these together as above to form $p(x)=\mathcal{I}_{k}(x)$ if $x \in\left[x_{k-1}, x_{k}\right]^{29}$

Since (6) is the discrete analogue of (2), it seems likely that a necessary condition for existence will be a discrete analogue of (4), namely

$$
\sum_{k=1}^{\infty} \frac{\mu_{k+1}-\mu_{k}}{\nu_{k+1}}<\infty
$$

Proposition 3 (which mirrors Corollary 3) shows this condition is indeed necessary.

Proposition 3. Suppose (7) does not hold. Then, for each $x>0$, price diverges: $\lim _{n \rightarrow \infty} p^{n}(x)=$ $\infty$. For each $k \in \mathbb{N}$, coverage converges to zero: $\lim _{n \rightarrow \infty} x_{k}^{n}=0$. Moreover, $\mathcal{E}$ does not have an equilibrium.

Proof. See Appendix F.

\footnotetext{
${ }^{28}$ To avoid logical circularity, the uniqueness stated in Theorem 2 is not be relied on here, but the allocations follow from the other properties, as the highest type purchases full insurance and the other types allocations are determined inductively.

${ }^{29}$ Again, $x_{0}=\min \left[x \mid \mathcal{I}_{0}(x) \geq 0\right]$, and set $x_{-1}=0$ and $\mathcal{I}_{0}=0$. Notice that $p(\cdot)$ is well-defined and continuous, and $p(x)=\max _{k \in \mathbb{N}} g_{k}(x)$. Also, $p$ involves 'infinitely many pieces' and hence is not defined at $x=1$.
} 


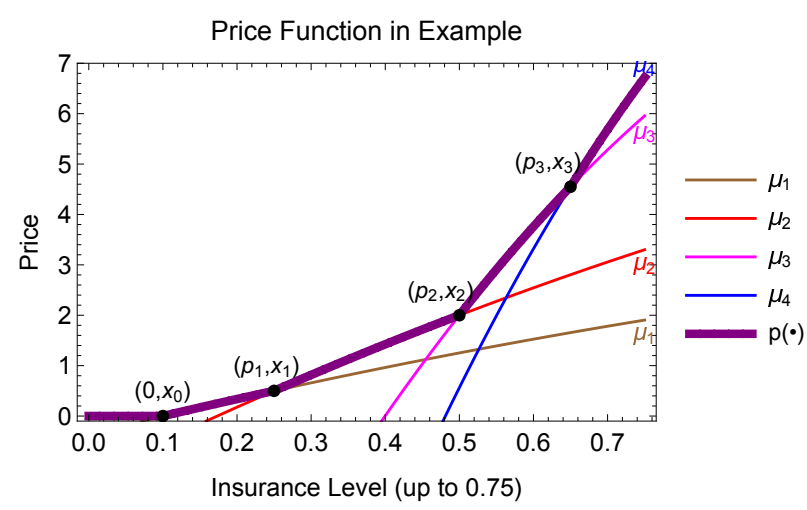

Figure 4: Construction of Price Function for the unbounded economy with discrete types. We show only the domain $x \in\left(0, \frac{3}{4}\right)$, since $\lim _{x \rightarrow 1} p(x)=\infty$.

If (7) fails, relaxing the truncation of $\mathcal{E}$ raises the price of each contract without bound and reduces each type's chosen coverage to zero. In the limit, equilibrium does not exist. In parallel to the analysis of economies with continuous types, (7) is also sufficient for existence.

Proposition 4. Suppose that (7) holds. Then $x_{k}>0$ for all $k \in \mathbb{N} ; p_{n} \rightarrow p$ uniformly on compact subsets of $[0,1)$, and $\alpha^{n} \rightarrow \alpha$ weakly. Therefore, by Proposition $1,(p, \alpha)$ is an equilibrium. Moreover, the equilibrium is unique.

Proof. We have $x_{k}>0$ for all $k \in \mathbb{N}$, which follows from (7) by calculation. Convergence follows from the definitions of $\alpha^{n}, \alpha, p^{n}, p$ in terms of the $x_{k}^{n}$, since $x_{k}^{n} \rightarrow x_{k}$ for each $k$, and since each compact subset of $[0,1)$ contains finitely many of the points $x_{n}$. For uniqueness, in any equilibrium, for each $k \in \mathbb{N}$, the contract $x_{k}$ purchased by type $\left(\mu_{k}, \nu_{k}\right)$ satisfies $x_{k}:=\lim _{n \rightarrow \infty} \phi_{k}\left(\phi_{k+2}\left(\cdots\left(\phi_{n}(1)\right) \cdots\right)\right)$ and that limit is well-defined. For details, see Appendix F.

Intuitively, the summability condition (7) is similar to the integrability condition (4). In the discrete case, $p(x)$ is the upper envelope of indifference curves, and the slope of these curves is $w_{\theta}(x)=\mu_{\theta}+(1-x) \nu_{\theta}$. For unbounded economies, $\lim _{x \rightarrow 1} p(x)=\infty$ by Proposition 10, so $w_{\theta}$ must increase sufficiently fast as $x \rightarrow 1$. Since the choice of $x$ increases with $\mu_{\theta}$, then $\nu_{\theta}$ must increase sufficiently fast to allow for $\lim _{x \rightarrow 1} p(x)=\infty$ without also arbitrarily raising prices for all $x<1$ (which would result in non-existence). The price function $p(\cdot)$ of the unbounded economy $\mathcal{E}$ is illustrated in Figure $4 .{ }^{30}$

\footnotetext{
${ }^{30}$ Appendix I also contains an alternative proof that $(p, \alpha)$ is an equilibrium of $\mathcal{E}$. This is a "direct" construction of the equilibrium which does not use Proposition 1.
} 


\section{Market for Lemons}

In this section we briefly consider markets for lemons, as in Akerlof [1970], where a single option is available. Several empirical studies assume a single or small number of options (e.g., Einav et al. [2010], Handel et al. [2015]), with estimation often assuming unbounded distributions for cost such as log-normal or truncated Gaussian. First, we give a condition on primitives such that equilibrium exists with unbounded cost. Then, we provide an example of non-existence when cost is unbounded. Therefore, non-existence is not an artifact resulting from a continuum of contracts.

We maintain the insurance framework of Section 4, including Assumption 1. There is a single non-zero product, so $X=\{0, \bar{x}\}$ for some $\bar{x}>0$. For simplicity, we assume that the insurance value $\nu=\nu(\cdot)$ is constant in this case, and assume $\mu_{\theta}$ distributes with positive PDF on some interval $(\underline{\mu}, \infty)$. The utility from purchasing non-zero (resp. zero) coverage at price $p$ for an agent of riskiness $\mu_{\theta}$ is $u_{\theta}=\bar{x} \mu_{\theta}+g(\bar{x}) \nu-p$ (resp. $u_{\theta}=-p$ ). ${ }^{31}$ The cost is $c(\theta, x)=x \mu_{\theta}$. When individuals do not buy $(x=0)$, their cost is zero: $c(\theta, 0)=$ $0, \forall \theta$.

By Theorem 1, an equilibrium exists if risk $\mu_{\theta}$ is bounded. The equilibrium (if it exists) must prescribe $p(0)=0$ and $p(\bar{x})$ equals the average cost of buyers. Let $p=p(\bar{x})$ denote the price of the non-zero alternative. If $\bar{x} \cdot \underline{\mu}+g(\bar{x}) \nu>\bar{x} \cdot \mathbb{E}[\mu]$, then charging a price $p=\bar{x} \cdot \mathbb{E}\left[\mu_{\theta}\right]$ and having all agents purchase is an AG equilibrium. Otherwise, let the marginal type (indifferent about purchasing) be $\mu^{\star}$ defined by $\bar{x} \mu^{\star}+g(\bar{x}) \nu=p$. The equilibrium price $p$ satisfies $p=\bar{x} \mathbb{E}\left[\mu_{\theta} \mid \mu_{\theta} \geq \mu^{\star}\right]$. If types are bounded, such a price $p$ always exists. Our existence result is as follows.

Proposition 5. If $\mu_{\theta}$ distributes with positive PDF on some interval $(\underline{\mu}, \infty)$ s.t.

$$
\lim _{M \rightarrow \infty} \mathbb{E}\left[\mu_{\theta} \mid \mu_{\theta} \geq M\right]-M=0
$$

then an equilibrium exists for any $\bar{x} \in(0,1]$ and any $\nu>0$. In particular, (8) holds if $\mu_{\theta}$ distributes with PDF $\phi$ and CDF $\Phi$ s.t. $\lim _{M \rightarrow \infty} \phi(M)=0$ and $\lim _{M \rightarrow \infty} \frac{1-\Phi(M)}{\phi(M)}=0$.

Proof. The condition $p=\mathbb{E}\left[\bar{x} \cdot \mu \mid \mu \geq \mu^{\star}\right]$ is equivalent to $\bar{x} \mu^{\star}+g(\bar{x}) \nu=\bar{x} \mathbb{E}[\mu \mid \mu \geq$ $\left.\mu^{*}\right]=\bar{x} \mu^{*}+\bar{x} \zeta\left(\mu^{*}\right)$, where $\zeta(z)=\mathbb{E}[\mu-z \mid \mu \geq z]$; hence, $\lim _{z \rightarrow \infty} \zeta(z)=0$ and $\zeta(\cdot)$ is a continuous function. The above equality is equivalent to $\frac{g(\bar{x})}{\bar{x}} \nu=\zeta\left(\mu^{*}\right)$; there exists such a point, or $\zeta(\underline{\mu})<\frac{g(\bar{x})}{\bar{x}} \nu$, in which case, as remarked above, $p=\bar{x} \cdot E[\mu]$ is an equilibrium in which all types purchase coverage. The second part follows by L'Hospital's rule. ${ }^{32}$

Example 2. Suppose risk $\mu_{\theta} \geq 0$ distributes with a half-Gaussian distribution. That is,

\footnotetext{
${ }^{31}$ In this article, we do not consider existence when the space of alternatives is a fixed finite subset of $[0,1]$. That analysis is outside the scope of this article and left for future research.

${ }^{32}$ The authors are grateful to Ilan Nehama for pointing out this argument. Let $P(\cdot)$ denote distribution
} 
$\mu \sim|\tilde{\mu}|$ for $\tilde{\mu} \sim \mathcal{N}(0,1)$. Let $\Phi$ denote the CDF of $\mathcal{N}(0,1)$ and $\phi(x)=\sqrt{\frac{2}{\pi}} \cdot e^{-\frac{1}{2} x^{2}}$ the PDF of $\mu$ in $\mathbb{R}_{+}$. Notice that $\phi^{\prime}(x)=(-x) \cdot \phi(x)$, so by L'Hopital's law, $\lim _{M \rightarrow \infty} \frac{1-\Phi(M)}{\phi(M)}=$ $\lim _{M \rightarrow \infty} \frac{-\phi(M)}{\phi^{\prime}(M)}=\lim _{M \rightarrow \infty} \frac{1}{M}=0$. Therefore, an equilibrium exists.

We now provide two examples of non-existence. Equilibrium does not exist if every price is lower than the average cost of those who would purchase at that price, so firms are unable to break even at any price. Formally, $p<\mathbb{E}\left[\bar{x} \mu_{\theta} \mid \mu_{\theta} \geq \mu^{\star}\right]$. In this case, as in Section 4 , if the support of cost is progressively expanded, the share of individual who buys the contract $x=\bar{x}$ converges to zero.

Here again, it is tempting to conclude that "nobody buying" is an equilibrium. It is not, because AG equilibrium requires defining the price of all contracts. Since $p(\bar{x})$ diverges, an AG equilibrium does not exist. An alternative definition of equilibrium, which might exist under these circumstances, is left for future research.

Example 3. Suppose that $\mu_{\theta}$ has an exponential distribution with parameter $\frac{1}{\lambda}$, so that $\mathbb{E}\left[\mu_{\theta}\right]=\lambda$ and $\mathbb{E}\left[\mu_{\theta} \mid \mu_{\theta} \geq \mu^{\star}\right]=\mu^{\star}+\lambda$. Equilibrium does not exist if $\bar{x} \mu^{\star}+g(\bar{x}) \nu<$ $\bar{x}\left(\mu^{\star}+\lambda\right) \Leftrightarrow \frac{g(\bar{x})}{\bar{x}} \nu<\lambda$ (that is, if average cost $\lambda$ is large and risk aversion $\nu$ is low).

Example 4. Suppose that $\mu_{\theta}$ has a Pareto distribution with parameters $\mu_{0}$ and $\lambda>1 .{ }^{33}$ The conditional mean is $\mathbb{E}\left[\mu_{\theta} \mid \mu_{\theta} \geq \mu^{\star}\right]=\frac{\lambda}{\lambda-1} \mu^{\star}$ for any $\mu^{*} \geq \mu_{0}$. Equilibrium does not exist if $\bar{x} \mu^{\star}+g(\bar{x}) \nu<\bar{x} \frac{\lambda}{\lambda-1} \mu^{\star} \Leftrightarrow \frac{g(\bar{x})}{\bar{x}} \nu<\frac{1}{\lambda-1}$ (that is, if risk aversion is sufficiently low).

\section{Generalization \& Variations}

In this section, we first present a variant of Proposition 1, which applies when the limit equilibrium is not known. Then, we extend several results from Section 4 to more general insurance markets. This section can be skipped without loss of comprehension.

\subsection{Existence When the Limit Equilibrium is Unknown}

Proposition 1 requires knowledge of the equilibrium $(p, \alpha)$ of the limit economy $\mathcal{E}$. Proposition 6 does not require such knowledge but requires stronger assumptions. Like in Proposition 1 consider a sequence of compact subsets $\Theta^{1} \subseteq \Theta^{2} \subseteq \cdots \subseteq \Theta$ with $\cup_{n} \Theta^{n}=$ $\Theta$, such that for each $n \in \mathbb{N}$, each $\theta \in \Theta^{n}$ and each $\theta^{\prime} \in \Theta \backslash \Theta_{n}$, we have $c(\theta, \cdot) \leq c\left(\theta^{\prime}, \cdot\right)$.

of types, and $Y^{+}=\max [Y, 0]$. Then,

$$
\begin{aligned}
& E[\mu \mid \mu \geq M]-M=E[\mu-M \mid \mu-M \geq 0]=\frac{E\left[(\mu-M)^{+}\right]}{P(\mu \geq M)}=\frac{\int_{0}^{\infty} P\left((\mu-M)^{+} \geq x\right) d x}{P(\mu \geq M)} \\
& \quad=\frac{\int_{0}^{\infty} P\left((\mu-M)^{+} \geq x\right) d x}{P(\mu \geq M)}=\frac{\int_{M}^{\infty} P(\mu \geq x) d x}{P(\mu \geq M)}=\frac{\int_{M}^{\infty}(1-\Phi(x)) d x}{1-\Phi(M)} \underset{M \rightarrow \infty}{\rightarrow} \lim _{M \rightarrow \infty} \frac{1-\Phi(M)}{-\phi(M)}=0
\end{aligned}
$$

${ }^{33}$ That is, $\mu_{\theta}$ is concentrated on $\left(\mu_{0}, \infty\right)$ with PDF $f\left(\mu_{\theta}\right)=\lambda \mu_{0}^{\alpha}\left(\mu_{\theta}\right)^{-\lambda-1}$ and $\operatorname{CDF} F\left(\mu_{\theta}\right)=1-\left(\mu_{0} / \mu_{\theta}\right)^{\lambda}$. 
Proposition 6. Assume that, for every two alternatives $x, y \in X$, price $p \geq 0$, and type $\theta \in$ $\Theta$, there is price $q$ high enough s.t. $u(\theta, x, p)>u(\theta, y, q)$. Moreover, for each $n$, let $\left(p^{n}, \alpha^{n}\right)$ be an equilibrium of $\mathcal{E}^{n}$ such that the following conditions hold. First, the collection $\left(p^{n}\right)_{n=1}^{\infty}$ is point-wise bounded and equicontinuous in $X .{ }^{34}$ Second, for every $M \in \mathbb{R}$, there is a compact subset $K$ of $X$, s.t. $\inf _{x \notin K} p^{n}(x) \geq M$ for all n large enough. Third, Condition (1) holds. Then, there exists an equilibrium $(p, \alpha)$ of the unbounded economy $\mathcal{E}$, which is the limit of a subsequence of the equilibria $\left(p^{n}, \alpha^{n}\right)_{n=1}^{\infty}$ in the sense of Proposition 1.

Proof. See Online Appendix K.

The first assumption of Proposition 6 holds if $\sup _{p \geq 0} \frac{\partial u}{\partial p}(\theta, x, p)<0$ for each $\theta \in \Theta, x \in$ $X$. We use Proposition 6 below to derive existence results in insurance markets more general than those of Section $4 .{ }^{35}$

\subsection{General Insurance Markets Setup}

We now describe a more general insurance setting, where Assumptions 1,2 and 3 need not hold. We maintain the notation $\mu_{\theta}=\mu(\theta)=\mathbb{E}\left[Z_{\theta}\right]$, and the parameterization that alternative $x$ covers a share $x \in[0,1]$ of cost, so $c(\theta, x)=x \mu_{\theta}$. We require that utility $u_{\theta}(x, p)$ is defined for all $x \in[0,1]$ and all $p \in \mathbb{R}_{+}$. Unless otherwise specified, we take $X=[0,1]$ or $X=[0,1)$.

We now list several assumptions which will be used in the remainder of this section. Notice that not all assumptions are needed for each result.

Assumption 4. Utility is quasilinear in price: $u_{\theta}(x, p)=x \mu_{\theta}+g_{\theta}(x)-p$, with $g_{\theta}:[0,1] \rightarrow \mathbb{R}$ smooth, strictly increasing and concave $\left(g_{\theta}^{\prime}(x)>0, g_{\theta}^{\prime \prime}(x)<0\right.$ for $\left.x \in(0,1)\right)$, with $g_{\theta}^{\prime}(1)=0$.

Assumption 4 generalizes Assumption 1, where it was required that $g_{\theta}(x)=g(x) \nu_{\theta}$, but still maintains quasi-linearity in price. Under Assumption 4, the marginal willingness to pay for additional insurance is $w_{\theta}(x)=\frac{\partial u_{\theta}}{\partial x}(x, p)=\mu_{\theta}+g_{\theta}^{\prime}(x) \geq \mu_{\theta}$ with equality if and only if $x=1$. By quasi-linearity, $w_{\theta}$ is independent of price. ${ }^{36}$

Assumption 5. Utility is $u: \Theta \times[0,1] \times \mathbb{R}_{+}$is continuous, $u_{\theta}(\cdot, \cdot)$ is twice differentiable for all $\theta \in \Theta$ and the second derivatives are continuous in $(\theta, x, p)$, with $\frac{\partial u_{\theta}}{\partial x}>0, \frac{\partial u_{\theta}}{\partial p}<0$, and $w_{\theta}(x, p)=-\frac{\partial u_{\theta}}{\partial x}(x, p) / \frac{\partial u_{\theta}}{\partial p}(x, p)$ satisfies $w_{\theta} \geq \mu_{\theta}$ with equality iff $x=1$, and $\frac{\partial w_{\theta}}{\partial x} \leq 0$ in $(0,1)$.

\footnotetext{
${ }^{34} \mathrm{~A}$ collection of real-valued functions $\mathcal{F}$ on a metric space $(X, d)$ is point-wise bounded if $\forall x \in X$, $\sup _{f \in \mathcal{F}}|f(x)|<\infty$. Moreover, $\mathcal{F}$ is equicontinuous if, for each $\varepsilon>0$ and each $x \in X$, there is $\delta>0$ such that if $y \in X$ with $d(x, y)<\delta$, then $|f(y)-f(x)|<\varepsilon$ for all $f \in \mathcal{F}$.

${ }^{35}$ Note that the first condition required in Proposition 6 (one option is preferred over another if the latter's price is high enough) holds when utility is quasi-linear in price, as in Section 4.

${ }^{36}$ Assumption 4 also guarantees the condition on utilities required by Proposition 6 .
} 
Assumption 5 is an alternative assumption on utility functions. Note that, under Assumption 5, $w_{\theta}$ may depend on both coverage and price.

Assumption 6. For each $(x, p) \in[0,1] \times \mathbb{R}_{+}, w_{\theta_{2}}(x, p) \geq w_{\theta_{1}}(x, p)$ iff $\mu\left(\theta_{2}\right) \geq \mu\left(\theta_{1}\right)$.

Assumption 6 generalizes Assumption 2. If Assumption 6 holds, all types with the same riskiness have the same utility function, so we may view $\underline{\nu}_{\delta}$ as a function of $\mu_{\theta}$, defined on the support of $P_{\mu}$. That is, types are effectively one-dimensional. ${ }^{37}$ We remark:

Proposition 7. Assume Assumption 5; further assume $\Theta$ is an open subset of a Euclidean space s.t. the maps $\theta \rightarrow \mu(\theta)$ and $\theta \rightarrow w_{\theta}(x, p)$ are everywhere non-singular (i.e., non-zero gradient). Then Assumption 6 holds iff single crossing holds, i.e., iff indifference curves of types (whose preferences are not identical) intersect at most once.

We remark that the topological and regularity assumptions in the proposition can be relaxed, and we have merely assumed 'comfortable' assumptions for illustrative purposes.

Proof. The assumptions on $\Theta, \mu, w$ imply a continuous type space and that the costs and marginal willingness to pay possess some regularity. It is immediate that Assumption 6 implies single crossing. To show the converse, suppose $\mu\left(\theta_{2}\right)>\mu\left(\theta_{1}\right)$ but, by way of contradiction, there were some $x_{0}, p_{0} \in[0,1] \times \mathbb{R}_{+}$at which $w_{\theta_{2}}\left(x_{0}, p_{0}\right)<w_{\theta_{1}}\left(x_{0}, p_{0}\right)$. Then, the indifference curve of $\theta_{1}$ through $\left(x_{0}, p_{0}\right)$ at full coverage is, by single crossing, higher than the indifference curve of $\theta_{2}$ through $\left(x_{0}, p_{0}\right)$ at full coverage. As we move the 'pivot point' $\left(x_{0}, p_{0}\right)$ along the latter curve towards higher coverage, this reality must flip at some point because, near full coverage, $w_{\theta_{2}} \approx \mu\left(\theta_{2}\right)>w_{\theta_{1}}\left(x_{0}, p_{0}\right) \approx \mu\left(\theta_{1}\right)$. However, by single-crossing, if indifference curves intersect at $\left(x_{0}, p_{0}\right)$ they do not intersect elsewhere, which yields a contradiction. Formal details are in Online Appendix K.

Assumption 7. For every $\mu^{*}>\mu$, the $\operatorname{set}\left\{\theta \mid \mu(\theta) \leq \mu^{*}\right\}$ is compact in $\Theta$.

Assumption 7 implies that, for each upper bound on cost, only a compact set of types have cost below this bound. Like Assumption 6, Assumption 7 rules out unbounded distributions like (for instance, Gaussian and log-normal). We know of no case in which Assumption 6 holds and Assumption 7 does not, so Assumption 7 is with a small additional loss of generality. ${ }^{38}$

\footnotetext{
${ }^{37}$ Recall $\mu=\min \left(\operatorname{supp}\left(P_{\mu}\right)\right)$. If $(a, b)$ is a maximal open interval not intersecting the support of $P_{\mu}$, (that is, there are no types with riskiness $\mu$ in $(a, b)$, but $\left.a, b \in \operatorname{supp}\left(P_{\mu}\right)\right)$, we interpret $\underline{\nu}_{\delta}(\mu)=\underline{\nu}_{\delta}(a)$ in $(a, b)$. That is, we complete the function $\underline{\nu}_{\delta}$ from the domain $\operatorname{supp}\left(P_{\mu}\right)$ to $[\mu, \infty)$ such that $\underline{\nu}_{\delta}(\mu)=\nu_{\delta}(\max \{\zeta \in$ $\left.\left.\operatorname{supp}\left(P_{\mu}\right) \mid \zeta \leq \mu\right\}\right)$.

${ }^{38} \mathrm{~A}$ weakening of Assumption 7 which is sufficient for all our purposes, including Proposition 8 , is given in Online Appendix J; this weakening would make the analysis at some points significantly lengthier.
} 


\subsection{Equilibrium Characterization}

Proposition 8 generalizes Theorem 2.

Proposition 8. Suppose that Assumptions 5, 6, and 7 hold. Any equilibrium $(p, \alpha)$ of the bounded $(X=[0,1])$ or unbounded $(X=[0,1)$ ) insurance economy $\mathcal{E}=[\Theta, X, P]$, satisfies:

1. Price $p(x)$ is continuous and strictly increasing on the domain on which it is nonzero (Lemma 11).

2. There is a continuous mapping $\sigma: \operatorname{supp}\left(P_{\mu}\right) \rightarrow[0,1]$, strictly increasing on the domain in which it is non-zero, that assigns to each type $\mu_{\theta}$ her chosen contract. Formally, $\alpha\left\{\left(\mu_{\theta}, x\right) \mid x=\sigma\left(\mu_{\theta}\right)\right\}=1 .^{39}$ (Lemma 6)

3. Contracts are actuarily fair. Formally, $P-$ a.s., $p\left(\sigma\left(\mu_{\theta}\right)\right)=\mu_{\theta} \sigma\left(\mu_{\theta}\right)$. (Lemma 4)

4. Full insurance is in the support of the equilibrium..$^{40}$ (Corollary 7 )

5. Let $x_{0}<1$. If $L \geq w_{\theta}$ in $\left\{x \leq x_{0}, p \leq p\left(x_{0}\right)\right\}$ for a.e. $\theta$ which chooses coverage up to $x_{0}$ (i.e., $\alpha\left(\cdot \mid x \leq x_{0}\right)-a . s$.) then $p(\cdot)$ is $L$-Lipshitz in $\left[0, x_{0}\right] .{ }^{41}$ Formally, we may take $L=e s s-\sup \left\{\max _{\left\{x \leq x_{0}, p \leq p\left(x_{0}\right)\right\}}\left(w_{\theta} \mid \sigma\left(\mu_{\theta}\right) \leq x_{0}\right)\right\} .{ }^{42}$

6. Let $x \in(0,1]$ and $p(x)>0$. Let $\eta\left(\mu_{\theta}, x\right)$ be the price at which type $\mu_{\theta}$ is indifferent between his purchased contract $\left(\sigma\left(\mu_{\theta}\right), p\left(\sigma\left(\mu_{\theta}\right)\right)\right.$ and $\left(x, \eta\left(\mu_{\theta}, x\right)\right)$. Then, for every $y>x$ for which $\alpha_{X}((x, y])>0$,

$$
p(x)=\operatorname{ess}-\sup \left\{\eta\left(\mu_{\theta}, x\right) \mid x<\sigma\left(\mu_{\theta}\right) \leq y\right\}
$$

That is, price is an envelope of indifference curves, and in fact one can take just those indifference curves of types who buy the 'next alternatives above it'. ${ }^{43}$

7. If in addition Assumption 3 holds, then 0 is $\alpha$-a.s. never purchased, and in particular $\sigma: \operatorname{supp}\left(P_{\mu}\right) \rightarrow[0,1]$ is strictly increasing.

Proof. See Appendix C.

\footnotetext{
${ }^{39}$ Under Assumptions 1, 2, and 3, we show that $x=0$ is never chosen in equilibrium (see Sections 4.3 and 4.4). However, we have not been able to show this in more general settings. Therefore, we only assert that $\sigma$ is strictly increasing on $\operatorname{supp}\left(P_{\mu}\right) \backslash \sigma^{-1}(\{0\})$.

${ }^{40}$ The support of a positive measure is the smallest closed non-null set. Therefore, we mean that every neighborhood of $x=1$ has strictly positive measure under $\alpha$.

${ }^{41}$ If $\Theta$ is compact, this implies that the price function is Lipshitz.

${ }^{42}$ Recall that the essential supremum ("ess - sup") of a random variable $X$ with distribution $P$ is the supremum over all $x \in \mathbb{R}$ s.t. $P(X>x)>0$; intuitively, this is the 'supremum up to measure zero'.

${ }^{43}$ In particular, if $\mu_{1}<\mu_{2}$ are atoms of $P_{\mu}$ but there are $\alpha$-a.s. no types with riskiness between $\mu_{1}, \mu_{2}$, then type $\mu_{2}$ is indifferent between $\left(\sigma\left(\mu_{2}\right), p\left(\sigma\left(\mu_{2}\right)\right)\right.$ and the alternative "just below", $\left(\sigma\left(\mu_{1}\right), p\left(\sigma\left(\mu_{1}\right)\right)\right.$. This follows by taking $x=\sigma\left(\mu_{1}\right), y=\sigma\left(\mu_{2}\right)$ in the first part.
} 


\subsection{Existence in Insurance Markets}

We now generalize the existence results of Sections 4.3 and 4.4. The result uses Assumptions 4 and 6 and therefore requires that types be effectively one-dimensional.

First, we introduce some notation. For a given $\delta>0$,

$$
\bar{\nu}_{\delta}(\theta):=\max \left\{-\frac{\partial w_{\theta}}{\partial x}(x) \mid x \in[1-\delta, 1]\right\}, \underline{\nu}_{\delta}(\theta):=\min \left\{-\frac{\partial w_{\theta}}{\partial x}(x) \mid x \in[1-\delta, 1]\right\}
$$

Intuitively, $\bar{\nu}_{\delta}(\theta)$ (resp. $\underline{\nu}_{\delta}(\theta)$ ) is the highest (resp. lowest) level of curvature, in $(x, p)$ space, of type $\theta$ among contracts with generosity $x \in x \in[1-\delta, 1]{ }^{44}$ Notice that $-\frac{\partial w_{\theta}}{\partial x}(x)=-\frac{\partial^{2} u_{\theta}}{\partial x^{2}}(x)>0$ is the curvature of indifference curves in $(x, p)$-space for type $\theta$. If Assumption 6 holds, riskiness determines type, and we may write $\bar{\nu}_{\delta}(\mu), \underline{\nu}_{\delta}(\mu)$ instead; these are defined $P_{\mu}=P \circ \mu^{-1}$-a.s.

Proposition 9. Suppose that Assumptions 4, 6, and 7 hold. Suppose that for some $\delta>0$,

$$
\int_{\underline{\mu}}^{\infty} \frac{1}{\underline{\nu}_{\delta}(\mu)} d \mu<\infty .
$$

(the integrand assumes the maximal value of the two endpoints on any interval which has measure zero under $P_{\mu}$ ). Then, $\mathcal{E}$ possesses an equilibrium. When $P_{\mu}$ is purely atomic and supported on $\mu_{1}<\mu_{2}<\mu_{3}<\cdots$, the required condition is $\sum_{n=1}^{\infty} \frac{\mu_{n+1}-\mu_{n}}{\underline{\underline{\nu}}_{\delta}\left(\mu_{n}\right)}<\infty$.

Proof. See Online Appendix G.

\subsection{Unbounded Price and Existence}

We now generalize Corollary 1 . We also use it to describe a more general class of unbounded economies where an equilibrium does not exist. The results in this subsection do not require $X=[0,1)$, instead requiring only that $X \subseteq[0,1)$ be Borel ${ }^{45}$ such that full insurance is a limit point of $X{ }^{46}$ Importantly, the results also do not require that $\nu_{\theta}$ is a function of $\mu_{\theta}$ (Assumption 6), so types can be truly multi-dimensional and different types may pool within each contract. First, we elaborate an assumption needed for our result. ${ }^{47}$

Assumption 8. For each $\mu$, there is a $\bar{\delta}>0$ such that $\bar{\nu}_{\bar{\delta}}$ can be bounded as a function of $\mu$. Formally, for each $\bar{\mu}$ s.t. $P\left(\mu_{\theta} \leq \bar{\mu}\right)>0$, there is $\bar{\rho}=\bar{\rho}_{\bar{\delta}}(\bar{\mu})$ s.t. $P\left(\bar{\nu}_{\bar{\delta}}(\theta) \leq \bar{\rho} \mid \mu \leq \bar{\mu}\right)=1$.

\footnotetext{
${ }^{44}$ In the CARA-Gaussian framework of Example $1, \bar{\nu}_{\delta}(\theta)=\underline{\nu}_{\delta}(\theta)=\nu_{\theta}$ is independent of $\delta$.

${ }^{45}$ We require $X$ be Borel to be able to define a Borel measure on $X$. As Section 4.4 hints, a countable discrete set is often appropriate.

${ }^{46}$ I.e., there is a sequence $\left(x_{n}\right)$ in $X$ with $x_{n} \rightarrow 1$ but $\forall n, x_{n}<1$. We require that $x=1$ is a limit point of $X$ in order to prove that full insurance is not an atom of the equilibrium, in Proposition 10.

${ }^{47}$ Recall the notation $\bar{\nu}_{\delta}$ defined in (10).
} 
Assumption 8 constrains the joint distribution of $\mu_{\theta}$ and $\nu_{\theta}$. WLOG, we can take $\bar{\rho}_{\bar{\delta}}(\cdot)$ increasing and right-continuous. ${ }^{48}$ This assumption, while allowing for unbounded $\mu$, does not allow the distribution of $(\mu, v)$ to be arbitrary (for instance, bivariate Gaussian or lognormal), although such distributions can be approximated arbitrarily closely.

Proposition 10. Suppose Assumptions 4 and 8 hold, and that $P_{\mu}$ is not compactly supported. Then, in any equilibrium, $\lim _{x \rightarrow 1} p(x)=\infty$. In particular, $1 \notin X$, i.e., full insurance is not a possible contract.

Proof. First, we use Assumption 8 to establish bounds on the slope of $p(x)$ in equilibrium. Second, we show that full insurance cannot be an atom of an equilibrium (unless $x=1$ is bought by a single atomic type). Third, we show that smaller and smaller neighborhoods of full insurance attract arbitrarily large risks. Fourth, we use this to show that the price cannot be bounded in equilibrium. See Appendix D for details.

Assumption 8 is not a significant restriction on the result's implications. Any distribution is arbitrarily well approximated by a distribution satisfying Assumption $8 .^{49}$ Therefore, the result should be understood as saying that no economy satisfying Assumption 4 on utility and $P_{\mu}$ not compactly supported, can possess an equilibrium with bounded prices which is robust in any reasonable sense.

In Section 4, we mentioned that Condition (4) would not hold if $\nu \leq C \mu^{\alpha}+D$ with $\alpha \leq 1$. Proposition 10 describes a more general class of economies in which equilibrium prices must be unbounded. We now show that, under these more general conditions, such a bound will imply non-existence of equilibrium. The result requires only Assumption 4, so types are allowed to be "truly" multidimensional.

Proposition 11. Under Assumption 4, if $P_{\mu}$ is not compactly supported, and for some $\delta, C, D>0 P$-a.s., $\bar{\nu}_{\delta}(\theta) \leq C \cdot \mu(\theta)+D$, there exists no AG equilibrium.

Proof. Note that the assumption of the proposition implies Assumption 8. Intuitively, the bound on slopes implies that price cannot increase quickly enough to be unbounded, contradicting Proposition 10. See Appendix D.5.

In the framework of Section 4.1, Proposition 11 applies if $\nu_{\theta}$ is bounded by a linear function of $\mu_{\theta}$. This includes the case of consumers homogeneous in risk aversion (as in RS) but also applies in settings where types are truly multidimensional.

\footnotetext{
${ }^{48}$ If $\bar{\rho}_{\bar{\delta}}(\cdot)$ is not monotonic, it can just be replaced with its 'monotonic closure', $x \rightarrow \sup _{0 \leq y \leq x} \bar{\rho}_{\bar{\delta}}(y)$. If it is not right-continuous, it can be replaced with $x \rightarrow \lim _{y \rightarrow x^{+}} \bar{\rho}_{\bar{\delta}}(y)$. Both of these operations preserve linear growth, which is what we require in Proposition 11.

${ }^{49}$ Suppose, for instance, that the distribution of types $f^{\star}(\theta)$, for which 8 did not hold, resulted in a bounded equilibrium price $p^{\star}(\cdot)$. Approximating $f^{\star}(\theta)$ arbitrarily closely with a distribution $f(\theta)$ for which 8 holds, would result in some unbounded equilibrium prices $p($.$) which would be arbitrarily different from$ $p^{\star}(\cdot)$.
} 


\section{Conclusion}

The analysis of insurance markets in RS and AG is sensitive to assumptions on the bounds of the cost distribution. We show that, when assumptions on these bounds are fully relaxed, AG equilibria need not exist. We also provide sufficient conditions for equilibrium to exists when costs are unbounded.

In the context of insurance markets, we derive new properties of equilibrium that generalize AG and RS. We also present a necessary and sufficient condition for the existence of a unique equilibrium. We are able to derive a characterization of equilibrium for insurance markets with an unbounded continuum of types which is particularly tractable and based on a simple differential equation. We also show that, when it exists, the equilibrium features unbounded price at full insurance.

There are a number of interesting avenues for future research. First, our characterizations of equilibrium for simple insurance markets required a number of assumptions on utilities, contracts, and the distribution of types. In particular, several of our results require that types effectively be one-dimensional. Relaxing these assumptions would be an interesting avenue for future research. Second, in this article, we have focused on the AG equilibrium concept, which exists in a wide range of screening markets. It would be interesting to replicate the exercise in this paper to other equilibrium concepts, such as those of Miyazaki [1977], Wilson [1977], Spence [1978], Riley [1979]. ${ }^{50}$ Third, we also do not consider existence when the space of alternatives is a fixed finite subset of $[0,1]$ (for instance, as in Handel et al. [2015] who consider two contracts). This analysis is outside the scope of this article and also left for future research.

\section{Appendix}

\section{A Existence in Unbounded Economies}

\section{A.1 Proposition 1}

Given the definition of AG equilibrium in Section 2, we say that $\left(\left(\bar{X}_{j}\right),\left(p_{j}, \alpha_{j}\right),\left(\eta_{j}\right)\right)$ witnesses that $(p, \alpha)$ is an equilibrium.

For each $n$, let $\bar{X}_{n}$ be a Polish space in which $X$ is dense and let $\left(Y_{n}^{k}\right)_{k \in \mathbb{N}},\left(\zeta_{n}^{k}\right)_{k \in \mathbb{N}}$, $\left(q_{n}^{k}, \beta_{n}^{k}\right)_{k \in \mathbb{N}}$ be sequences finite sets of alternatives in $\bar{X}_{n}$, of distributions of behavioral

\footnotetext{
${ }^{50}$ These equilibrium concepts are not guaranteed to exist in general. For instance, Azevedo and Gottlieb [2016] provide an example of non-existence of Riley [1979] equilibrium. Gemmo et al. [2018] shows existence of the Miyazaki [1977], Wilson [1977], Spence [1978], Riley [1979] equilibrium for continuous type distributions.
} 
types, and of weak equilibria which witness that $\left(p_{n}, \alpha_{n}\right)$ is an equilibrium of the restricted economy $n$. Note that $q_{n}^{k}$ refers to the price function while $\beta_{n}^{k}$ refers to the distribution over types and contracts. For each $n \in \mathbb{N}, \bar{X}_{n} \subseteq \bar{X}$, where $\bar{X}$ is a fixed compactification of $X .{ }^{51}$. Note that $Y_{n}^{k}$ generally includes points of $\bar{X}$ which are not in $X$.

Let $\left(Z_{j}\right)_{j \in \mathbb{N}}$ be a sequence of compact subsets of $X$ with $X=\cup_{j} Z_{j}$, and for each $j \in \mathbb{N}$, $Z_{j} \subseteq \operatorname{int}\left(Z_{j+1}\right)$. Such exists as $X$ is locally compact and separable. Note that since each $p_{n}(\cdot)$ is continuous (Lemma 16 , Online Appendix $\mathrm{H}$ ), $X$ is locally compact, and $p_{n} \rightarrow p$ uniformly on compact sets, then $p$ is continuous. WLOG, since each $Y_{n}^{n}$ is finite and $p_{n} \rightarrow p$ on uniformly on compact sets in $X$, we may assume ${ }^{52}$ there there are indices $\left(k_{n}\right)_{n}$ such that (after passing possibly to a sub-sequence of $\left(p_{n}, \alpha_{n}\right)$ ):

- For all $n \in \mathbb{N}$ and all $x \in Z_{n},\left|p_{n}(x)-p(x)\right|<\frac{1}{n}$.

- For all $n \in \mathbb{N}$ and all $x \in Y_{n}^{k_{n}},\left|q_{n}^{k_{n}}(x)-p_{n}(x)\right|<\frac{1}{n}$.

- For all $n \in \mathbb{N}, d\left(\beta_{n}^{k_{n}}, \alpha^{n}\right)<\frac{1}{n}$, where $d(\cdot, \cdot)$ is a metric for the weak topology.

Now, denote $\gamma_{n}=\beta_{k_{n}}^{n}, r_{n}=q_{n}^{k_{n}}, \bar{W}_{n}=Y_{n}^{k_{n}}$. Then $\gamma_{n}$ is concentrated on $\left(\Theta \cup \bar{W}_{n}\right) \times \bar{W}_{n}$, $\gamma_{n} \rightarrow \alpha$, and for all $n \in \mathbb{N},\left|r_{n}(x)-p(x)\right|<\frac{2}{n}$ for all $x \in \bar{W}_{n} \cap Z_{n}$. We contend that for each $x \in X$, and each sequence $\left(x_{n}\right)$ in $X$ with $x_{n} \rightarrow x$ and $x_{n} \in \bar{W}_{n}$ for each $n \in \mathbb{N}$, $r_{n}\left(x_{n}\right) \rightarrow p(x)$. Indeed, since $x \in X$ there is $N$ s.t. for all $n \geq N, x_{n} \in \operatorname{int}\left(Z_{n}\right)$; hence $x_{n} \in \operatorname{int}\left(Z_{n}\right) \cap \bar{W}_{n}$, so $\left|r_{n}\left(x_{n}\right)-p\left(x_{n}\right)\right|<\frac{2}{n}$, and since $p$ is continuous at $x, p\left(x_{n}\right) \rightarrow p(x)$; hence, $r_{n}\left(x_{n}\right) \rightarrow p(x)$.

We should like to say that the weak equilibria $\left(\gamma_{n}, r_{n}\right)$ witness that $(p, \alpha)$ is an equilibrium. The problem is that the marginal of $\gamma_{n}$ on $\Theta$ is $P\left(\cdot \mid \Theta_{n}\right)$, not $P$, so we must modify it. After adding the types in $\Theta \backslash \Theta_{n}$ to the distribution $\gamma_{n}$ (where each type is choosing a utility-maximizing option in $\bar{W}_{n}$ ), price can only go up, as elements outside $\Theta_{n}$ are more costly than those in it. Hence, by adding some behavioral types (who have cost 0 ) the added cost cancels out. ${ }^{53}$ For formal details, see Online Appendix K.

\section{A.2 Condition (1) holds in simple insurance}

Lemma 1. Under Assumption 1, Assumption 4, or Assumption 5, and where cost has the form $c(\theta, x)=\mu_{\theta} x$ and $\left.w_{\theta} \geq \mu_{\theta}\right)$, the condition (1) is satisfied when the other conditions of Proposition 1 or Proposition 6 hold.

Proof. First, we show there is $c_{0}>0$ and $x_{1} \in(0,1)$ s.t. $p_{n} \geq c_{0}$ in $\left\{x>x_{1}\right\}$ for $n$ large enough. In the case of Proposition 6, this is assumed. In the case of Proposition 1: Fix

\footnotetext{
${ }^{51}$ Formally, for each $n \in \mathbb{N}$ there is an embedding $\phi_{n}: \bar{X}_{n} \rightarrow \bar{X}$, which is identity on $X$.

${ }^{52}$ To show that the second bullet point may be assumed, see Lemma 17 of Online Appendix $H$.

${ }^{53}$ It is for these fine details that the assumption that types outside $\Theta_{n}$ have higher costs than those in $\Theta_{n}$, as well as Condition 1, is used.
} 
some $0<x_{0}<1$ for which $p\left(x_{0}\right)>0$; by the break-even condition and the continuity of $p$, such $x_{0}$ exists. Hence there is $c_{0}>0$, a neighborhood $U$ of $p\left(x_{0}\right)$, and $N$ s.t. if $n>N$, $p_{n} \geq c_{0}$ in $U$; since each $p_{n}$ is monotonic, this means that there is $x_{1}<x_{0}$ such that $p_{n} \geq c_{0}$ in $\left\{x>x_{1}\right\}$. Now, letting $\mu_{n}=\inf _{\Theta^{n} \backslash \Theta^{n-1}} \mu$, we have by assumption $\mu_{n} \rightarrow \infty$ monotonically; fix $N$ s.t. $\mu_{N} \geq \frac{p\left(x_{0}\right)}{x_{0}-x_{1}}$. Suppose by way of contradiction type $\theta \in \Theta \backslash \Theta^{N}$ purchases under $p^{n}$ coverage $x<x_{1}$. Since

$$
w_{\theta} \geq \mu_{N} \geq \frac{p\left(x_{0}\right)}{x_{0}-x_{1}}>\frac{p\left(x_{0}\right)-p(x)}{x_{0}-x},
$$

he must strictly prefer $\left(x_{0}, p\left(x_{0}\right)\right)$ to $(x, p(x))$, a contradiction.

\section{B Auxiliary Tool for Analyzing Allocations}

Fix a distribution $\alpha$ on $\Theta \times X$, with $X=[0,1]$ (e.g., $(p, \alpha)$ may be an equilibrium). Let marginal distribution of $\alpha$ on $\Theta$ be $P$. Let the marginal of $\alpha$ on $X$ be $\alpha_{X}$. We define analogues of the maximum and minimum risk $\mu$ purchasing each alternative $x$. To avoid the influence of zero-measure sets of types, we use a variation of the essential supremum and infimum, defined for $x \in \operatorname{supp}\left(\alpha_{X}\right)$ as ${ }^{54}$

$$
\begin{aligned}
& \psi^{+}(x)=\lim _{\delta \rightarrow 0^{+}}\left[\sup \left\{\mu \mid \alpha\left(\left\{\theta \mid \mu_{\theta} \geq \mu\right\} \times(x-\delta, x+\delta)\right)>0\right\}\right] \\
& \psi^{-}(x)=\lim _{\delta \rightarrow 0^{+}}\left[\inf \left\{\mu \mid \alpha\left(\left\{\theta \mid \mu_{\theta} \leq \mu\right\} \times(x-\delta, x+\delta)\right)>0\right\}\right] .
\end{aligned}
$$

Intuitively, $\psi^{+}(x)$ captures the largest value of $\mu$ which purchases $x$ under $\alpha$, and $\psi^{-}(x)$ as the lowest such value of $\mu$.

Note that either of these quantities can, a priori, be infinite. Moreover, note that for each $A \subseteq \operatorname{supp}\left(\alpha_{X}\right)$,

$$
\begin{aligned}
& \sup \left\{\mu \mid \alpha\left(\left\{\theta \mid \mu_{\theta} \geq \mu\right\} \times A\right)>0\right\} \leq \sup _{x \in A} \psi^{+}(A) \\
& \inf \left\{\mu \mid \alpha\left(\left\{\theta \mid \mu_{\theta} \leq \mu\right\} \times A\right)>0\right\} \geq \inf _{x \in A} \psi^{-}(A)
\end{aligned}
$$

Therefore, by comparing the left-hand terms of the following expressions to the lefthand terms of the previous expressions for shrinking neighborhoods $A$ around $x$,

$$
\lim \sup _{y \rightarrow x} \psi^{+}(y) \leq \psi^{+}(x), \quad \liminf _{y \rightarrow x} \psi^{-}(y) \geq \psi^{-}(x)
$$

\footnotetext{
${ }^{54}$ If $\alpha^{\prime}$ is the marginal of $\alpha$ on the variables $(\mu, x)$ - i.e., $\alpha^{\prime}=P \circ(\mu, i d)^{-1}$ - and $x \rightarrow \alpha^{\prime}(\cdot \mid x)$ is a decomposition of $\alpha^{\prime}$ conditional on $x$, then for $\alpha$-a.e. $x \in X, \psi^{+}(x)$ (resp. $\psi^{-}(x)$ ) is the supremum (resp. infimum) of the support of $\alpha^{\prime}(\cdot \mid x)$. The limits exist as the terms they are taken over are monotonic.
} 
where the limits are taken along $\operatorname{supp}\left(\alpha_{X}\right)$. These hold with equality when $x$ is not an atom of $\operatorname{supp}\left(\alpha_{X}\right)$; Indeed, if $x \in \operatorname{supp}\left(\alpha_{X}\right)$, then for each $\mu<\psi^{+}(x)$ and $\delta>0, \alpha(\{\theta \mid$ $\left.\left.\mu_{\theta} \geq \mu-\varepsilon\right\} \times(x-\delta, x+\delta)\right)>0$. If $x$ is not an atom of $\alpha_{X}$, then for some $y \in(x-\delta, x+\delta) \backslash\{x\}$, $\psi^{+}(y) \geq \mu$ by (14), and this was for any $\delta>0$ and any $\mu<\psi^{+}(x)$.

\section{Equilibrium Characterization}

We now discuss the proofs of Theorem 2 and Proposition 8.

We require only Assumptions 5, 6, and 7. Henceforth, fix an equilibrium $(p, \alpha)$. Recall $X=[0,1)$ or $X=[0,1]$. Recall also the pairs of functions $\psi^{+}, \psi^{-}$defined on the support of $\alpha_{X}$, the projection of the distribution $\alpha$ to [0,1], in Appendix B. Throughout we rely on the continuity of prices in equilibrium (Lemma 16 of Online Appendix $\mathrm{H}$ ).

\section{C.1 Coverage Increasing in Risk \& Separation of Types}

We now show that riskier types purchase strictly higher coverage (except possibly at $x=$ 0 ). Also, types of same riskiness purchase the same level. We begin with a weak version.

Lemma 2. It holds $\alpha$-a.s. that for each pair $\left(\theta_{2}, x_{2}\right),\left(\theta_{1}, x_{1}\right), x_{2}>x_{1}$ implies $\mu\left(\theta_{2}\right) \geq \mu\left(\theta_{1}\right)$. This is also true if $X^{\prime} \subseteq X$ is finite and $\alpha^{\prime}$ is a weak equilibrium of the economy $\left[\Theta \cup X^{\prime}, P \cup\right.$ $\left.\eta, X^{\prime}\right]$, where $X^{\prime}$ refers to behavioral types as well. ${ }^{55}$

Proof. Suppose not. Then there are open subsets $U, V$ of $\Omega \times[0,1]$ with $\alpha_{X}(U)>0$, $\alpha_{X}(V)>0$, and such that for each $\left(\theta_{1}, x_{1}\right) \in U,\left(\theta_{2}, x_{2}\right) \in V, x_{1}>x_{2}$ but $\mu_{1}=\mu\left(\theta_{1}\right)<\mu_{2}=$ $\mu\left(\theta_{2}\right)$. Fix such a pair. Then $p\left(x_{1}\right)$ must be above the indifference curve of type $\theta_{2}$ through $\left(x_{2}, p\left(x_{2}\right)\right)$, and $p\left(x_{2}\right)$ must be above the indifference curve of type $\theta_{1}$ through $\left(x_{1}, p\left(x_{1}\right)\right)$. Since the latter indifference curve is strictly flatter, this is impossible. Somewhat more formally, if $\phi_{j}$ denotes the indifference curve of $\mu_{j}$ through $\left(x_{j}, p_{j}\right)$ for $j=1,2$, then $\phi_{1}\left(x_{1}\right)=p\left(x_{1}\right) \leq \phi_{2}\left(x_{1}\right)$ and $\phi_{2}\left(x_{2}\right)=p\left(x_{2}\right) \leq \phi_{1}\left(x_{2}\right)$. However, $\phi_{j}^{\prime}(x)=w_{\mu_{j}}\left(x, \phi_{j}(x)\right)$ for $j=1,2$ a.e. and $\phi_{1}, \phi_{2}$ are absolutely continuous, and furthermore $w_{\mu_{1}}<w_{\mu_{2}}$ are continuous, hence $\phi_{1}\left(x^{\prime}\right)<\phi_{2}\left(x^{\prime}\right)$ in a neighborhood of $x^{\prime}$ of any point $x^{\prime} \in\left[x_{1}, x_{2}\right]$ at which $\phi_{1}\left(x^{\prime}\right)=\phi_{2}\left(x^{\prime}\right)$, contradiction. The same logic holds for weak equilibrium with behavioral types on a finite set of alternatives.

The following corollary shows that, if an agent with riskiness at least $\mu_{0}$ purchases a contract with coverage less than $x$, then the price of $x$ must be higher than the cost of type $\mu_{0}$ purchasing that contract.

\footnotetext{
${ }^{55}$ The conclusion then holds $\alpha^{\prime}(\cdot \mid \Theta)$-a.s., i.e., holds for types in $\Theta$, not behavioral types for whom $\mu$ is not defined.
} 
Corollary 5. For $x<y<z$, we have $\psi^{+}(x) \leq \psi^{-}(y) \leq \psi^{+}(y) \leq \psi^{-}(z)$. This is also true if $X^{\prime} \subseteq X$ is finite and $\alpha^{\prime}$ is a weak equilibrium of the economy $\left[\Theta \cup X^{\prime}, P \cup \eta, X^{\prime}\right]$.

Now, we show that there is no pooling of types: different levels of riskiness purchase different levels of coverage.

Lemma 3. $\psi^{-}=\psi^{+}$in $\operatorname{supp}\left(\alpha_{X}\right) \cap(0,1]$.

Proof. It follows from the corollary that for each $0 \leq y<x<z \leq 1, p(y) \leq x \cdot \psi^{-}(x) \leq$ $x \cdot \psi^{+}(x) \leq p(z)$, as $x \cdot \psi^{-}(x) \leq p(x) \leq x \cdot \psi^{+}(x) \alpha_{X}$ a.s. by (1). The continuity of prices gives the lemma for $x \in(0,1)$. For $x=1$, observe that if $\psi^{-}(1)<\psi^{+}(1)$, then since $\psi^{-}=\psi^{+}$in $X \cap(0,1), 1$ must be an atom of $\alpha_{X}$; then by the same continuity of prices (Lemma 16), $p(1) \leq \psi^{-}(1)$, and yet $p$ must be a strict average of $\psi^{-}(1), \psi^{+}(1)$, a contradiction.

Hence, denote $\psi=\psi^{-}=\psi^{+}$. By (1), we have

Lemma 4. $\alpha(\{\theta, x \mid p(x) \neq \mu(\theta) \cdot x\})=0$, and (equivalently), $p(z)=z \cdot \psi(z)$ for all $z \in \operatorname{supp}\left(\alpha_{X}\right)$.

Combined with the following lemma, price is strictly increasing in $\operatorname{supp}\left(\alpha_{X}\right) \cap(0,1]$.

Lemma 5. $\operatorname{supp}\left(P_{\mu}\right)=\operatorname{range}(\mu)$.

Proof. Follows from Assumption 7 together with the continuity of $\mu: \Theta \rightarrow \mathbb{R}_{+}$.

The proof of the follow proposition, using Assumption 7, is given in Online Appendix K.

Proposition 12. The following set is open:

$$
\left\{(\mu, p, x, q, y) \in \operatorname{supp}\left(P_{\mu}\right) \times \mathbb{R}_{+} \times[0,1] \times \mathbb{R}_{+} \times[0,1] \mid \mu \text { strictly prefers }(p, x) \text { over }(q, y)\right\}
$$

Lemma 6. $\psi$ is strictly increasing in $\operatorname{supp}\left(\alpha_{X}\right) \cap(0,1]$.

Proof. Suppose not; let $a<b$ be such that $\psi(a)=\psi(b)=\mu_{0}$. Corollary 5 then implies that $p(x)=\mu_{0} \cdot x$ for all $x \in[a, b]$. But since $w_{\mu_{0}}>\mu_{0}$ at all but full insurance, types with riskiness $\mu_{0}$ would all prefer $\left(b, p(b)=\mu_{0} \cdot b\right)$ over $\left(a, p(a)=\mu_{0} \cdot a\right)$. Hence by applying Proposition 12, types with riskiness near $\mu_{0}$ purchasing near $(a, p(a))$ would prefer to purchase $(b, p(b))$, and recalling the definition of $\psi(\cdot)$, gives the required result.

\section{C.2 Coverage Continuous and Increasing in Risk}

Corollary 6. There is a mapping $\sigma: \operatorname{supp}\left(P_{\mu}\right) \rightarrow[0,1]$, strictly increasing and continuous on $\operatorname{supp}\left(P_{\mu}\right) \backslash \sigma^{-1}(\{0\})$, s.t. $\alpha\{(\theta, x) \mid x=\sigma(\mu(\theta))\}=1$. 
Proof. Let $W \subseteq \Theta$ such that $\alpha(\{\theta \in W\} \Delta\{x>0\})=1$, i.e., those types which choose positive coverage. Let $\sigma=\psi^{-1} . \sigma$ is well-defined $P_{\mu}$-a.e. on $\operatorname{supp}\left(\left(P_{\mu}(\cdot \mid W)\right)\right.$, and by the previous results in strictly monotonic. Extend $\sigma$ to $\operatorname{supp}\left(P_{\mu}(\cdot \mid \Theta \backslash W)\right)$ by 0 ; by the previous results, this is well-defined ( $\alpha$-a.s., any types $\theta$ s.t. $\mu(\theta) \in \operatorname{supp}\left(\left(P_{\mu}(\cdot \mid W)\right) \cap\right.$ $\operatorname{supp}\left(P_{\mu}(\cdot \mid \Theta \backslash W)\right)$ choose 0 coverage, i.e., are not in $W$.)

\section{C.3 Full Insurance is in the Support}

Lemma 7. The supremum of the support of $\alpha_{X}$ is full insurance.

Proof. Suppose $x^{*}<1$ is the supremum of the support of $\alpha_{X}$. By Corollary 5 (applied to a sequence of weak equilibria on finite grids which witness the equilibrium), for each $x>x^{*}$ and each $\mu \in \operatorname{supp}\left(P_{\mu}\right), p(x) \geq \mu x$. Hence, $P_{\mu}$ is compactly supported. Denote $\bar{\mu}=\max \operatorname{supp}\left(P_{\mu}\right)$. Hence, $p(x) \leq \bar{\mu} x$ for $x \geq x^{*}$ (price per unit cannot be more than maximum costliness) and since $p$ is continuous and prices are actuarily fair, $p\left(x^{*}\right)=\bar{\mu} x^{*}$; so $p(x)=\bar{\mu} x$ in $\left[x^{*}, 1\right]$. A contradiction follows as in the proof of Lemma 6 .

The proof is illustrated in Figure 7 below in Online Appendix K.

\section{C.4 Lipschitz-Type Property}

The fact that $L$-Lipschitz-ness of price in $\left[0, x_{0}\right]$ if $w_{\theta} \leq L$ in $\left\{x \leq x_{0}, p \leq p\left(x_{0}\right)\right\}$ for a.e. types purchasing coverage up to $x_{0}$ follows along the lines of Part 3 of Proposition 1 of AG, so we omit a complete proof. Essentially, the restriction of the economy to those types that choose coverage up to $x_{0}$ satisfies the framework and Lipschitz-ness conditions of that paper. As for the conclusion that $p(\cdot)$ is Lipschitz (for some constant) in $\left[0, x_{0}\right]$ without assuming a bound on $w_{\theta}$, observe that since the coverage function is increasing in $\mu$, and the willingness to pay in increasing is risk (Assumption 6). Take any $\theta$ with $\sigma(\mu(\theta))>x_{0}$ and set $L=\max \left\{w_{\theta}(x, p) \mid x \leq x_{0}, p \leq p\left(x_{0}\right)\right\}$. Then, $w_{\theta} \leq L$ in $\left\{x \leq x_{0}, p \leq p\left(x_{0}\right)\right\}$ for a.e. all types purchasing coverage up to $x_{0}$.

\section{C.5 Price as Approximate Upper Envelope}

We prove the upper envelope property of equilibrium, expressed in 9. Inequality $(\geq)$ holds: otherwise there would be a positive mass of types with $\sigma(\mu(\theta))>x$ and $\eta(\theta, x)>$ $p(x)$, so they would prefer $(x, p(x))$ over their purchased contract - a contradiction.

Conversely, suppose for some $x^{*}<y^{*}$ and $\delta>0$, we have $\alpha_{X}\left(\left(x^{*}, y^{*}\right]\right)>0$ but $p\left(x^{*}\right)>\zeta\left(x^{*}, y^{*}\right)$. Then, there is a gap $\left(x^{*}, z^{*}\right)$ in with $\alpha_{X}\left(\left(x^{*}, z^{*}\right)\right)=0$ but $z^{*} \in \operatorname{supp}\left(\alpha_{X}\right)$. Similarly, there is a gap $P_{\mu}\left(\mu_{*}, \mu^{*}\right)=0, \sigma\left(\mu^{*}\right)=z^{*}$. We contend $\mu^{*}$ strictly prefers 
$B:=\left(z^{*}, p\left(z^{*}\right)\right)$ to $A:=\left(x^{*}, p\left(x^{*}\right)\right)$. If he weakly preferred $A$ to $B$, he would strictly pre-

fer $A^{\prime}=\left(x^{*}, \frac{p\left(x^{*}\right)+\zeta\left(x^{*}, y^{*}\right)}{2}\right)$ to $B$; but then by Proposition 12, types near $\mu^{*}$ would strictly prefer $A^{\prime}$ to contracts near $z^{*}$, contradicting $\zeta\left(x^{*}, y^{*}\right)<\frac{p\left(x^{*}\right)+\zeta\left(x^{*}, y^{*}\right)}{2}$. Again by Proposition 12, type $\mu^{*}$ (and hence, since $w_{\mu}$ is increasing in $\mu$, all types with riskiness $\geq \mu^{*}$ ) prefer all contracts in some neighborhood $V$ of $B$ to all those in some neighborhood $U$ of $A$. Letting $\left(p^{n}, \alpha^{n}\right)_{n=1}^{\infty}$ be weak equilibria converging to $(p, \alpha)$ in the required sense; the following lemma (proved in Online Appendix K) gives a contradiction, as for $n$ large enough, contracts in $V$ are available:

Lemma 8. For each $\varepsilon, \delta>0$, for $n$ large enough, under $\alpha_{n}$ a positive measure of types with riskiness in $\left[\mu^{*}, \mu^{*}+\delta\right)$ purchase coverage in $\left(x^{*}-\varepsilon, x^{*}+\varepsilon\right)$.

\section{C.6 No Purchasing of 0 Coverage}

If $P$ is discrete, with atoms $\mu_{1}<\mu_{2}<\cdots$, then for some $n, \sigma\left(\mu_{n}\right)>0$, and by backward induction using the 'upper-envelope' property of prices, $\sigma\left(\mu_{k}\right)>0$ for all $k<n$ as well. The continuous case is sketched in Online Appendix K.

\section{Unbounded Price}

We prove Proposition 10. Throughout this section, fix $\bar{\delta}>0$ for which Assumption 8 holds. Suppose that $(p, \alpha)$ constitute an AG-equilibrium. We associate to the distribution $\alpha$ a pair $\psi^{+}, \psi^{-}$as defined in Section B. We proceed in several steps.

\section{D.1 Comparisons of Utilities}

Assumption 4 implies Lemma 9 below, which is later used to obtain upper and lower bounds on the slopes of the price in equilibrium.

Lemma 9. Under Assumption 4, in any equilibrium (or weak equilibrium) $(p, \alpha)$, if $1 \geq$ $x_{2} \geq x_{1} \geq \delta \geq 0$, then for any given type $\theta \in \Theta$ :

$$
\begin{aligned}
& u_{\theta}\left(p_{2}, x_{2}\right) \geq u_{\theta}\left(p_{1}, x_{1}\right) \Rightarrow \frac{p_{2}-p_{1}}{x_{2}-x_{1}} \leq \mu_{\theta}+\bar{\nu}_{\delta}(\theta) \cdot\left(1-\frac{x_{1}+x_{2}}{2}\right) \\
& u_{\theta}\left(p_{2}, x_{2}\right) \leq u_{\theta}\left(p_{1}, x_{1}\right) \Rightarrow \frac{p_{2}-p_{1}}{x_{2}-x_{1}} \geq \mu_{\theta}+\underline{\nu}_{\delta}(\theta) \cdot\left(1-\frac{x_{1}+x_{2}}{2}\right)
\end{aligned}
$$


Proof. By Assumption 4, $u_{\theta}(x, p)=g_{\theta}(x)-p$ and $w_{\theta}(1)=\mu_{\theta}+\left.\frac{\partial g_{\theta}}{\partial x}\right|_{x=1}=\mu_{\theta}$, so $\frac{\partial g_{\theta}}{\partial x}(s)=$ $\mu_{\theta}-\int_{s}^{1} \frac{\partial^{2} g_{\theta}}{\partial^{2} x} d x$. Now, for any $0 \leq x_{1}<x_{2} \leq 1$,

$$
u_{\theta}\left(x_{2, p_{2}}\right)-u_{\theta}\left(x_{1}, p_{1}\right)=p_{1}-p_{2}+\int_{x_{1}}^{x_{2}} \frac{\partial g_{\theta}}{\partial x} d x=p_{1}-p_{2}+\mu_{\theta}\left(x_{2}-x_{1}\right)+\int_{x_{1}}^{x_{2}} \int_{s}^{1}\left(-\frac{\partial^{2} g_{\theta}}{\partial^{2} x}\right) d x .
$$

Observing that

$$
\int_{x_{1}}^{x_{2}} \int_{s}^{1} 1 d x=\frac{1}{2}\left(1-x_{1}\right)^{2}-\frac{1}{2}\left(1-x_{2}\right)^{2}=\left(x_{2}-x_{1}\right)\left(1-\frac{x_{1}+x_{2}}{2}\right) .
$$

and that for $x_{2}>x_{1} \geq \delta, \underline{\nu}_{\delta}(\theta) \leq-\frac{\partial^{2} g_{\theta}}{\partial^{2} x} \leq \bar{\nu}_{\delta}(\theta)$, completes the proof.

\section{D.2 Price is Locally Lipschitz in Equilibrium}

The results of this section are where we use the assumption, made for convenience, that $\bar{\rho}_{\bar{\delta}}(\cdot)$ is right-continuous. Our first auxiliary results establish bounds on prices in a weak equilibrium (hence also in equilibrium). ${ }^{56}$

Lemma 10. Assume $(p, \alpha)$ is an equilibrium or weak equilibrium s.t. $p$ is continuous on supp $\left(\alpha_{X}\right) .{ }^{57}$ Let $0<x_{1}<x_{2}$ be two points in supp $\left(\alpha_{X}\right)$. Then,

$$
\begin{aligned}
\frac{p\left(x_{1}\right)}{x_{1}} & \leq \psi^{+}\left(x_{1}\right) \leq \frac{p\left(x_{2}\right)-p\left(x_{1}\right)}{x_{2}-x_{1}} \\
& \leq \psi_{\text {if } x_{1}>1-\bar{\delta}} \psi^{-}\left(x_{2}\right)+\left(1-\frac{x_{1}+x_{2}}{2}\right) \bar{\rho}_{\bar{\delta}}\left(\psi^{-}\left(x_{2}\right)\right) \leq \frac{p\left(x_{2}\right)}{x_{2}}+\left(1-\frac{x_{1}+x_{2}}{2}\right) \bar{\rho}_{\bar{\delta}}\left(\frac{p\left(x_{2}\right)}{x_{2}}\right)
\end{aligned}
$$

In particular, $\psi^{+}(x)$ must be finite for each $x \in \operatorname{supp}\left(\alpha_{X}\right)$ with $x<1$.

Proof. The break-even condition $\left(p(x)=\mathbb{E}_{\alpha}[c \mid x], \alpha\right.$-a.e. $\left.x\right)$ requires that for $\alpha_{X}$-a.e. $x>$ 0 in $\operatorname{supp}\left(\alpha_{X}\right), \psi^{-}(x) \leq \frac{p(x)}{x} \leq \psi^{+}(x)$. This, together with the monotonicity of $\bar{\rho}_{\bar{\delta}}$, implies the first and last inequalities. The third inequality follows primarily from Lemma 9 and the fact that $0 \leq \underline{\nu} \bar{\delta} \leq \bar{\nu}_{\bar{\delta}} \leq \bar{\rho}_{\bar{\delta}}$; details in Online Appendix K.

The second auxiliary result shows the outer-most bounds hold for equilibria everywhere (not just on the support of $\alpha_{X}$ ).

Lemma 11. Assume $(p, \alpha)$ is an equilibrium. If $0<1-\bar{\delta}<x_{1}<x_{2}$ with $p\left(x_{2}\right)>0\left(x_{1}, x_{2}\right.$ are not necessarily in the support of $\left.\alpha_{X}\right)$, then

$$
\frac{p\left(x_{1}\right)}{x_{1}} \leq \frac{p\left(x_{2}\right)-p\left(x_{1}\right)}{x_{2}-x_{1}} \leq \frac{p\left(x_{2}\right)}{x_{2}}+\left(1-\frac{x_{1}+x_{2}}{2}\right) \bar{\rho}_{\bar{\delta}}\left(\frac{p\left(x_{2}\right)}{x_{2}}\right)
$$

\footnotetext{
${ }^{56}$ We defined "weak equilibrium" in Section 2.

${ }^{57} \mathrm{In}$ particular, this is true if $\operatorname{supp}\left(\alpha_{X}\right)$ is finite.
} 
In particular, $p$ is non-decreasing.

Proof. If $p\left(x_{1}\right)=0$, the first inequality is trivial, so assume $p\left(x_{1}\right)>0$; the result follows by applying Lemma 10 to each of a sequence of weak equilibria which converge to $(p, \alpha)$; for details see Online Appendix K.

\section{D.3 Full insurance is Not a Heterogeneous Atom}

We show full insurance $(x=1)$ cannot be an atom of $\alpha_{X}$ purchased by multiple types if $\mu_{\theta}$ is not essentially bounded w.r.t. $P$. This proposition is only relevant when $1 \in X$.

Lemma 12. If $x=1$ is an atom of $\alpha_{X}$, it must be that $\alpha(\cdot \mid\{x=1\})$ is concentrated on one riskiness, i.e., there must be $\tilde{\mu} \in \mathbb{R}_{+}$s.t. $\alpha(\mu(\theta)=\tilde{\mu} \mid x=1)=1$.

Proof. Suppose, by way of contradiction, that $x=1$ is an atom of $\alpha_{X}$ but not concentrated on a single riskiness. Then, the break-even condition (1) requires that there are some types buying $x=1$ who are less risky than the average buyers of that contract, with $p(1)$ being determined by these average buyers. Define $\mu^{\star}=\psi^{-}(1)$ and $p^{\star}:=p(1)$; then $\mu^{\star}<E_{\alpha}[\mu \mid x=1]=p(1)=p^{\star}$. Lemma 10 implies that, for any $x \geq 1-\bar{\delta}$,

$$
\frac{p(x)}{x} \leq \frac{p^{\star}-p(x)}{1-x} \leq \mu^{\star}+\frac{1}{2} \bar{\rho}_{\bar{\delta}}\left(\mu^{\star}\right)(1-x) .
$$

In turn, this implies

$$
p^{\star} x \geq p(x) \geq p^{\star}-\mu^{\star}(1-x)-\frac{1}{2} \bar{\rho}_{\bar{\delta}}\left(\mu^{\star}\right)(1-x)^{2} .
$$

It then follows that $\frac{1}{2} \bar{\rho}_{\bar{\delta}}\left(\mu^{\star}\right)(1-x) \geq p^{\star}-\mu^{\star}$. However, this last condition cannot hold for $x$ close enough to 1 since $p^{\star}>\mu^{\star}$, a contradiction.

Figure 5 illustrates graphically the idea of the proof of Lemma 12.

\section{D.4 Proposition 10}

Suppose not. Denote $p^{\star}=\lim _{x \rightarrow 1} p(x)<\infty$. Fix some $0<\bar{\delta} \leq \delta<1$. Recall that $p^{\star}=p(1)$. Then, from Lemma 11 and the facts that $p$ and $\bar{\rho}_{\bar{\delta}}$ are non-decreasing, for all $1-\delta \leq x_{1}<x_{2}$ in $X$,

$$
\psi^{+}\left(x_{1}\right) \leq \frac{p\left(x_{2}\right)-p\left(x_{1}\right)}{x_{2}-x_{1}} \leq \frac{p\left(x_{2}\right)}{x_{2}}+\bar{\nu}\left(\frac{p\left(x_{2}\right)}{x_{2}}\right) \leq M:=\frac{p^{*}}{1-\delta}+\bar{\rho}_{\bar{\delta}}\left(\frac{p^{*}}{1-\delta}\right),
$$

while for $x_{1} \leq 1-\delta$ in $X$,

$$
\psi^{+}\left(x_{1}\right) \leq \lim _{x_{2} \rightarrow 1^{-}} \frac{p\left(x_{2}\right)-p\left(x_{1}\right)}{x_{2}-x_{1}}=\frac{p^{*}-p\left(x_{1}\right)}{1-x_{1}} \leq \frac{p^{*}}{\delta}
$$


Contradiction to Atom at Full Insurance:

$\left(p^{*}, 1\right)$ Should Be Above Red Dashed Line

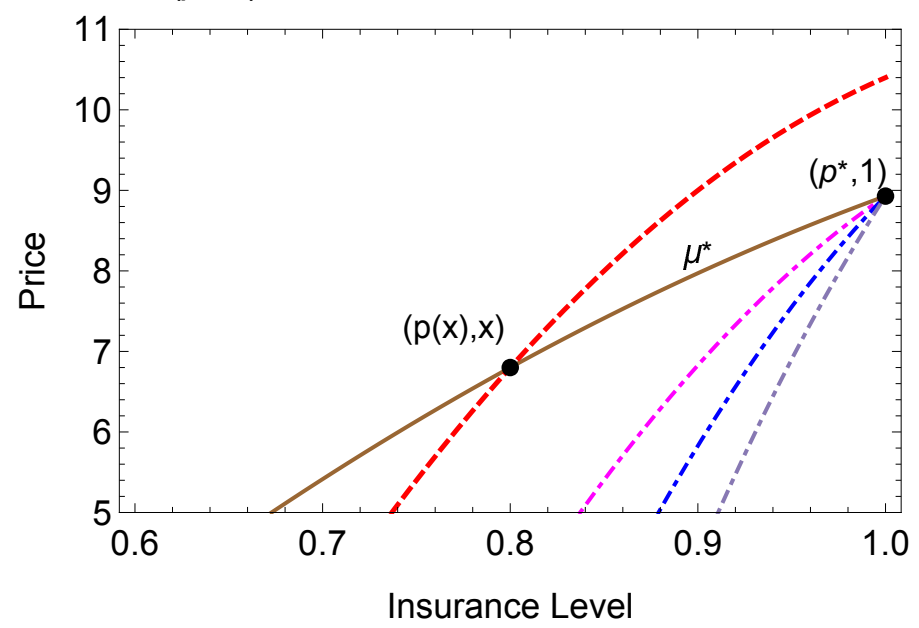

Riskiness of Least Risky Full a Insurance Purchaser $=\mu^{*}<p^{*}$

Riskiness of Agent Purchasing $(p(x), x) \geq \frac{p(x)}{x}>\mu^{*}$

Figure 5: Proof of Lemma 12.

Hence, $\sup _{x<1} \psi^{+}(x) \leq \max \left[M, \frac{p^{*}}{\delta}\right]$. Since full insurance cannot be an atom with unbounded support of riskiness of $\alpha$, by Lemma 12, the projection of $\alpha(\cdot \mid[0,1))$ to riskiness $\mu$ is not compactly supported. Applying (14) with $A=X \backslash\{1\}$ gives $\sup _{x<1} \psi^{+}(x)=\infty$.

\section{D.5 Non-Existence: Premium Bounded by Linear Function of Risk}

Fix $\bar{\delta}>0$ for which Assumption 8 holds. Let $C, D$ be such that $\bar{\nu}_{\delta}(\theta) \leq C \mu(\theta)+D P$-a.s.. Let $x_{n} \rightarrow 1$ strictly monotonically in $X$. WLOG, $x_{1}>1-\bar{\delta}$. Denote $B=\max \left[\frac{1+C}{x_{1}}, D\right]$ and $p_{n}=p\left(x_{n}\right)$. Then, $\bar{\rho}_{\bar{\delta}}\left(\frac{p_{n+1}}{x_{n+1}}\right) \leq C \frac{p_{n+1}}{x_{n+1}}+D$, so by Lemma 11 ,

$$
\frac{p_{n+1}-p_{n}}{x_{n+1}-x_{n}} \leq \frac{p_{n+1}}{x_{n+1}}+\left(1-x_{n}\right) \bar{\rho}_{\bar{\delta}}\left(\frac{p_{n+1}}{x_{n+1}}\right) \leq \frac{p_{n+1}}{x_{n+1}}+C \frac{p_{n+1}}{x_{n+1}}+D \leq B\left(p_{n+1}+1\right)
$$

Denote $q_{n}=p_{n}+1$ and $\delta_{n}=B\left(x_{n+1}-x_{n}\right)$; w.l.o.g. $\delta_{n}<1$ for all $n$, and hence

$$
q_{n+1}-q_{n} \leq \delta_{n} q_{n+1} \Longrightarrow q_{n+1} \leq \frac{q_{n}}{1-\delta_{n}} \Longrightarrow q_{n} \leq q_{1} \cdot \prod_{j<n} \frac{1}{1-\delta_{j}}
$$

by induction. Then, $\sum \delta_{n}=\sum B\left(x_{n+1}-x_{n}\right)=B\left(1-x_{1}\right)<\infty$ which implies $\prod_{j<\infty} \frac{1}{1-\delta_{j}}<$ $\infty$. This, together with the monotonicity of $p(\cdot)$, shows that $\sup _{x \in X} p(x)=\lim _{n \rightarrow \infty} p_{n}<\infty$ which contradicts Proposition 11. 


\section{E Simple Insurance: Continuous Types}

\section{E.1 Proposition 13}

Proposition 13. If $P_{\mu}$, conditional on some interval $I=(\underline{\mu}, \bar{\mu})$, has full support with a.e. strictly positive density w.r.t. the Lebesgue measure, and $(p, \alpha)$ is an equilibrium with associated coverage function $\sigma$ with $\sigma(\underline{\mu})>0$, then

$$
\int_{\underline{\mu}}^{\bar{\mu}} \frac{1}{\nu(\mu)} d \mu=\int_{\sigma(\underline{\mu})}^{\sigma(\bar{\mu})} \frac{g^{\prime}(x)}{x} d x
$$

Proof. Let $(p, \alpha)$ be an equilibrium, with associated $\sigma: I \rightarrow[0,1]$. Denoting $J=[\sigma(\underline{\mu}), \sigma(\bar{\mu})]$, $\alpha_{J}$ is equivalent (i.e., has the same null sets) as the Lebesgue measure in $J$ (see Proposition 15 of Online Appendix K). Given the differentiability of the price function Lebesgue a.s. in $J$ (equivalently, $\alpha_{J}$-a.s.), utility maximization implies

$$
\left.\frac{\partial}{\partial x}\left(u_{\mu}(x, p(x))\right)\right|_{x=\sigma(\mu)}=0 \Leftrightarrow \frac{\partial u_{\tau(x)}}{\partial x}(x, p(x))+\frac{\partial u_{\tau(x)}}{\partial p}(x, p(x)) \cdot p^{\prime}(x)=0, \text { a.e. } x \in J
$$

where $\tau(x)=\frac{p(x)}{x}=\sigma^{-1}(x)$. Explicitly: For this to hold at some $x$ we need two conditions: $p$ to be differentiable at $x$, and for the type with riskiness $\mu=\sigma^{-1}(x)$ to be a utility maximizer. Both of these properties hold Lebesgue a.s. in $J$, equivalently, $\alpha_{J}$-a.s. Hence,

$$
p^{\prime}(x)=-\frac{\frac{\partial u_{\tau(x)}}{\partial x}(x, p(x))}{\frac{\partial u_{\tau(x)}}{\partial p}(x, p(x))}=\frac{p(x)}{x}+g^{\prime}(x) \cdot \nu\left(\frac{p(x)}{x}\right), \text { a.e. } x \in J
$$

Denote $\tau=\sigma^{-1}$ on $J$. The equilibrium property of actuarily fairness (and continuity of prices and $\tau$ ) shows that $p(x)=\tau(x) \cdot x, \forall x \in J$ and hence $\sigma^{-1}$ is differentiable a.e. and

$$
p^{\prime}(x)=\tau(x)+x \cdot \tau^{\prime}(x), \text { a.e } . x \in J .
$$

Rewriting (20) gives

$$
\tau(x)+g^{\prime}(x) v(\tau(x))-p^{\prime}(x)=0 \text {, a.e. . } x \in J .
$$

Summing these gives

$$
g^{\prime}(x) \cdot \nu(\tau(x))=x \cdot \tau^{\prime}(x) \Longrightarrow \frac{g^{\prime}(x)}{x}=\frac{1}{\nu(\tau(x))} \tau^{\prime}(x), \text { a.e. . } x \in J
$$


and hence, by change of variable (note that $\tau$ is strictly increasing),

$$
\int_{\sigma(\underline{\mu})}^{\sigma(\bar{\mu})} \frac{g^{\prime}(x)}{x} d x=\int_{\sigma(\underline{\mu})}^{\sigma(\bar{\mu})} \frac{1}{\nu(\tau(x))} \tau^{\prime}(x) d x=\int_{\underline{\mu}}^{\bar{\mu}} \frac{1}{\nu(\mu)} d \mu
$$

as required. Note that we have made use of a change of variable formula for functions which are differentiable a.e., see for instance Rudin [1987, Theorem 7.26].

\section{E.2 Corollary 2}

Follows from Proposition 13. From Proposition 8, under these assumptions, we have $\sigma(\bar{\mu})=1$. The right-hand side defines $\sigma$ uniquely as $g^{\prime}>0$ except possibly at 1 . The last conclusion follows since $g^{\prime}(0)>0$ so $\int_{0}^{1} \frac{g^{\prime}(x)}{x} d x=\infty$.

\section{E.3 Corollary 4}

We prove the characterization part first: WLOG, $\left(\bar{\mu}_{n}\right)_{n}$ is increasing. Define the function $\sigma$ using (5). Since $g^{\prime}>0$ in $(0,1)$ and $\int_{0}^{1} \frac{g^{\prime}(x)}{x} d x=\infty>\int_{\mu}^{\infty} \frac{1}{\nu(\mu)} d \mu$ for all $\mu>\underline{\mu}$, this is well-defined, continuous, and its image is an interval. Let $x_{\min }=e s s-\inf (\sigma(\mu(\theta)))$, and $x_{\text {min }}^{n}=$ ess $-\inf \left(\sigma^{n}(\mu(\theta))\right) .{ }^{58}$ Define $\tau=\sigma^{-1}:\left[x_{\text {min }}, 1\right) \rightarrow \mathbb{R}, p(x)=x \cdot \tau(x)$ for $x \in[\sigma(\underline{\mu}), 1)$, and extend $p$ in $\left[0, x_{\text {min }}\right)$ in the following way: For each $x \in\left[0, x_{\text {min }}\right)$, set

$$
p(x)=\max \left[0, e s s-\sup _{\theta} \phi(\theta, x)\right] \quad \text { where } \quad \phi(\theta, x)=p\left(\sigma(\mu(\theta))-\int_{x}^{\sigma(\mu(\theta))} w_{\theta}(t) d t\right.
$$

where $\phi(\theta, \cdot)$ is the indifference curve of type $\theta$ through his purchased contract $(\sigma(\mu, \theta), p(\sigma(\mu(\theta))$. Finally, define the application $\alpha$ for $\tilde{\Theta} \subseteq \Theta$ and $a<b$ by,

$$
\alpha(\theta \in \tilde{\Theta}, x \in(a, b))=P(\theta \in \tilde{\Theta}, \mu(\theta) \in(\tau(a), \tau(b)))
$$

By (3), we obtain

$$
\int_{\mu}^{\bar{\mu}_{n}} \frac{1}{\nu(\mu)} d \mu=\int_{\sigma^{n}(\mu)}^{1} \frac{g^{\prime}(x)}{x} d x .
$$

Comparing with (5), it follows that $\sigma^{n} \rightarrow \sigma$ point-wise, and in particular $x_{\min }^{n} \rightarrow x_{\min }$, and $\left(x_{\min }^{n}\right)$ is monotonically decreasing. Letting $\tau_{n}=\sigma_{n}^{-1}:\left[x_{\min }, 1\right)$, we have $\tau_{n} \rightarrow \tau$ point-wise. Therefore, $p^{n} \rightarrow p$ point-wise on $\left[x_{\text {min }}, 1\right]$, as $p^{n}(x)=x \cdot \tau^{n}(x)$ on $\left[x_{\min }^{n}, 1\right]$. Let $x^{\prime}<x_{\min }$; for large enough $n, x<x_{\min }^{n}$. Recall from Proposition 8 ,

$$
p^{n}(x)=\max \left[0, e s s-\sup _{\theta} \phi^{n}(\theta, x)\right] \quad \text { where } \quad \phi^{n}(\theta, x)=p\left(\sigma^{n}(\mu(\theta))-\int_{x}^{\sigma^{n}(\mu(\theta))} w_{\theta}(t) d t\right.
$$

\footnotetext{
${ }^{58}$ The essential infimum is defined similar to the essential supremum.
} 
$\phi^{n}(\theta, \cdot)$ is the indifference curve of type $\theta$ through his chosen contract under $\left(p^{n}, \sigma^{n}\right)$. Notice for $n>k, \overline{\mu_{n}} \geq \bar{\mu}_{k}$, so $\sigma_{n} \leq \sigma_{k}$, so $\tau_{n} \geq \tau_{k}$; furthermore, $\phi^{n} \geq \phi^{k}$ (the indifference curve of each type moves to the left, alongside the actuarily fair price for that type, a line whose slope is flatter than that types indifference curve), so $p_{n} \geq p_{k}$. Hence $p^{n} \rightarrow p$ point-wise in $\left[0, x_{\text {min }}\right]$ as well, as $\left(\phi^{n}(\theta, \cdot)\right)$ increasing in $n .{ }^{59}$ Hence, $p_{n} \rightarrow p$ point-wise. Since $n>k \rightarrow p_{n} \geq p_{k}$, Dini's theorem implies that $p_{n} \rightarrow p$ uniformly in every compact subset of $[0,1)$.

Finally, for any continuous bounded $f:[\underline{\mu}, \infty) \times[0,1] \rightarrow \infty$,

$$
\int f(\mu, x) \cdot d \alpha=\int f(\mu, \sigma(\mu)) d P_{\mu}=\lim _{n \rightarrow \infty} \int f\left(\mu, \sigma_{n}(\mu)\right) d P_{\mu}=\lim \int f(\mu, x) \cdot d \alpha_{n}
$$

by the bounded convergence theorem, and hence $\alpha_{n} \rightarrow \alpha$ weakly.

We now prove the first point of Corollary 4. Existence now follows from the approximation result Proposition 1. Uniqueness can we proved as follows. Given any equilibrium with associated choice function $\sigma$, for each $\bar{\mu}>\underline{\mu}$, (19) holds. Take $\bar{\mu} \rightarrow \infty$. Since full coverage is in the support of the equilibrium and $\sigma$ is increasing, $\lim _{\mu \rightarrow \infty} \sigma(\mu)=1$. The last part of the proposition follows again as $\int_{0}^{1} \frac{g^{\prime}(x)}{x} d x=\infty$.

\section{E.4 Corollary 3}

For the non-existence part: We can find intervals $I=\left[a_{n}, b_{n}\right]$ with $a_{n}, b_{n} \rightarrow \infty$ for which $\int_{a_{n}}^{b_{n}} \frac{1}{\nu(\mu)} d \mu \rightarrow \infty$ but since $\sigma\left(a_{n}\right), \sigma\left(b_{n}\right) \rightarrow 1, \int_{\sigma\left(a_{n}\right)}^{\sigma\left(b_{n}\right)} \frac{g^{\prime}(x)}{x} d x \rightarrow 0$, which contradicts (19).

For the price divergence (equivalently, purchasing converging to 0): fixing $\mu \in \mu(\Theta)$,

$$
\int_{\mu}^{\bar{\mu}_{n}} \frac{1}{\nu\left(\mu^{\prime}\right)} d \mu^{\prime}=\int_{\sigma^{n}(\mu)}^{1} \frac{g^{\prime}(x)}{x} d x \Longrightarrow \int_{\mu}^{\infty} \frac{1}{\nu\left(\mu^{\prime}\right)} d \mu^{\prime}=\infty=\lim _{n \rightarrow \infty} \int_{\sigma^{n}(\mu)}^{1} \frac{g^{\prime}(x)}{x} d x
$$

However,

$$
\int_{\sigma^{n}(\mu)}^{1} \frac{g^{\prime}(x)}{x} d x \leq \max _{[0,1]} g^{\prime} \cdot \int_{\sigma^{n}(\mu)}^{1} \frac{1}{x} d x=\ln \left(\sigma^{n}(\mu)\right) \cdot \max _{[0,1]} g^{\prime}
$$

For this to converge to $\infty$, we must have $\sigma^{n}(\mu) \rightarrow 0$.

\section{F Simple Insurance: Discrete Types}

For each $k \in \mathbb{N}$ and each coverage $u \in[0,1]$, let $v=\phi_{k}(u)$ denote the unique coverage $v<$ $u$ such that type $\theta_{k+1}=\left(\mu_{k+1}, \nu_{k+1}\right)$ is indifferent between contracts $(p, x)=\left(\mu_{k+1} \cdot u, u\right)$ and $(p, x)=\left(\mu_{k} \cdot v, v\right)$, where the second contract is actuarily fair for type $\theta_{k}$. Such unique $v$ exists as $w_{\theta_{k+1}}(x)>\mu_{k+1}>\mu_{k}$ for all $x \in(0,1)$. Clearly $z>\phi_{k}(z)$ for $z \in(0,1]$.

\footnotetext{
${ }^{59}$ Lemma: Let $\left(f_{n}\right)$ be a sequence of bounded Borel functions on a measurable space, $f_{1} \leq f_{2} \leq f_{3} \leq \ldots$ which converge point-wise to $f$. Then ess $-\sup \left(f_{n}\right) \rightarrow e s s-\sup (f)$.
} 
Lemma 13. $0<\phi_{k}^{\prime} \leq 1$ in $(0,1)$. Therefore, $\phi_{k}$ is strictly increasing (in particular, $\phi_{k}(u)=0$ if and only if $u=0$ ). Moreover, $\phi_{k}$ is non-expansive (i.e., 1-Lipschitz).

Proof. The definition of $\phi_{k}(\cdot)$ requires that, for each $z \in(0,1]$, type $k+1$ is indifferent between $\left(\mu_{k+1} \cdot z, z\right)$ and $\left(\mu_{k} \cdot \phi_{k}(z), \phi_{k}(z)\right)$. This implies

$$
\mu_{k+1} \cdot \phi_{k}(z)+g\left(\phi_{k}(z)\right) \cdot \nu_{k+1}-\mu_{k} \cdot \phi_{k}(z)=\mu_{k+1} \cdot z+g(z) \cdot \nu_{k+1}-\mu_{k+1} \cdot z=g(z) \cdot \nu_{k+1}
$$

where $\nu_{k+1}=\nu\left(\mu_{k+1}\right) \cdot g$ is differentiable with $g^{\prime}>0$ in $(0,1)$, so $\phi_{k}$ is differentiable with,

$$
\begin{gathered}
\phi_{k}^{\prime}(z) \cdot\left[\mu_{k+1}-\mu_{k}+g^{\prime}\left(\phi_{k}(z)\right) \cdot \nu_{k+1}\right]=g^{\prime}(z) \cdot \nu_{k+1} \Longrightarrow \\
\phi_{k}^{\prime}(z)=\frac{g^{\prime}(z) \cdot \nu_{k+1}}{\mu_{k+1}-\mu_{k}+g^{\prime}\left(\phi_{k}(z)\right) \cdot \nu_{k+1}} \leq \frac{g^{\prime}(z) \cdot \nu_{k+1}}{g^{\prime}\left(\phi_{k}(z)\right) \cdot \nu_{k+1}}=\frac{g^{\prime}(z)}{g^{\prime}\left(\phi_{k}(z)\right)} .
\end{gathered}
$$

Since $g$ is concave, $g^{\prime}$ is decreasing. Since $\phi_{k}(z)<z$, so $g^{\prime}\left(\phi_{k}(z)\right)>g^{\prime}(z)$, so $0<\phi_{k}^{\prime} \leq 1$

We now prove Proposition 4. First we show uniqueness: suppose an equilibrium in which type $\left(\mu_{k}, \nu_{k}\right)$ purchases coverage $x_{k}=\sigma\left(\mu_{k}\right)$ then $x_{1}<x_{2}<\cdots$ By the equilibrium properties, $\sigma\left(\mu_{k}\right)=\phi_{k}\left(\sigma\left(\mu_{k+1}\right)\right)$, i.e., $x_{k}=\phi_{k}\left(x_{k+1}\right)$. Inductively, for any $n>k$,

$$
x_{k}:=\phi_{k}\left(\phi_{k+2}\left(\cdots\left(\phi_{n-1}\left(x_{n}\right)\right) \cdots\right)\right)=\lim _{n \rightarrow \infty} \phi_{k}\left(\phi_{k+2}\left(\cdots\left(\phi_{n}(1)\right) \cdots\right)\right)
$$

where the latter equality follows from Lemma 13, which shows that $\phi_{k}$ is non-expansive, which defines $x_{k}$ uniquely (since each $\phi_{k}$ is monotonic, the expression the limit is taken over is decreasing with $k$ but non-negative, so the limit exists).

We now present a slightly heuristic (but easily formalized) argument, for why (7) guarantees existence of equilibrium, by choosing that we have a well-defined sequence of positive elements $\left(x_{k}\right)_{k=1}^{\infty}$. (It suffices to show it gives some positive $x_{K}$, and then $x_{1}, \ldots, x_{K-1}$ are defined from it recursively using $\phi_{1}, \ldots, \phi_{K-1}$.) Fix $\delta$ small and a range of values, and suppose $z, \phi_{k}(z) \approx 1-\delta$; denoting $G=-g^{\prime \prime}(1)>0$, since $g^{\prime}(1)=0$, a second order expansion around $x=1$ gives

$$
\begin{aligned}
g(z)-g\left(\phi_{k}(z)\right) & \approx\left[g(1)+\frac{g^{\prime \prime}(1)}{2}(1-z)^{2}\right]-\left[g(1)+\frac{g^{\prime \prime}(1)}{2}\left(1-\phi_{k}(z)\right)^{2}\right]= \\
& =\frac{G}{2}\left[2-z-\phi_{k}(z)\right]\left(z-\phi_{k}(z)\right) \geq G \cdot \delta \cdot\left(z-\phi_{k}(z)\right)
\end{aligned}
$$

where the first $\approx$ refers to a second-order approximation. (21) then gives

$$
z-\phi_{k}(z) \approx \leq \frac{g(z)-g\left(\phi_{k}(z)\right)}{G \cdot \delta}=\frac{\left(\mu_{k+1}-\mu_{k}\right) \cdot \phi_{k}(z)}{\nu_{k+1} \cdot G \cdot \delta} \leq \frac{1}{G \cdot \delta} \cdot \frac{\left(\mu_{k+1}-\mu_{k}\right)}{\nu_{k+1}}
$$

If we had $x_{k}^{n} \underset{n \rightarrow \infty}{\rightarrow} 0$, it's easy to see that if $\delta>0$ was chosen small enough, then it holds 
for each large enough $n$, there is $k_{n}$ with $x_{k_{n}}^{n} \in(\delta, 1-\delta)$. But if $\left(\frac{\left(\mu_{k+1}-\mu_{k}\right)}{\nu_{k+1}}\right)_{k=1}^{\infty}$ is summable, if $n$ is large enough for this to hold, and if $\sum_{j>K} \frac{1}{G \cdot \delta} \cdot \frac{\left(\mu_{k+1}-\mu_{k}\right)}{\nu_{k+1}}<\frac{\delta}{2}$

$$
\begin{aligned}
x_{K}^{n} & =\left(\phi_{K}\left(\cdots\left(\phi_{k_{n}}\left(x_{k_{n}}^{n}\right)\right) \cdots\right)\right)=x_{k_{n}}^{n}-\left[x_{k_{n}}^{n}-\phi_{k_{n}}\left(x_{k_{n}}^{n}\right)\right]-\left[\phi_{k_{n}}\left(x_{k_{n}}^{n}\right)-\phi_{k_{n}-1}\left(\phi_{k_{n}}\left(x_{k_{n}}^{n}\right)\right]-\cdots\right. \\
& -\left[\phi_{K+1}\left(\cdots\left(\phi_{k_{n}}\left(x_{k}^{n}\right)\right) \cdots\right)-\phi_{K}\left(\phi_{K+1}\left(\cdots\left(\phi_{k_{n}}\left(x_{k_{n}}^{n}\right)\right) \cdots\right)\right] \geq \delta-\frac{\delta}{2}=\frac{\delta}{2}\right.
\end{aligned}
$$

hence, $x_{K} \geq \frac{\delta}{2}>0$.

We observe that the analysis above shows that $x_{k}^{n}$ which is purchased under $\left(p^{n}, \alpha^{n}\right)$ by type $\left(\mu^{k}, \nu^{k}\right)$ satisfies $x_{k}^{n}:=\phi_{k}\left(\phi_{k+1}\left(\cdots\left(\phi_{n}(1)\right) \cdots\right)\right)$; hence, limit $x_{k}:=\lim _{n \rightarrow \infty} x_{k}^{n}$ exists since $\left(x_{k}^{n}\right)_{n \geq k}$ is monotonically decreasing and $x_{k}>0$ for each $k$ by the above argument. Let $(p, \alpha)$ be the candidate equilibrium, in $\left(\mu^{k}, \nu^{k}\right)$ purchases coverage $x_{k}$ and price $\mu_{k} x_{k}$ - as described in Section 4.4. The proof of the required convergence - ie., $p^{n} \rightarrow p$ uniformly on compact subsets of $[0,1)$, and $\alpha^{n} \rightarrow \alpha$ - follows along lines similar to the continuous case, and hence it is presented in Online Appendix K.

To show Proposition 3, we see that if $z-\phi_{k}(z) \geq 1-\delta$, we have $g(z)-g\left(\phi_{k}(z)\right) \approx \leq$ $G\left(z-\phi_{k}(z)\right)$, so $z-\phi_{k}(z) \approx \geq \frac{1}{G} \cdot \frac{\left(\mu_{k+1}-\mu_{k}\right)}{\nu_{k+1}}$. If $\left(\frac{\mu_{k+1}-\mu_{k}}{\nu_{k+1}}\right)_{k=1}^{\infty}$ is not summable, the argument above shows for each $k$, if $n$ is large enough, $x_{k}^{n} \leq 1-\delta$ so as to be outside of this domain $(1-\delta, 1)$ in which the approximation is valid; so $\left(x_{k}\right)_{k=1}^{\infty}$ all lie in $[0,1-\delta]$, contradicting Theorem 2 (full coverage in equilibrium support); the other conclusions of Proposition 3 can follow using similar approximations. 


\section{References}

George A. Akerlof. The market for "lemons": Quality uncertainty and the market mechanism. Quarterly Journal of Economics, 84(3):488-500, 1970.

Eduardo M Azevedo and Daniel Gottlieb. An example of non-existence of riley equilibrium in markets with adverse selection. 2016.

Eduardo M. Azevedo and Daniel Gottlieb. Perfect competition in markets with adverse selection. Econometrica, 85(1):67-105, 2017.

Jason Brown, Mark Duggan, Ilyana Kuziemko, and William Woolston. How does risk selection respond to risk adjustment? new evidence from the medicare advantage program. The American Economic Review, 104(10):3335-3364, 2014.

Keith J Crocker and Arthur Snow. Multidimensional screening in insurance markets with adverse selection. Journal of Risk and Insurance, 78(2):287-307, 2011.

David De Meza and David C Webb. Advantageous selection in insurance markets. RAND Journal of Economics, pages 249-262, 2001.

Gerard Debreu. Economies with a finite set of equilibria. Econometrica, pages 387-392, 1970.

Pradeep Dubey and John Geanakoplos. Competitive pooling: Rothschild-stiglitz reconsidered. Quarterly Journal of economics, pages 1529-1570, 2002.

Liran Einav, Amy Finkelstein, and Mark R. Cullen. Estimating welfare in insurance markets using variation in prices. Quarterly Journal of Economics, 125(3):877-921, 2010.

Irina Gemmo, Christian Kubitza, and Casey G Rothschild. The existence of the miyazakiwilson-spence equilibrium with continuous type distributions. Technical report, ICIR Working Paper Series, 2018.

Ben Handel, Igal Hendel, and Michael D Whinston. Equilibria in health exchanges: Adverse selection versus reclassification risk. Econometrica, 83(4):1261-1313, 2015.

Nathaniel Hendren. Private information and insurance rejections. Econometrica, 81(5): 1713-1762, 2013.

John L Kelley. General topology. Courier Dover Publications, 2017.

Yehuda Levy and Andre Veiga. Frictions and equilibria in insurance markets. Working Paper, 2017. 
George J Mailath, Masahiro Okuno-Fujiwara, and Andrew Postlewaite. Belief-based refinements in signalling games. Journal of Economic Theory, 60(2):241-276, 1993.

James A. Mirrlees. An exploration in the theory of optimum income taxation. Review of Economic Studies, 38(2):175-208, 1971.

Hajime Miyazaki. The rat race and internal labor markets. The Bell Journal of Economics, 8(2):394-418, 1977.

John G. Riley. Informational equilibrium. Econometrica, 47(2):331-359, 1979.

Michael Rothschild and Joseph E. Stiglitz. Equilibrium in competitive insurance markets: An essay on the economics of imperfect information. Quarterly Journal of Economics, 90(4):629-649, 1976.

Walter Rudin. Real and complex analysis. Tata McGraw-Hill Education, 1987.

Michael Smart. Competitive insurance markets with two unobservables. International Economic Review, 41(1):153-170, 2000.

Arthur Snow. On the possibility of profitable self-selection contracts in competitive insurance markets. Journal of Risk and Insurance, 76(2):249-259, 2009.

A. Michael Spence. Job market signaling. Quarterly Journal of Economics, 87(3):355, 1973.

Michael Spence. Product differentiation and performance in insurance markets. Journal of Public Economics, 10(3):427-447, 1978.

Andre Veiga and E Glen Weyl. Product design in selection markets. Quarterly Journal of Economics, 2016.

Bertrand Villeneuve. Concurrence et antisélection multidimensionnelle en assurance. Annales d'Economie et de Statistique, pages 119-142, 2003.

Achim Wambach. Introducing heterogeneity in the rothschild-stiglitz model. Journal of Risk and Insurance, 67(4):579-591, 2000.

Charles Wilson. A model of insurance markets with incomplete information. Journal of Economic Theory, 16(2):167-207, 1977. 


\section{Appendix for Online Publication}

\section{G Existence in General Insurance}

We now prove Theorem 9: equilibrium existence in insurance markets exists under conditions more general than those stated in Section 4.

Fix a sequence of compact subsets $\Theta_{1} \subseteq \Theta_{2} \subseteq \cdots \Theta$ with $\Theta=\cup \Theta_{n}$. Let $M^{n}$ be the essential supremum of $\mu$ w.r.t. $P\left(\cdot \mid \Theta^{n}\right)$. Then $M^{1} \leq M^{2} \leq M^{3} \leq \cdots$ with $M^{n} \rightarrow \infty$, $M^{n} \in \operatorname{supp}\left(P_{\mu}\right)$ for each $n \in \mathbb{N}$. Let $P^{n}=P\left(\cdot \mid \Theta_{n}\right)$, and $\bar{X}=[0,1]$. By AG, $\mathcal{E}^{n}=\left[\Theta^{n}, \bar{X}, P^{n}\right]$ has an equilibrium.

Fix one such equilibrium $\left(\alpha^{n}, p^{n}\right)$ for each economy. Proposition 8 implies that in each of these equilibria, there is a strictly increasing function $\sigma^{n}: \operatorname{supp}\left(P_{\mu}^{n}\right) \rightarrow[0,1]$ such that type with riskiness $\mu \in \operatorname{supp}\left(P_{\mu}^{n}\right)$ purchases coverage $\sigma^{n}(\mu) \alpha^{n}$-a.s., with $\sigma^{n}\left(M^{n}\right)=1$.

We will require the following two results.

Lemma 14. For each $\mu \in \operatorname{supp}\left(P_{\mu}\right), \lim \sup _{n \rightarrow \infty} \sigma^{n}(\mu)<1$.

The intuition is simple: Fixing $\mu_{1}<\mu_{2}$, then $\sigma^{n}\left(\mu_{1}\right) \leq \sigma^{n}\left(\mu_{2}\right)$ for all $n$, and if $\sigma^{n}\left(\mu_{2}\right)$ is close to 1 , then $\sigma^{n}\left(\mu_{1}\right)$ should not be more (approximately) than the coverage $z$ that makes type $\mu_{2}$ indifferent between contract $\left(x=1, p=\mu_{2}\right)$ and $\left(z, \mu_{1} \cdot z\right)$.

Proof. For each $\theta_{2}, \theta_{1}$ with $\mu_{2}=\mu\left(\theta_{2}\right)>\mu_{1}=\mu\left(\theta_{1}\right)$ and each $u \in[0,1]$, let $\phi\left(\mu_{1}, \mu_{2}, u\right)$ denote the unique $v<u$ s.t. type $\theta_{2}$ is indifferent between contracts $\left(\mu_{2} \cdot u, u\right)$ and $\left(\mu_{1} \cdot v, v\right)$; such unique $v$ exists as $w_{\theta_{2}}(x)>\mu_{2}>\mu_{1}$ for all $x \in(0,1), \phi$ is continuous on $\mathbb{R}_{++} \times \mathbb{R}_{++} \times[0,1]$, and $\phi\left(\mu_{1}, \mu_{2}, u\right)<u$ for $u \in(0,1]$.

Suppose $\mu_{1} \in \operatorname{supp}\left(P_{\mu}\right)$ with $\lim _{n \rightarrow \infty} \sigma^{k_{n}}\left(\mu_{1}\right)=1$ for some indices $\left(k_{n}\right)$. Fix some $\mu_{2}>\mu_{1}$ with $\mu_{2} \in \operatorname{supp}\left(P_{\mu}\right)$, and hence w.l.o.g., $\mu_{2} \in \operatorname{supp}\left(P_{\mu}^{k_{n}}\right)$ for all $n \in \mathbb{N}$. For each $k_{n}$, $\sigma^{k_{n}}\left(\mu_{1}\right) \leq \phi\left(\mu_{1}, \mu_{2}, \sigma^{k_{n}}\left(\mu_{2}\right)\right)$, as otherwise type $\theta$ with $\mu(\theta)=\mu_{1}$ would instead choose coverage $\sigma^{k_{n}}\left(\mu_{2}\right)$. Then $\lim _{n \rightarrow \infty} \sigma^{k_{n}}\left(\mu_{2}\right)=1$ as each $\sigma^{k_{n}}$ is monotonically increasing. Hence,

$$
1=\lim _{n \rightarrow \infty} \sigma^{k_{n}}\left(\mu_{1}\right) \leq \lim _{n \rightarrow \infty} \phi\left(\mu_{1}, \mu_{2}, \sigma^{k_{n}}\left(\mu_{2}\right)\right)=\phi\left(\mu_{1}, \mu_{2}, \lim _{n \rightarrow \infty} \sigma^{k_{n}}\left(\mu_{2}\right)=1\right)=u\left(\mu_{1}, \mu_{2}, 1\right)<1
$$

a contradiction.

Notice that Lemma 14 does not rely on the condition given in Equation (11). Lemma 15 however, crucially, does:

Lemma 15. For each $0<m<1$, there is $M>0$ such that if $\mu>M$ and $n \in \mathbb{N}$ is such that $\mu \in \operatorname{supp}\left(P_{\mu}^{n}\right)$, then $\sigma^{n}(\mu)>m$. 
The idea is to make observe types purchasing a sequence $\mu_{1}<\mu_{2}<\cdots$, and bound the differences between the coverages chosen by two adjacent types in this sequence. (It is tempting to think we are making a reduction to the discrete case, but since the utility has a more general form that in Section 4.4, the arguments have to be slightly less direct.)

Proof. Fix types $\theta^{\prime \prime}, \theta^{\prime}$ and denote $\mu^{\prime \prime}:=\mu\left(\theta^{\prime \prime}\right)>\mu^{\prime}:=\mu\left(\theta^{\prime}\right), x^{\prime \prime}:=\sigma\left(\mu^{\prime \prime}\right)>x^{\prime}:=\sigma\left(\mu^{\prime}\right) \geq$ $1-\delta$, and $\nu^{\prime \prime}:=\underline{\nu}_{\delta}\left(\theta^{\prime \prime}\right), \nu^{\prime}:=\underline{\nu}_{\delta}\left(\theta^{\prime}\right)$. Denote

$$
\begin{gathered}
\Delta \mu=\mu^{\prime \prime}-\mu^{\prime} \quad \Delta x=x^{\prime \prime}-x^{\prime} \\
\Delta p=p\left(x^{\prime \prime}\right)-p\left(x^{\prime}\right)=\mu^{\prime \prime} \cdot x^{\prime \prime}-\mu^{\prime} \cdot x^{\prime}=\mu^{\prime} \cdot \Delta x+x^{\prime \prime} \cdot \Delta \mu
\end{gathered}
$$

It follows from Lemma 9 of Section D.1 that

$$
\Delta p \geq \Delta x \cdot\left[\mu^{\prime}+\nu^{\prime} \cdot\left(1-\frac{x^{\prime}+x^{\prime \prime}}{2}\right)\right]
$$

Combining these,

$$
x^{\prime \prime} \cdot \Delta \mu \geq \Delta x \cdot \nu^{\prime} \cdot\left(1-\frac{x^{\prime}+x^{\prime \prime}}{2}\right) \rightarrow \Delta x \leq \frac{\Delta \mu}{\nu^{\prime}}\left(1-\frac{x^{\prime}+x^{\prime \prime}}{2}\right)^{-1}
$$

Hence,

$$
\Delta x \leq \frac{\Delta \mu}{\nu^{\prime}} \cdot \frac{2}{2-x^{\prime}-x^{\prime \prime}}<\frac{\Delta \mu}{\nu^{\prime}} \cdot \frac{1}{1-x^{\prime \prime}}
$$

Also, since $2-x^{\prime}-x^{\prime \prime} \geq x^{\prime \prime}-x^{\prime}=\Delta x$,

$$
\Delta x \leq \frac{\Delta \mu}{\nu^{\prime}} \cdot \frac{2}{\Delta x} \rightarrow \Delta x \leq \sqrt{2 \cdot \frac{\Delta \mu}{\nu^{\prime}}}
$$

Now, by (11), there exists a sequence of types $\left(\theta_{j}\right)$ such that, denoting $\mu_{j}=\mu\left(\theta_{j}\right)$, we have $\mu_{1}<\mu_{2}<\cdots$, and such that denoting $\nu_{n}=\underline{\nu}_{\delta}\left(\theta_{n}\right)$,

$$
\sum_{n=1}^{\infty} \frac{\mu_{n+1}-\mu_{n}}{\nu_{n}}<\infty
$$

Fix $m, \eta>0$ with $m<1-4 \eta<1$, and choose $K$ such that

$$
\sum_{n=K}^{\infty} \frac{\mu_{n+1}-\mu_{n}}{\nu_{n}}<\eta^{2}
$$

Each term is hence also less than $\eta^{2}$. Suppose by way of contradiction, $\sigma\left(\theta_{K}\right) \leq m$. Then 
for each $n \geq K$, if $x_{n+1}>1-\eta$ we can say

$$
x_{n+1}-x_{n} \leq \sqrt{2 \cdot \eta^{2}}<2 \eta
$$

while if $x_{n+1} \leq 1-\eta$, we can say

$$
x_{n+1}-x_{n} \leq \frac{1}{\eta} \cdot \frac{\mu_{n+1}-\mu_{n}}{\nu_{n}}
$$

From the first of these, we see that there is $N>K$ such that $1-3 \eta<x_{N}<1-\eta$. Then,

$$
x_{N}-x_{K} \leq \frac{1}{\eta} \sum_{n=K}^{N} \frac{\mu_{n+1}-\mu_{n}}{\nu_{n}} \leq \frac{1}{\eta} \cdot \eta^{2} \rightarrow x_{K} \geq x_{N}-\eta>(1-3 \eta)-\eta>m
$$

a contradiction. Hence, we may take $M=\mu_{K}$.

As a result, the conditions of Proposition 6 hold: As remarked there, Assumption 1 - in particular, the quasi-linearity of utility in prices - implies that for every two alternatives $x, y \in X$, price $p \geq 0$, and type $\theta \in \Theta$, there is price $q$ high enough s.t. $u(\theta, x, p)>u(\theta, y, q)$. As for the required properties of the equilibria $\left(p_{n}, \alpha_{n}\right)_{n=1}^{\infty}$ :

1. First we show that if $[0, m] \subseteq[0,1)$, then $\left(p_{n}\right)$ is point-wise (in fact, uniformly) bounded and equicontinuous on $[0, m]$ : Choose some $\theta_{0} \in \Theta$ such that, denoting $\mu_{0}=\mu\left(\theta_{0}\right), \sigma^{n}\left(\mu_{0}\right)>m$ whenever $\mu_{0} \in \operatorname{supp}\left(P_{\mu}^{n}\right)$. Such $\theta_{0}$ exists by Lemma 15 . Then for all such $n$ and all $x \leq m, p_{n}(x) \leq p_{n}(m) \leq \mu_{0} \cdot m$, so we have the boundedness in $[0, m]$. Denote $w \equiv w_{\theta_{0}}$. Then for $\alpha$-a.e. type $\theta$ that choose coverage in $[0, m], \sigma(\mu(\theta)) \leq \sigma\left(\mu_{0}\right)=\sigma\left(\mu\left(\theta_{0}\right)\right)$ so by Assumption 10, $w_{\theta} \leq w_{\theta_{0}}$, and each type has Lipschitz utility (with the same Lipschitz constant as $w_{\theta_{0}}$.)

2. Next we verify that for each $M \in \mathbb{R}$, there is a compact $K \subseteq[0,1)$, s.t. for large enough $n, \inf _{x \notin K} p^{n}(x) \geq M$. Fix $M>0$ and some $\mu \in \operatorname{supp}\left(P_{\mu}\right)$ with $\mu>2 M$, let $N \in \mathbb{N}$ and $\frac{1}{2}<t<1$ be such that $\sigma^{n}(\mu)<t$ for all $n>N$; such $N, t$ are guaranteed by Lemma 14. By the monotonicity of each $\sigma^{n}$ and $p^{n}$, for $n>N$ and $x \notin K:=[0, t]$,

$$
p^{n}(x)>p^{n}(t) \geq p^{n}\left(\sigma^{n}(\mu)\right)=\sigma^{n}(\mu) \cdot \mu \geq t \cdot 2 M>M
$$

3. Lemma 1 shows that Condition (1) (required by Proposition 6) holds.

Hence, an equilibrium $(p, \alpha)$ of the economy $[X, \Theta, P]$ exists. 


\section{H Generalized Equilibrium Properties}

This section shows that several properties of equilibrium derived by AG also hold in settings where costs are unbounded. (Note that Lemma 16 is used, implicitly or explicitly, at several points in the various proofs.) Proposition 14 is used implicitly throughout, in particular the fact that in equilibrium, a.e. agent is selecting an optimal contract.

\section{H.1 Continuity}

The continuity (and in fact Lipschitz-ness) of prices was proven in AG (for the bounded environments they consider). Here, we prove the continuity of prices in generic unbounded settings. ${ }^{60}$

Lemma 16. If $(p, \alpha)$ is an AG-equilibrium, then $p$ is continuous.

Proof. Suppose $x_{n} \rightarrow x$ in $X$, and let $\left(p^{n}, \alpha^{n}\right)_{n=1}^{\infty}$ be the approximating sequence of weak equilibria with alternatives $\left(\bar{X}_{n}\right)_{n=1}^{\infty}$. By passing to a subsequence of $\left(p^{n}, \alpha^{n}\right)$, we may assume that for each $n$, there is $y_{n} \in \bar{X}_{n}$ such that

$$
\left|y_{n}-x_{n}\right|<\frac{1}{n} \text { and }\left|p^{n}\left(y_{n}\right)-p\left(x_{n}\right)\right|<\frac{1}{n}
$$

Hence, $y_{n} \rightarrow x$. Therefore, since $\left(p^{n}, \alpha^{n}\right)_{n=1}^{\infty}$ witnesses that $(p, \alpha)$ is an equilibrium, $p^{n}\left(y_{n}\right) \rightarrow p(x)$. By the second inequality, $p\left(x_{n}\right) \rightarrow p(x)$, as required.

\section{H.2 Equilibrium is Weak Equilibrium}

AG shows under their weaker assumptions that equilibria are, in particular, weak-equilibrium. This will also be true in our case although, to prove it, we need the following auxiliary result.

The following lemma, stated in greater generality than needed, may be of independent interest.

Lemma 17. Let $X$ be a locally compact separable metric space, $\left(X_{n}\right)$ a sequence of finite subsets, $p: X \rightarrow \mathbb{R}$ continuous and for each $n \in \mathbb{N}, p_{n}: X_{n} \rightarrow \mathbb{R}$, s.t. if $\left(x_{n}\right)$ is a sequence in $X$ with $x_{n} \rightarrow x \in X$ s.t. $x_{n} \in X_{n}$ for each $n \in \mathbb{N}$, then $p_{n}\left(x_{n}\right) \rightarrow p(x)$.

Then there are extensions of the $p_{n}$ to continuous functions $\tilde{p}_{n}: X \rightarrow \mathbb{R}_{+}$s.t. $\tilde{p}_{n} \rightarrow p$ uniformly on compact sets. In particular, if $X$ is compact, then $\forall \varepsilon>0$, there is $N \in \mathbb{N}$, s.t. $\forall n>N$ and $\forall x \in X_{n},\left|p_{n}(x)-p(x)\right|<\varepsilon$.

\footnotetext{
${ }^{60}$ The continuity of prices in the particular case of the utility functions of insurance markets discussed in this paper, as introduced in Section 6.2, follow from Lemma 11. 16 holds in a much more general setup.
} 
The latter conclusion, for the case of compact $X$, follows from the first part. We note, however, that the latter conclusion actually already follows from the first step in the proof.

Proof. Let $\left(K_{j}\right)_{j=1}^{\infty}$ be an increasing sequence of compact sets with $X=\cup_{j} K_{j}$ and $K_{j} \subseteq$ $K_{j+1}^{\circ}$; such exists as $X$ is locally compact and separable metric. Fix $J \in \mathbb{N}$ : We contend that $\forall \varepsilon>0$, there is $N \in \mathbb{N}$, s.t. $\forall n>N$ and $\forall x \in X_{n} \cap K_{J},\left|p_{n}(x)-p(x)\right|<\varepsilon$. Indeed, if not, there is $\varepsilon>0$, a sequence $n_{1}<n_{2}<\cdots$ of indices, a sequence $\left(x_{j}\right)$ with $x_{j} \in$ $X_{n_{j}} \cap K_{J},\left|p_{n_{j}}\left(x_{j}\right)-p\left(x_{j}\right)\right| \geq \varepsilon$, and such that $\left(x_{j}\right)$ converges; denote the limit $x \in K_{J}$. Hence, $p_{n_{j}}\left(x_{j}\right) \rightarrow p(x)$ by assumption. Since $p$ is continuous by Lemma 16, $p\left(x_{j}\right) \rightarrow p(x)$. Together, these give a contradiction.

Hence, define $q_{n}: X_{n} \rightarrow \mathbb{R}$ by $q_{n}=p_{n}-p$. Denote $Y_{n}=X_{n} \cap K_{n}, \varepsilon_{n}=\max _{x \in Y_{n}}\left|q_{n}\right|$. By the last paragraph, $\varepsilon_{n} \rightarrow 0$. The Tietze extension theorem implies, for each $n \in \mathbb{N}$, the existence of a continuous extension $\tilde{q}_{n}$ of $q_{n}$ to $X$ satisfying $\varepsilon_{n}=\max _{K_{n}}\left|\tilde{q}_{n}\right|$. (Formally, first extend the restriction of $q_{n}$ to $Y_{n}$ to a function $\tilde{q}_{n}$ on $K_{n}$ satisfying $\varepsilon_{n}=\max _{K_{n}}\left|\tilde{q}_{n}\right|$ via Tietze's theorem, and then extend it to a function on $X$ agreeing with $q_{n}$ on $X_{n}$ in an arbitrary continuous way, again via Tietze's theorem.) Defining $\tilde{p}_{n}=\tilde{q}_{n}+p$ for each $n \in \mathbb{N}$ give the required extensions, since for any compact subset $K \subseteq X$, there is $J$ s.t. for all $j>J, K \subseteq K_{j}$.

Now, the proof of Proposition 14 follows along lines similar to the corresponding Proposition in AG, with some care required since our setup allows for unbounded cost.

\section{Proposition 14. An equilibrium is also a weak equilibrium.}

Proof. Take a sequence $\left(X_{n}, p_{n}, \alpha_{n}\right)$ of weak equilibria on finite subsets $X_{n} \subseteq X$ which witnesses that $(p, \alpha)$ is an equilibrium. Let $\left(\tilde{p}_{n}\right)$ correspond to $\left(p_{n}\right),\left(X_{n}\right)$, and $p$ as in Lemma 17. For any continuous function $f: X \rightarrow \mathbb{R}$ with compact support, since the $\tilde{p}_{n}$ are uniformly bounded on compact sets ( $p$ is continuous and $\tilde{p}_{n} \rightarrow p$ uniformly on compact sets), and since $\tilde{p}^{n}(x)=p^{n}(x)=E_{\alpha^{n}}[c \mid x]$ for all $x \in \operatorname{supp}\left(\alpha^{n}\right)$,

$$
\begin{aligned}
\int_{\Theta \times X} f \cdot p \cdot d \alpha & =\lim _{n \rightarrow \infty} \int_{\Theta \times X} f \cdot p \cdot d \alpha^{n}=\lim _{n \rightarrow \infty} \int_{\Theta \times X} f \cdot \tilde{p}^{n} \cdot d \alpha^{n} \\
& =\lim _{n \rightarrow \infty} \int_{\Theta \times X} f(x) \cdot c(x, \theta) d \alpha^{n}(x, \theta)
\end{aligned}
$$

Denoting by $K \subseteq X$ the compact support of $f$. Recall that by assumption,

$$
\int_{\Theta} \max _{x \in K} c(x, \theta) d P(\theta)<\infty
$$

so for each $\varepsilon>0$, there is compactly support $g_{\varepsilon}: \Omega \rightarrow[0,1]$ s.t.

$$
\int_{\Theta}\left(1-g_{\varepsilon}(\theta)\right) \max _{x \in K} c(x, \theta) d P(\theta)<\varepsilon .
$$


Clearly,

$$
\begin{aligned}
\int_{\Theta \times X} f(x) \cdot c(x, \theta) d \alpha^{n}(x, \theta) & =\int_{\Theta \times X} f(x) g_{\varepsilon}(\theta) \cdot c(x, \theta) d \alpha^{n}(x, \theta) \\
& +\int_{\Theta \times X} f(x)\left(1-g_{\varepsilon}(\theta)\right) \cdot c(x, \theta) d \alpha^{n}(x, \theta) \\
\int_{\Theta \times X} f(x) \cdot c(x, \theta) d \alpha(x, \theta) & =\int_{\Theta \times X} f(x) g_{\varepsilon}(\theta) \cdot c(x, \theta) d \alpha(x, \theta) \\
& +\int_{\Theta \times X} f(x)\left(1-g_{\varepsilon}(\theta)\right) \cdot c(x, \theta) d \alpha(x, \theta)
\end{aligned}
$$

Now,

$$
\lim _{n \rightarrow \infty} \int_{\Theta \times X} f(x) g_{\varepsilon}(\theta) \cdot c(x, \theta) d \alpha^{n}(x, \theta)=\int_{\Theta \times X} f(x) g_{\varepsilon}(\theta) \cdot c(x, \theta) d \alpha(x, \theta)
$$

and the errors terms are at most $\varepsilon \cdot \sup |f|$, and $\varepsilon>0$ was arbitrary. Hence,

$$
\int_{\Theta \times X} f(x) \cdot p(x) d \alpha(x, \theta)=\int_{\Theta \times X} f(x) \cdot c(x, \theta) d \alpha(x, \theta)
$$

and this was for any $f: X \rightarrow \mathbb{R}$ compactly supported. Hence, $p(x)=E_{\alpha}[c(x, \theta) \mid x] \alpha$-a.s.

Now, let $\phi: \mathbb{R} \rightarrow \mathbb{R}$ be a strictly monotonically increasing continuous funding with bounded range, e.g., $\phi(x)=\arctan (x)$ or $\phi(x)=\frac{x}{1+|x|}$. Since $\alpha_{n}$ is a weak equilibrium, it holds

$$
u\left(\theta, \tilde{p}_{n}(x), x\right)=\sup _{x^{\prime} \in X_{n}} u\left(\theta, \tilde{p_{n}}\left(x^{\prime}\right), x^{\prime}\right), \text { for } \alpha_{n}-\text { a.e. }(\theta, x) \in \Theta \times X_{n}
$$

Hence, it is also true that, denoting $v=\phi \circ u$

$$
v\left(\theta, \tilde{p}_{n}(x), x\right)=\sup _{x^{\prime} \in X_{n}} v\left(\theta, \tilde{p_{n}}\left(x^{\prime}\right), x^{\prime}\right), \text { for } \alpha_{n}-\text { a.e. }(\theta, x) \in \Theta \times X_{n}
$$

Let $\alpha^{\prime}$ be a 'deviation to $\alpha^{\prime}$ - i.e., a measure on $\Theta \times X$ whose projection to $\Theta$ is $P$, and letting $\left(\alpha_{n}^{\prime}\right)$ be a sequence of measures on $(\Theta \cup X) \times X$, with $\alpha_{n}^{\prime}$ supported on $\left(\Theta \cup X_{n}\right) \times X_{n}$ and $\alpha_{n}^{\prime} \rightarrow \alpha$ weakly, we have since $\left(p_{n}, \alpha_{n}\right)$ is a weak equilibrium,

$$
\int_{\Theta \times X} v\left(\theta, \tilde{p}_{n}(x), x\right) d \alpha_{n} \geq \int_{\Theta \times X} v\left(\theta, \tilde{p}_{n}(x), x\right) d \alpha_{n}^{\prime}
$$

$\alpha_{n} \rightarrow \alpha, \alpha_{n}^{\prime} \rightarrow \alpha^{\prime}$, so the families $\left(\alpha_{n}\right)$ and $\left(\alpha_{n}^{\prime}\right)$ are tight, and $v$ is bounded. Hence, for each $\varepsilon>0$, there is $\zeta_{\varepsilon}: \Theta \times X \rightarrow[0,1]$ continuous and compactly supported, such that

$$
\int_{\Omega \times X}\left(1-\zeta_{\varepsilon}(x, \theta)\right) \cdot\left|v\left(\theta, \tilde{p}_{n}(x), x\right)\right| d \beta<\varepsilon, \text { for any } \beta=\alpha_{n}, \alpha, \alpha_{n}^{\prime}, \alpha^{\prime}, n \in \mathbb{N}
$$


Since $\tilde{p}_{n} \rightarrow p$ uniformly on the support of $\zeta_{\varepsilon}$,

$$
\int_{\Theta \times X} \zeta_{\varepsilon}(x, \theta) v\left(\theta, \tilde{p}_{n}(x), x\right) d \alpha_{n} \rightarrow \int_{\Theta \times X} \zeta_{\varepsilon}(x, \theta) v(\theta, p(x), x) d \alpha
$$

and

$$
\int_{\Theta \times X} \zeta_{\varepsilon}(x, \theta) v\left(\theta, \tilde{p}_{n}(x), x\right) d \alpha_{n}^{\prime} \rightarrow \int_{\Theta \times X} \zeta_{\varepsilon}(x, \theta) v(\theta, p(x), x) d \alpha^{\prime}
$$

Since this was for any compactly supported $\zeta_{\varepsilon}$, it follows that

$$
\int_{\Theta \times X} v(\theta, p(x), x) d \alpha \geq \int_{\Theta \times X} v(\theta, p(x), x) d \alpha^{\prime}
$$

Since this was for any measure $\alpha^{\prime}$ on $\Theta \times X$ whose projection to $\Theta$ is $P$,

$$
v(\theta, p(x), x)=\sup _{x^{\prime} \in X} v\left(\theta, p\left(x^{\prime}\right), x^{\prime}\right), \text { for } \alpha-\text { a.e. }(\theta, x) \in \Theta \times X
$$

and therefore

$$
u(\theta, p(x), x)=\sup _{x^{\prime} \in X} u\left(\theta, p\left(x^{\prime}\right), x^{\prime}\right), \text { for } \alpha-\text { a.e. }(\theta, x) \in \Theta \times X
$$

\section{Discrete types: Direct Construction}

In this Section we provide a "direct" construction of the AG equilibrium for a simple insurance economy with discrete types, without using Proposition 1.

\section{I.1 Equilibrium in bounded economies}

We now show that, for a truncated economy $\mathcal{E}^{n}$, the allocation described in Proposition 4 is indeed an AG equilibrium.

Proof. Since utilities are quasi-linear and $p>0$ on $(0,1),{ }^{61}$ it is enough to approximate $(p, \alpha)$, in the same manner described in Section 1, but on $X^{\prime}=\left(x_{0}, 1\right)$ instead of $[0,1)$ (as $p \equiv 0$ in $\left.\left(0, x_{0}\right)\right)$ and with $\eta_{n}$ not necessarily strictly positive on the behavioral types $\bar{X}^{n}$; afterwards the weight of the behavioral types could be increased slightly to be strictly positive in such a way that the price goes down by the same amount for each alternative in $\bar{X}^{n}$.

\footnotetext{
${ }^{61} p(0)=0$, but $\forall n, \bar{X}^{n} \subseteq(0,1)$ in our construction to follow, so $p$ is positive on $\bar{X}^{n}$.
} 
We will also index the sequence of economies by $n$. For each $n$, let $\bar{X}^{n}$ be the set

$$
\bar{X}^{n}=\left\{x_{i j} \mid i=1, \ldots, n, j=1, \ldots, n\right\} \cup\left\{x_{1}, \ldots, x_{n}\right\} .
$$

The contracts $x_{1}, \ldots, x_{n}$ are obtained as in Section 4.4, the contracts purchased by the first $n$ types. That is, economy $n$ has only the first $n$ contracts $x_{1}, \ldots, x_{n}$. Moreover, to each contract $i$ are associated $n$ behavioral types $x_{i 1}, \ldots, x_{i n}$ are distributed (e.g., evenly) strictly between $x_{i-1}$ and $x_{i}$. (Recall that $x_{0}$ is the right-most point s.t. $p\left(x_{0}\right)=0$, i.e., where type 1 is indifferent between $\left(0, x_{0}\right)$ and $\left(p_{1}, x_{1}\right)$.) The mass of agents at each $x_{i j}$ (which denote $\eta_{n}$ ) is defined below.

As in AG, the behavioral agents in $\bar{X}^{n}$ have riskiness $\mu=0$, i.e., zero cost. We set prices $p_{n} \equiv p$ for contracts on $\bar{X}^{n}$. Moreover, we set the distribution of the weak equilibrium $\left(\alpha_{n}\right)$ such that

$$
\begin{aligned}
& \alpha_{n}\left(\left\{\mu_{i}, x_{i}\right\}\right)=P_{i}\left[1-\frac{1}{n}\right], \forall i=1, \ldots, n \\
& \alpha_{n}\left(\left\{\mu_{i+1}, x_{i j}\right\}\right)=P_{i+1} \frac{1}{n^{2}}, \forall i, j=1, \ldots, n
\end{aligned}
$$

That is, of the original mass $P_{i}$ of "regular" types $\mu_{i}$, all but a $\frac{1}{n}$-fraction choose $x_{i}$, while the rest evenly spread themselves between the contracts $x_{i, 1}, \ldots, x_{i, n}$, such that the mass of type $\mu_{i}$ in each of these contracts is a share $\frac{1}{n^{2}}$ of the total mass $P_{i}$. Recall that $x_{i-1}<$ $x_{i 1}<\ldots<x_{i, n}<x_{i}$ and moreover $p(x)$ is defined so that types $\mu_{i}$ are indifferent between all these contracts.

We also construct the distribution $\alpha_{n}$ such that, all types $k>n$ (each with mass $P_{k}$ ) purchase the highest coverage available $\left(x_{n}\right)$ :

$$
\alpha_{n}\left(\left\{\mu_{k}, x_{n}\right\}\right)=P_{k}, \quad \forall k>n
$$

Since $\mu_{n}, \nu_{n}$ increasing, this maximizes their utility when contracts $x_{k}$ for $k>n$ are not available. This construction of $\alpha_{n}$ is illustrated by Figure 6 .

We then define $\alpha_{n}\left(\left\{\left(x_{i j}, x_{i j}\right)\right\}\right)=\eta_{n}\left(\left\{x_{i j}\right\}\right)$ for all $i, j$ to be the mass of behavioral types who purchase contract $x_{i j}$ (which, recall, will also be purchased by some mass of types $\left.\mu_{i}\right)$. We define $\eta_{n}\left(x_{i j}\right)$ to satisfy

$$
g_{i}\left(x_{i j}\right)=x_{i j} \mu_{i} \frac{P_{i} \cdot \frac{1}{n^{2}}}{P_{i} \cdot \frac{1}{n^{2}}+\eta_{n}\left(x_{i j}\right)} \leq 1 .
$$

This will imply that each contracts $x_{i j}$ breaks even:

$$
E_{\alpha_{n}}\left[\mu \cdot x \mid x_{i j}\right]=x_{i j} \mu_{i+1} \cdot \frac{\alpha_{n}\left(\mu_{i}, x_{i j}\right)}{\alpha_{n}\left(\mu_{i}, x_{i j}\right)+\eta_{n}\left(x_{i, j}\right)}+0=x_{i j} \mu_{i+1} \cdot \frac{P_{i} \cdot \frac{1}{n^{2}}}{P_{i} \cdot \frac{1}{n^{2}}+\eta_{n}\left(x_{i, j}\right)}=g_{i}\left(x_{i j}\right)=p\left(x_{i j}\right)
$$




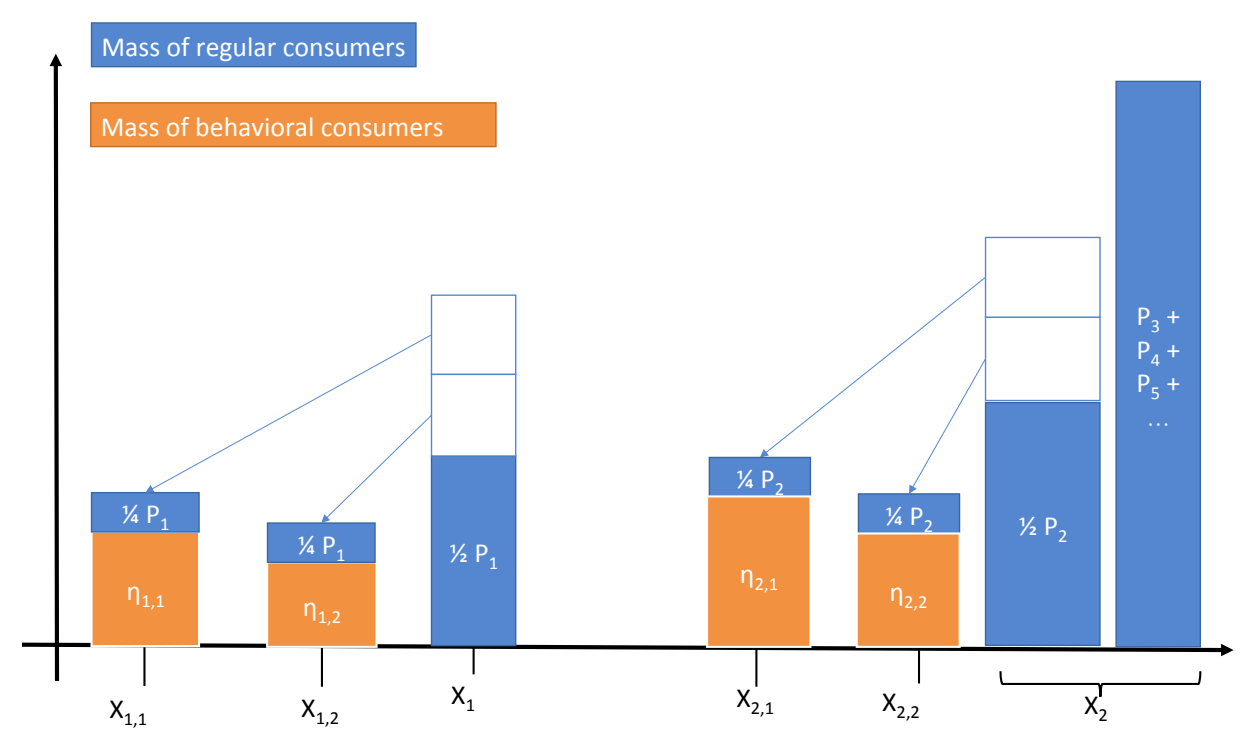

Figure 6: Illustration of $\alpha_{n}$ used in Section F. Specifically, the figure illustrates the second perturbation, $\alpha_{3}$.

Moreover, since $x_{i-1} \leq x_{i j} \leq x_{i}$, we also have

$$
\frac{g_{i}\left(x_{i-1}\right)}{x_{i} \mu_{i}} \leq \frac{P_{i} \cdot \frac{1}{n^{2}}}{P_{i} \cdot \frac{1}{n^{2}}+\eta_{n}\left(x_{i j}\right)}
$$

and therefore, as $n \rightarrow \infty$, we have $\sup _{x_{i j}} \eta_{n}\left(x_{i j}\right) \rightarrow 0$.

We also assume that, in economy $n$, there are no behavioral types purchasing contracts $x_{i}$ for $i \leq n-1$ :

$$
\eta_{n}\left(x_{i}\right)=0, i=1, \ldots, n-1
$$

Regarding the top contract $x_{n}$, the mass of behavioral types $\eta_{n}\left(x_{n}\right)$ is defined such that

$$
\mu_{n}=\frac{p\left(x_{n}\right)}{x_{n}}=E_{\alpha_{n}}\left[\mu \mid x_{n}\right]=\frac{\mu_{n} P_{n} \frac{n-1}{n}+\sum_{j>n} \mu_{j} P_{j}}{P_{n} \frac{n-1}{n}+\sum_{j>n} P_{j}+\eta_{n}\left(x_{n}\right)}
$$

i.e., $\eta_{n}\left(x_{n}\right)$ is chosen such that although the riskiest agents all choose the top contract, its

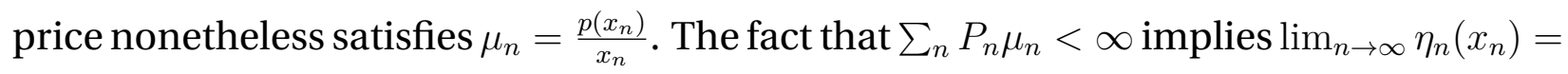
0 .

In this way, for each $i$, the break even condition $E_{\alpha_{n}}\left[\mu x \mid x_{i}\right]=p_{n}\left(x_{i}\right)=p\left(x_{i}\right)$ holds for each $i=1, \ldots, n$ in $\left(p_{n}, \alpha_{n}\right)$; indeed, for each $i=1, \ldots, n-1$, only types $\mu_{i}$ purchase $x_{i}$, while for $i=n$ this results from our definition of $p_{n}\left(x_{n}\right)=p\left(x_{n}\right)$ and by (24). 


\section{I.2 Convergence to equilibrium of unbounded economy}

We now prove that the equilibria described above for each truncated economy $\mathcal{E}^{n}$ converge to the equilibrium of $\mathcal{E}$.

Proof. We claim that the sequence $\left(p_{n}, \alpha_{n}\right)$ demonstrates that $(p, \alpha)$ is an equilibrium. , $p_{n} \equiv p$ on $\bar{X}^{n}$ and $\bar{X}^{n} \rightarrow X^{\prime}=\left[x_{0}, 1\right)$ in the sense of Haussdorf.

Moreover, $\alpha_{n} \rightarrow \alpha$ weakly: Notice $\alpha_{n}$ is concentrated on the set of types $\left\{\left(\mu_{k}, \nu_{k}\right)\right\}_{k \in \mathbb{N}}$ and the behavioral types, with $\alpha_{n}\left(\mu_{k}, \nu_{k}\right)=\alpha\left(\mu_{k}, \nu_{k}\right)=P_{k}$. Define $\mathbb{I}_{k, m}=1\{k=m\}$ be an indicator function. Then,

$$
\alpha_{n}\left(\mu_{k}, x_{m}\right)=\mathbb{I}_{k, m} \cdot P_{m}\left[1-\frac{1}{n}\right] \rightarrow \mathbb{I}_{k, m} \cdot P_{m}=\alpha\left(\mu_{k}, x_{m}\right)
$$

and for each $m \in \mathbb{N}$,

$$
\alpha_{n}\left(\left\{x \in\left(x_{m-1}, x_{m}\right)\right\}\right)=P_{m} \frac{1}{n} \rightarrow 0=\alpha\left(\left\{x \in\left(x_{m-1}, x_{m}\right)\right\}\right) .
$$

Hence for each $\delta<1, \alpha_{n}(\cdot \mid\{x \leq \delta\}) \rightarrow \alpha(\cdot \mid\{x \leq \delta\})$ converges in total variation norm. This implies that $\alpha_{n} \rightarrow \alpha$ weakly.

Furthermore, $\alpha_{n}$-a.s. the original agents $\left\{\left(\mu_{n}, \nu_{n}\right)\right\}_{n=1}^{\infty}$ are utility maximizing: agents of type $i \leq n$ are utility maximizing since they either choose the same option $x_{i}$ in $\bar{X}^{n} \subseteq X=[0,1)$, at the same price $p_{n}\left(x_{i}\right)=p\left(x_{i}\right)$, as they do when they can choose any alternative in $X$, or they choose an alternative $x_{i, 1}, \ldots, x_{i, n}$ which delivers the same utility as $x_{i}$ at prices $p_{n} \equiv p$. Agents of type $k>n$ are utility maximizing since their willingness to pay for $x$ is higher than that of type $n$, who (weakly) prefers the contract $x_{n}=\max \left[\bar{X}^{n}\right]$ to any other alternative in $\bar{X}^{n}$ at prices $p_{n} \equiv p$. Therefore, each $\left(p_{n}, \alpha_{n}\right)$ is a weak equilibrium, so $(p, \alpha)$ is an equilibrium.

\section{J Weakening Assumption 7}

We remark that Proposition 8 would hold if Assumption 7 would be replaced with the following weaker assumption, although the proof would be somewhat more technical and lengthier.

Assumption 9. For each $\mu^{\prime} \in \operatorname{supp}\left(P_{\mu}\right)$ with $\mu^{\prime}>\underline{\mu}\left(:=\min \operatorname{supp}\left(P_{\mu}\right)\right)$, for each $0<x_{1}<$ $x_{2}<1$, and each $0<p_{1}<p_{2}$, there is $\varepsilon>0$ s.t. denoting $D=\left[x_{1}, x_{2}\right] \times\left[p_{1}, p_{2}\right]$ and $T=\left\{\theta \mid \mu^{\prime}-\varepsilon<\mu(\theta) \leq \mu^{\prime}\right\}$.

$$
\inf _{T} \min _{D}\left(w_{\theta}(x, p)-\mu_{\theta}\right)>0 \text { and } \inf _{T} \min _{D} \frac{\partial u_{\theta}}{\partial p}<0
$$


We know for each $x<1$ and $p \in \mathbb{R}, w_{\theta}(x, p)-\mu_{\theta}>0$. Hence, by continuity, for each $\theta \in \Theta$, each $0<x_{1}<x_{2}<1$, and each $0<p_{1}<p_{2}, \min _{D}\left(w_{\theta}(x, p)-\mu_{\theta}\right)>0$. However, we need this positivity to be uniform over all types whose riskiness $\mu$ is close to any given $\mu^{\prime}$. Similarly, we need the sensitivity to price to be bounded away from 0 in such a domain for all types with riskiness close enough to $\mu^{\prime}$.

\section{K Other Proofs}

\section{K.1 Completion of Proof of Proposition 1}

We formalize the idea given at the end of Section A.1: Let $\rho_{n}$ be a measure on $\Theta \backslash \Theta_{n} \times$ $X_{n} \subseteq \Theta \times X_{n}$ s.t.

$$
\rho_{n}\left((\theta, x) \in \Theta \backslash \Theta_{n} \times X_{n} \mid \forall y \in X_{n}, u\left(x, \theta, q_{n}(x)\right) \geq u\left(y, \theta, q_{n}(y)\right)\right)=P\left(\Theta \backslash \Theta_{n}\right)
$$

- i.e., $\rho_{n}$ is an allocation for those types not in $\Theta_{n}$ in which they a.s. maximize their utility in $X_{n}$ at prices $q_{n}(\cdot)$. By assumption,

$$
E_{\gamma_{n}+\rho_{n}}[c \mid x] \geq E_{\gamma_{n}}[c \mid x]=r_{n}(x), \forall x \in \bar{W}_{n}
$$

with equality if $r_{n}(x)<c_{0}$, i.e., the types in $\Theta$ which not in $\Theta_{n}$ only increase costs. Note that $\gamma_{n}+\rho_{n}$ may not be normalized; throughout this proof, when $\sigma$ is a non-normalized measure, $E_{\sigma}[f]=\frac{1}{\sigma(\Theta)} \int_{\Theta} f d \sigma$. Define $\pi_{n}(x) \geq 0$ s.t.

$$
\frac{\gamma_{n}(x)+\rho_{n}(x)}{\gamma_{n}(x)+\rho_{n}(x)+\pi_{n}(x)} E_{\gamma_{n}+\rho_{n}}[c \mid x]=E_{\gamma_{n}}[c \mid x]=r_{n}(x), \forall x \in \bar{W}_{n}
$$

By assumption $\gamma_{n}(x)>0$ for all $x \in \bar{W}_{n}$ hence this is well defined. Hence, since cost of behavioral types is 0 ,

$$
E_{\rho_{n}+\gamma_{n}+\pi_{n}}[c \mid x]=E_{\gamma_{n}}[c \mid x]=r_{n}(x), \forall \mathbf{x} \in \bar{W}_{n}
$$

Furthermore, $\pi_{n}(x)=0$ if $x \in \bar{W}_{n}$ and $r_{n}(x)<c_{0}$, since no types in $\Theta \backslash \Theta_{n}$ purchases in the domain $r_{n}<c_{0}$, i.e., $\rho_{n}\left(\left\{(\theta, x) \mid r_{n}(x)<c_{0}\right\}\right)=0$. We contend that $\pi_{n}\left(\bar{W}_{n}\right) \rightarrow 0$, as required of behavioral types. Indeed, by definition,

$$
\left[\rho_{n}(x)+\gamma_{n}(x)\right] \cdot E_{\rho_{n}+\gamma_{n}}[c \mid x]=\gamma_{n}(x) \cdot E_{\gamma_{n}}[c \mid x]+\left[\pi_{n}(x)+\rho_{n}(x)\right] \cdot r_{n}(x), \forall x \in \bar{W}_{n}
$$


Therefore,

$\int c \cdot d\left(\gamma_{n}+\rho_{n}\right)=\int c \cdot d \gamma_{n}+\sum_{x \in \bar{W}_{n}}\left[\pi_{n}(x)+\rho_{n}(x)\right] \cdot r_{n}(x)=\int c \cdot d \gamma_{n}+\int r_{n} \cdot d \rho_{n}+\int r_{n} d \pi_{n}$

i.e.,

$$
c_{0} \cdot \pi_{n}\left(\bar{W}_{n}\right) \leq \int r_{n} d \pi_{n}=\int\left(c-r_{n}\right) \cdot d \rho_{n} \rightarrow 0
$$

where the second term vanishes due to our integrability requirement, and since $\rho_{n}$ is supported on $\Theta \backslash \Theta_{n}$. Hence, denoting $\delta_{n}=\gamma_{n}+\rho_{n}+\pi_{n}$ (where $\pi_{n}$ naturally induces a measure on the diagonal of $\left.\bar{W}_{n} \times \bar{W}_{n} \subseteq\left(\Theta \cup \bar{W}_{n}\right) \times \bar{W}_{n}\right)$ shows that the sequences of subspace $\left(\bar{W}_{n}\right)_{n \in \mathbb{N}}$, behavioral types $\left(\pi_{n}\right)_{n \in \mathbb{N}}$, and weak equilibrium $\left(r_{n}, \delta_{n}\right)_{n \in \mathbb{N}}$ witness $(p, \alpha)$ being an equilibrium.

\section{K.2 Completion of Proof of Proposition 4}

We complete the proof of Proposition 4 which began in Section F.

Proof. Let $f:[0,1] \times \mathbb{R}_{+}$be any continuous and bounded function. Let $P_{n}$ be the mass of the $n$-th type, and $Q_{j}^{n}=\frac{P_{j}}{\sum_{j \leq n} P_{j}}$ be the conditional mass in the $n$-th economy. The conditional distributions on types converges in norm to the distribution on the infinite type space. $f$ is continuous, so for each $k, f\left(x_{k}^{n}\right) \rightarrow f\left(x_{k}\right)$. Hence, by the bounded convergence theorem,

$$
\int_{\Theta \times[0,1]} f d \alpha^{n}=\sum_{k=1}^{n} f\left(x_{k}^{n}\right) \cdot Q_{k} \rightarrow \sum_{k=1}^{\infty} f\left(x_{k}\right) \cdot P_{k}=\int_{\Theta \times[0,1]} f d \alpha
$$

and hence $\alpha^{n} \rightarrow \alpha$ weakly.

To show $p^{n} \rightarrow p$ uniformly on compact subsets of $[0,1)$, it suffices to show that for each interval of the form $I_{k}=\left[x_{k}, x_{k+1}\right], p^{n} \rightarrow p$ uniformly in $I_{k}$. The $\left(p^{n}\right)$ are uniformly Lipschitz in $I^{k}$, with Lipschitz constant $L=\mu_{k+1}+\nu_{k+1} \cdot \sup _{[0,1]} g^{\prime}$, as $x_{k+1}^{n} \geq x_{k+1}$. Furthermore, since $g_{k+1}^{n}$ (as the indifference curve of type $k+1$ through $x_{k+1}^{n}$ ) converges point-wise to $g_{k+1}$ (as the indifference curve of type $k$ through $x_{k}^{n}$ ), $p$ coincides with $g_{k+1}$ in $I_{k}$, and $p^{n}$ coincides with $g_{k+1}^{n}$ in $\left[x_{k}^{n}, x_{k+1}^{n}\right]$, we have $p^{n} \rightarrow p$ point-wise. Then, pointwise convergence and uniform Lipschitz implies uniform convergence.

\section{K.3 Proof of Proposition 6}

Before describing the proof, we present a useful generalization of the Arzela-Ascoli theorem. This generalization is found, for instance, in Kelley [2017, Thm 17, Ch 7]. The key generalization in this version is the requirement that $X$ be only locally compact (rather 
than compact), and the requirement that the functions $f$ to be point-wise bounded (rather than uniformly bounded).

Theorem 3. Let $X$ be a locally compact metric space. Given a sequence $\left(f_{n}\right)_{n=1}^{\infty}$ of realvalued functions on $X$, equicontinuous and point-wise bounded, there is a continuous function $f: X \rightarrow \mathbb{R}$ and a subsequence of $\left(f_{n}\right)_{n=1}^{\infty}$ converging to $f$ uniformly on compact sets.

We now prove Proposition 6:

Proof. The existence of the limit function $p(\cdot)$ with the required first property of $p(\cdot)$ in Proposition 1 follows from Theorem 3. Furthermore, $\left(\alpha_{n}\right)_{n=1}^{\infty}$ (or any of its subsequences) is tight (since $\alpha_{n}\left(\Theta_{k} \times \bar{X}\right)=P\left(\Theta_{k}\right)$ for all $n, k \in \mathbb{N}$ ) and hence it w.l.o.g. (passing to a subsequence) converges weakly to some measure $\alpha$ on $\Theta \times \bar{X}$.

We now need to show that $\alpha(\Theta \times(\bar{X} \backslash X))=0$. Suppose not, set $B:=\alpha(\Theta \times \bar{X} \backslash X)>0$. Fix some $y_{0} \in X$, and fix some $D>\sup _{n} p_{n}\left(y_{o}\right)$. (By assumption, such $D<\infty$ exists.) We note that, by assumption, for each $\theta \in \Theta$, and each alternative $y \in X$, there is $q \geq 0$ s.t. $u\left(y_{0}, \theta, D\right)>u(y, \theta, q)$; by possibly decreasing $D$ slightly, the continuity of utility in $\bar{X} \times$ $\Theta \times \mathbb{R}_{+}$shows that this statement is true for all $y \in \bar{X}$; and finally a standard continuity argument shows that $q$ may be chosen independent of $y \in X$ (only dependent on $\theta$ ); i.e., $\cap_{M>0}\left\{\theta \mid \exists y \in \bar{X}\right.$ s.t $\left.u\left(y_{0}, \theta, D\right) \leq u(y, \theta, M)\right\}=\emptyset$. Fix $M$ s.t.

$$
P\left(\left\{\theta \mid \exists y \in \bar{X} \text { s.t } u\left(y_{0}, \theta, D\right) \leq u(y, \theta, M)\right\}\right)<\frac{1}{2} B
$$

By assumption, there is a neighborhood $V$ of $\bar{X} \backslash X$ such that for all $n$ large enough and all $y \in V, p_{n}(y)>M$. Therefore, $\alpha_{n}(\Theta \times V) \leq \frac{1}{2} B$ for all $n$ large enough. By Portmanteau theorem, however, since $V$ is open

$$
\frac{1}{2} B \geq \liminf \alpha_{n}(\Theta \times V) \geq \alpha(\Theta \times V) \geq \alpha(\Theta \times \bar{X} \backslash X)=B>0,
$$

a contradiction.

\section{K.4 Proof of Proposition 7}

Proof. That Assumption 6 implies single crossing is immediate. Conversely, suppose single crossing holds. First, fix types $\theta_{1}, \theta_{2} \in \Theta$ with $\mu\left(\theta_{2}\right) \geq \mu\left(\theta_{1}\right)$ but by way of contradiction, there were some $x_{0}, p_{0} \in[0,1] \times \mathbb{R}_{+}$at which $w_{\theta_{2}}\left(x_{0}, p_{0}\right)<w_{\theta_{1}}\left(x_{0}, p_{0}\right)$. We may assume $\mu\left(\theta_{2}\right)>\mu\left(\theta_{1}\right)$, as we may alter slightly $\theta_{2}, \theta_{1}$ (by our regularity properties). Let $g:\left[x_{0}, 1\right] \rightarrow \mathbb{R}_{+}$be s.t. $u_{\theta_{2}}\left(x_{0}, p_{0}\right)=u_{\theta_{2}}(x, g(x))$ for all $x \in\left[x_{0}, 1\right]$, and for each $x \in\left[x_{0}, 1\right]$, let $h(x)$ denote the price $p$ s.t. $u_{\theta_{1}}(1, p)=u_{\theta_{1}}(x, g(x))$; both $g, h$ are welldefined and continuous by the implicit function theorem. Since $w_{\theta_{2}}\left(x_{0}, p_{0}\right)<w_{\theta_{1}}\left(x_{0}, p_{0}\right)$, 
$u_{\theta_{1}}\left(x_{0}, p_{0}\right)<u_{\theta_{1}}(x, g(x))$ in a right-neighborhood of $x_{0}$. By single-crossing and continuity of $h, h>g(1)$. However, $w_{\theta_{1}} \approx \mu_{1}<w_{\theta_{2}} \approx \mu_{2}$ in a neighborhood of $(1, g(1)), h<g(1)$ in a punctured neighborhood of 1 , a contradiction. Conversely, if for some $\theta_{1}, \theta_{2} \in \Theta$, $x_{0}, p_{0} \in[0,1] \times \mathbb{R}_{+}$we had $w_{\theta_{2}}\left(x_{0}, p_{0}\right) \geq w_{\theta_{1}}\left(x_{0}, p_{0}\right)$ but $\mu\left(\theta_{2}\right)<\mu\left(\theta_{1}\right)$, we could (again, by the regularity properties) alter slightly $\theta_{2}, \theta_{1}$ so that $w_{\theta_{2}}\left(x_{0}, p_{0}\right)>w_{\theta_{1}}\left(x_{0}, p_{0}\right)$, and we would get a contradiction by what we've already shown.

\section{K.5 Proof of Lemma 8}

Proof. First we show that for each $\varepsilon>0$ for large enough $n$, under $\alpha_{n}$ a positive measure of types with riskiness $\geq \mu^{*}$ purchase in $I_{\varepsilon}:=\left(x^{*}-\varepsilon, x^{*}+\varepsilon\right)$. If there were such an $\varepsilon>0$ for which this did not hold, then for all $n$ large enough, $p^{n}(x) \leq \mu_{*} \cdot x$ for $x \in I_{\varepsilon}$; and hence $p(x) \leq \mu_{*} \cdot x$ for $x \in I_{\varepsilon}$, leading to a contradiction as in Lemma 6. Now, fix $\delta>0$. Any type with riskiness $\mu \geq \mu^{*}+\delta$ strictly prefers $\left(\sigma\left(\mu^{*}\right), p\left(\sigma\left(\mu^{*}\right)\right)\right.$ to $\left(x^{*}, p\left(x^{*}\right)\right)$, and hence by Proposition 12 for $n$ large enough will not purchase in $I_{\varepsilon}$ under $\alpha_{n}$; hence, for $n$ large enough, we can find a $\alpha_{n}$-positive mass of types with riskiness in $\left[\mu^{*}, \mu^{*}+\delta\right)$ purchasing in $I_{\varepsilon}$.

\section{K.6 Proposition Used For Section E}

Proposition 15. Suppose $P_{\mu}$, conditional on some interval $I=(\underline{\mu}, \bar{\mu})$, has full support with a.e. strictly positive density w.r.t. the Lebesgue measure, and $(p, \alpha)$ is an equilibrium with associated coverage function $\sigma(\mu)$. Suppose $\sigma(\underline{\mu})>0$ (i.e., a.e. type with riskiness in I purchases positive coverage). Then, denoting $J=(\sigma(\underline{\mu}), \sigma(\bar{\mu}))$ and letting $\alpha_{J}$ be the projection of $\alpha$ to alternatives and conditional on $J, \alpha_{J}$ is equivalent to the Lebesgue measure on $J$, i.e., $\alpha_{J}$ and the Lebesgue measure on $J$ are absolutely continuous w.r.t. each other. In other words, $\alpha_{J}$ and the Lebesgue measure on J have the same null sets or, equivalently, $\alpha_{J}$ has Lebesgue-a.e. positive density.

Proof. Note that $\sigma: I \rightarrow J$ is a strictly increasing bijection (and in particular, continuous), and $\alpha_{J}=P_{\mu}(\cdot \mid J) \circ \sigma^{-1}$. Hence, it suffices to show that both $\sigma$ and $\tau=\sigma^{-1}: J \rightarrow I$ are locally Lipschitz in the interior of $I, J$ respectively. By Theorem 2, $p$ is $L$-Lipschitz in $J$ for some $L>0$. Now, $p(\sigma(\mu))=\mu \cdot \sigma(\mu), P_{\mu}-a . s$. Since $\sigma$ is continuous and $P_{\mu}$ has full support in $I$, this implies $p(\sigma(\mu))=\mu \cdot \sigma(\mu), \forall \mu \in I$ and hence $\tau(x)=\frac{p(x)}{x}, \forall x \in J$.

Since $p$ is Lipschitz in $J$ and $\sigma(\underline{\mu})>0, \tau$ is Lipschitz. Observe that for fixed $z$, the mapping $w_{\tau(x)}(z)=\tau(x)+g^{\prime}(z) \cdot \nu(\tau(x))$ is well-defined $\alpha_{J}$ a.e.. Note that $\operatorname{supp}\left(\alpha_{J}\right)=J$, as $\sigma$ is continuous. So, for $\alpha_{J}$ a.e. every $x_{1} \in J$ and every $x_{2} \in J$,

$$
\frac{p\left(x_{2}\right)-p\left(x_{1}\right)}{x_{2}-x_{1}} \geq \int_{x_{1}}^{x_{2}} w_{\tau\left(x_{1}\right)}(x) d x
$$


Full Insurance in Support of Equilibrium

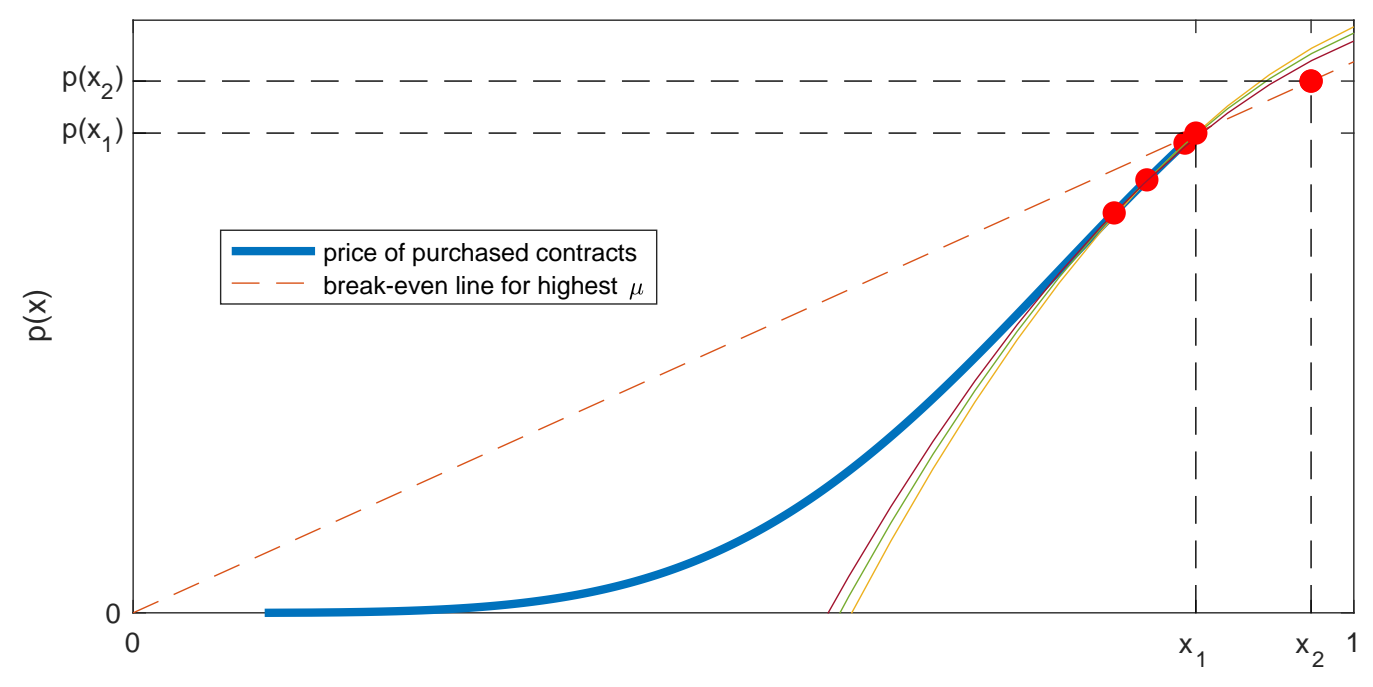

Figure 7: We consider, by way of contradiction, a hypothetical equilibrium where the supremum of contracts purchased is $x_{1}<1$. This implies, we show, that $\mu$ must be bounded by some $\bar{\mu}$, so the price of contracts $x>x_{1}$ is $p(x)=\bar{\mu} x$. Consider any $x_{2} \in$ $\left(x_{1}, 1\right)$. We then show that those agents who purchase $x$ close to $x_{1}$ would prefer $x_{2}$ (at the price $\left.p\left(x_{2}\right)=\bar{\mu} x_{2}\right)$, a contradiction.

and hence $p^{\prime}(x) \geq w_{\tau(x)}$ Lebesgue a.e.. Therefore, Lebesgue-a.s.,

$$
\tau^{\prime}(x)=\frac{1}{x}\left[p^{\prime}(x)-\frac{p(x)}{x}\right] \geq \frac{1}{x}\left[w_{\tau(x)}(x)-\tau(x)\right]=\frac{1}{x} g^{\prime}(x) \cdot \nu(\tau(x)) \geq g^{\prime}(x) \cdot \nu(\underline{\mu})
$$

Hence, in each sub-interval of $J$ which is bounded away from full insurance, $\tau^{\prime}>0$ is bounded away from 0 and hence $\sigma=\tau^{-1}$ is locally Lipschitz in the interior of $\tau(J)$.

\section{K.7 Proof of Proposition 12}

Proof. Suppose $u_{\mu_{0}}\left(p_{0}, x_{0}\right)>u_{\mu_{0}}\left(q_{0}, y_{0}\right)$. Suppose by way of contradiction there are sequences $\mu_{n} \rightarrow \mu_{0},\left(p_{n}, x_{n}\right) \rightarrow\left(p_{0}, x_{0}\right),\left(q_{n}, y_{n}\right) \rightarrow\left(q_{0}, y_{0}\right)$ s.t. $\mu_{n}$ weakly prefers $\left(q_{n}, y_{n}\right)$ over $\left(p_{n}, x_{n}\right)$. Let $\theta_{n} \in \Omega$ s.t. $\mu\left(\theta_{n}\right)=\mu_{n}$; since by Assumption $7\left\{\theta \mid \mu(\theta) \leq \mu_{0}+1\right\}$ is compact, w.l.o.g. and by passing to a subsequence, we may assume $\theta_{n} \rightarrow \theta_{0}$ for some $\theta_{0} \in \Theta$. Since $\mu$ is continuous, $\mu\left(\theta_{0}\right)=\mu_{0}$. Since $u\left(\theta_{n}, x_{n}, p_{n}\right) \leq u\left(\theta_{n}, y_{n}, q_{n}\right)$, taking the limit given by the continuity of utility implies $u\left(\theta_{0}, x_{0}, p_{0}\right) \leq u\left(\theta_{0}, y_{0}, q_{0}\right)$, a contradiction.

\section{K.8 Figure Complementing Lemma 7}

See Figure 7. 


\section{K.9 Completing Proof of Lemma 10}

Proof. We need to show the second and third inequalities. To show the third inequality, notice that for each $x_{1} \in[0,1]$, utility maximization implies

$$
\alpha\left(\left\{u(\theta, p(x), x) \geq u\left(\theta, p\left(x_{1}\right), x_{1}\right)\right\}\right)=1
$$

In particular, by the definition of $\psi^{-}$, there is a sequence $y_{n} \rightarrow x_{2}$ in $\operatorname{supp}\left(\alpha_{X}\right)$ and types $\left(\theta_{n}\right)$ with $\mu\left(\theta_{n}\right) \rightarrow \psi^{-}\left(x_{2}\right)$ (if $x_{2}$ is an atom of $\alpha_{X}$, take $\left.y_{n} \equiv x_{2}\right)$, such that for all $n$, $u\left(\theta_{n}, p\left(y_{n}\right), y_{n}\right) \geq u\left(\theta_{n}, p_{1}, x_{1}\right)$. Recall that $\bar{\rho}_{\bar{\delta}}(\cdot)$ is monotonically increasing and rightcontinuous. Hence also $\left(\bar{\rho}_{\bar{\delta}}\left(\theta_{n}\right)\right)$ satisfies $\lim \sup _{n \rightarrow \infty} \bar{\nu}_{\bar{\delta}}\left(\theta_{n}\right) \leq \bar{\rho}_{\bar{\delta}}\left(\psi^{-}\left(x_{2}\right)\right)$. An application of Lemma 9, the fact that $\liminf _{y \rightarrow x_{2}} \psi^{-}(y)=\psi^{-}\left(x_{2}\right)$ if $x_{2}$ is not an atom of $\alpha_{X}$, the rightcontinuity of $\bar{\rho}_{\bar{\delta}}$ and the continuity of $p$ on $\operatorname{supp}\left(\alpha_{X}\right)$ completes the proof. The second inequality follows similarly, by using the fact that that $\underline{\nu}_{\bar{\delta}}\left(\theta_{n}\right) \geq 0$.

\section{K.10 Proof of Lemma 11}

Proof. Since $p(\cdot)$ is part of an equilibrium, there are finite subsets $\bar{X}^{n} \subseteq X$, prices $p^{n}$ : $\bar{X}^{n} \rightarrow \mathbb{R}_{+}$and associated distributions $\alpha^{n}$ on $\left(\Theta \cup \bar{X}^{n}\right) \times \bar{X}^{n}$ as described in Section 2 which witness that $(p, \alpha)$ is an equilibrium. Let $y^{n} \rightarrow x_{1}$ and $z^{n} \rightarrow x_{2}$ with $y^{n}, z^{n} \in \bar{X}^{n}$. Since each $\left(p_{n}, \alpha_{n}\right)$ is a weak equilibrium whose projection to $X$ is finitely supported, it follows from Lemma 10 that, at each $n$,

$$
\frac{p_{n}\left(y_{n}\right)}{y_{n}} \leq \frac{p_{n}\left(z_{n}\right)-p\left(y_{n}\right)}{z_{n}-y_{n}} \leq \frac{p_{n}\left(z_{n}\right)}{z_{n}}+\left(1-\frac{y_{n}+z_{n}}{2}\right) \bar{\rho}_{\bar{\delta}}\left(\frac{p\left(z_{n}\right)}{z_{n}}\right)
$$

Taking $n \rightarrow \infty$, and recalling that $\bar{\rho}_{\bar{\delta}}$ is monotonically increasing and right-continuous, completes the proof.

\section{K.11 Proof for Section C.6 for Continuous Case}

Assume $P$ is continuous, but assume by way of contradiction that for some $\mu_{*}>\underline{\mu}=$ $\inf \operatorname{supp}\left(P_{\mu}\right), \sigma\left(\mu_{*}\right)=0$, and in particular 0 is an atom of $\alpha_{X}$, the projection of $\alpha$ to alternatives.

First we contend $\operatorname{supp}\left(\alpha_{X}\right)=[0,1]$; by the previous properties, $\operatorname{supp}\left(\alpha_{X}\right)=\{0\} \cup[\underline{x}, 1]$ for some $\underline{x} \geq 0$; if $\underline{x}>0$, an imitation of the argument used to prove the upper-envelope property of price would give a contradiction, as the indifference curve of any type purchasing near $\underline{x}$ would lie below $(0,0)$. Denote $\tau(x)=\frac{p(x)}{x}$. Following an argument similar to the proof of Proposition 13, with appropriate modifications, it follows that for 
Lebesgue a.e. $x \in(0, \delta)$ for $1>\delta>0$,

$p^{\prime}(x)=w_{\tau(x)}(x, p(x))=\tau(x)+w_{\tau(x)}(x, p(x))-\tau(x) \geq \frac{p(x)}{x}+\inf _{x \in J}\left(w_{\tau(x)}(x, p(x))-\tau(x)\right)=\frac{p(x)}{x}+W$

for some $W>0$. (This equation encompasses that the insurance surplus is bounded away from 0 in the interval $(0, \delta)$ uniformly over all types with riskiness at least $\mu_{*}$.) Hence

$$
\left(\frac{p(x)}{x}\right)^{\prime}=\frac{p^{\prime}(x)}{x}-\frac{p(x)}{x^{2}} \geq \frac{W}{x} \longrightarrow \frac{p(\delta)}{\delta} \geq \frac{p(\delta)}{\delta}-\liminf _{y \rightarrow 0} \frac{p(y)}{y} \geq W \cdot \lim _{y \rightarrow 0} \ln \left(\frac{\delta}{y}\right)=\infty
$$

